\title{
FRACTIONAL POWERS AND INTERPOLATION THEORY FOR MULTIVALUED LINEAR OPERATORS AND APPLICATIONS TO DEGENERATE DIFFERENTIAL EQUATIONS
}

\author{
By
}

\author{
Alberto Favaron and Angelo FavinI
}

\begin{abstract}
We provide intermediate properties for the domains of the fractional powers of an abstract multivalued linear operator $A$ of weak parabolic type. In particular, our results exhibit the special role played by the linear subspace $A 0$, which reduces to $\{0\}$ if and only if $A$ is single-valued. The behaviour of the singular semigroup generated by $A$ with respect to the domains of the fractional powers is then studied, and applications of this behaviour to questions of maximal time and space regularity for abstract multivalued evolution equations are given. As a concrete case we consider a class of degenerate partial differential evolution equations which may be rewritten in a multivalued evolution form.
\end{abstract}

\section{Introduction}

The aim of this paper is to establish some intermediate properties for the domains of the fractional powers of abstract multivalued linear operators. The class of operators we shall deal with consists of those multivalued linear operators $A$ from a complex Banach space $X$ to itself, which have a single-valued resolvent satisfying the following estimate:

$$
\left\|(\lambda I-A)^{-1} x\right\|_{X} \leq C(|\lambda|+1)^{-\beta}\|x\|_{X}, \quad \forall \lambda \in \Sigma_{\alpha}, \forall x \in X .
$$

Here, $I$ is the identity operator, $C$ is a positive constant, $\beta \in(0,1]$ and $\Sigma_{\alpha}$ is the complex region $\left\{z \in \mathbf{C}: \Re \mathrm{e} z \geq-c(|\Im \mathrm{m} z|+1)^{\alpha}\right\}, c>0, \alpha \in[\beta, 1]$.

Mathematics Subject Classification (2000): Primary 46B70, 47A06, 47B99; Secondary 35K65, 44A45, $47 \mathrm{D} 06$.

Keywords: Fractional powers; interpolation theory; multivalued linear operators; singular semigroups; multivalued evolution equations; degenerate differential equations.

Received May 30, 2011. 
To elucidate the motivation of our research, we make a brief digression in the ambit of single-valued operators. To this purpose, recall that if $A$ is a single-valued densely defined linear operator satisfying (1.1) with $\beta=1$, then the domains $\mathscr{D}\left((-A)^{\theta}\right)$ of its fractional powers $(-A)^{\theta}$ enjoy the following intermediate property (cf. [24], [25] and [35]):

$$
(X, \mathscr{D}(A))_{\Re \mathrm{e} \theta, 1} \hookrightarrow \mathscr{D}\left((-A)^{\theta}\right) \hookrightarrow(X, \mathscr{D}(A))_{\Re \mathrm{e} \theta, \infty}, \quad \Re \mathrm{e} \theta \in(0,1),
$$

$(X, \mathscr{D}(A))_{\gamma, p}, \gamma \in(0,1), p \in[1, \infty]$, being real interpolation spaces between $X$ and the domain $\mathscr{D}(A)$ of $A$. Relation (1.2) is decisive in a lot of abstract results whose proofs employ interpolation theory's techniques. For instance, it is the starting point in the abstract theory of semilinear parabolic equations (cf. [20] and [27]). On the contrary, if $(\alpha, \beta) \neq(1,1)$ and/or $\mathscr{D}(A)$ is not dense, it is not known whether embedding (1.2), or at least a similar one, continues to hold. Since estimate (1.1) with $\beta \in(0,1)$ occurs in many concrete cases (cf. [29], [32], [36], [37] and [38]), it is clear how much the question of generalizing (1.2) necessitates to be answered, even if only for the single-valued case.

Although a single-valued approach would certainly have been easier, we have preferred to treat the problem in the more general context of multivalued linear operators. This choice depends on two reasons. The first is that any single-valued linear operator $A$ is by itself a trivial multivalued one, in which the linear subspace $A 0$ reduces to $\{0\}$. Consequently, the generalization of (1.2) for the single-valued case will be obtained simply by taking $A 0=\{0\}$ in the result that we are going to describe. The second reason is that the applications we have in mind concern degenerate differential equations of the type considered in [8]-[13], [28] and [40]. The standard procedure for solving such equations is to rewrite them in a non degenerate multivalued form, where the basic multivalued linear operator $A$ satisfies $(1.1)$ and the case $\beta \in(0,1)$ appears in many concrete circumstances. As shown in [12], the belonging of some data to $\mathscr{D}\left((-A)^{\theta}\right)$ for opportune values of $\theta$ is in general sufficient to get existence, uniqueness and regularity results for this kind of equations. On the other hand, when dealing with questions of maximal regularity in both time and space, the absence of any known intermediate property for the domains of the fractional powers of multivalued linear operators yields to assume that the data belong to some unnatural intersections as those in [8]; intersections which could be easily characterized if we were in possess of a relation of type (1.2).

To highlight the novelty of our results we remind that, contrarily to the single-valued case with $\beta=1$ for which the theory goes back to [4], [19] and [23]-[25], the study of fractional powers of multivalued linear operators satisfying 
(1.1) is still a quite unexplored field. To our knowledge, fractional powers of multivalued linear operators were first introduced in [1] for the case $\beta=1$, and subsequently in [11] and [12] for the general case. We quote also [32], but only for the single-valued sub-case with $\alpha=1$. However, in none of these papers intermediate properties of type (1.2) were investigated. In the monograph [6], fractional powers are not even mentioned.

We now provide the detailed contents of this paper. Section 2 contains all the needed preliminary material of the theory of multivalued linear operators. At first, we introduce the basic concepts of inversion, sum, product, extensions and sections of multivalued linear operators. For a multivalued linear operator $A$ from $X$ to itself, we then pay particular attention to its resolvent set $\rho(A)$ and to the bounded section $z(z I-A)^{-1}-I$ of $A(z I-A)^{-1}, z \in \rho(A)$. It is here that the linear subspace $A 0$ begins to reveal its crucial role (cf. Lemma 2.1). Finally, to those multivalued linear operators $A$ satisfying (1.1), we associate the corresponding infinitely differentiable semigroup $\left\{\mathrm{e}^{t A}\right\}_{t \geq 0}$ on $X$.

In Section 3 we introduce the fractional powers $(-A)^{ \pm \theta}, \Re \mathrm{e} \theta>1-\beta$, of a multivalued linear operator $A$ satisfying (1.1). We first define the negative fractional powers $(-A)^{-\theta}, \Re \mathrm{e} \theta>1-\beta$, by explicit complex integrals converging in the $\mathscr{L}(X)$-norm and we briefly recall their main properties. The positive fractional powers $(-A)^{\theta}, \Re \mathrm{e} \theta>1-\beta$, are then defined as the inverse of $(-A)^{-\theta}$. Therefore, since in the really multivalued case the negative fractional powers are not injective, the positive fractional powers turn out to be a class of multivalued linear operators. Some relations between the domains $\mathscr{D}\left((-A)^{\theta}\right)$ of $(-A)^{\theta}$ are provided for different values of $\Re \mathrm{e} \theta>1-\beta$, and the semigroup property is investigated. In Remarks 3.5-3.8 we compare the single- and the multivalued cases. In particular, we show that the method of the closed extension, used in [4] and [23] to define $(-A)^{\theta}, \Re \mathrm{e} \theta>0$, for single-valued densely defined linear operators satisfying (1.1) with $\beta=1$, fails in the really multivalued case. It is for this failure that in the multivalued case the definition of $(-A)^{\theta}$ as multivalued inverse of $(-A)^{-\theta}$ is the only possible, with the remarkable consequence that an explicit formula for $(-A)^{\theta}$ is not at our disposal anymore. As a result of this lack, it is not clear, not even in the case of $\beta=1$, how to define the purely imaginary powers $(-A)^{i t}, t \in \mathbf{R}$, of a really multivalued linear operator $A$. We conclude the section introducing the bounded operators $\left[(-A)^{\theta}\right]^{\circ} \mathrm{e}^{t A}, t>0$, $\Re$ e $\theta \geq 0$, and the relative estimates for their $\mathscr{L}(X)$-norm.

For a multivalued linear operator $A$ satisfying (1.1), in Section 4 we introduce the Banach spaces $(X, \mathscr{D}(A))_{\gamma, p}$ and $X_{A}^{\gamma, p}, \gamma \in(0,1), p \in[1, \infty]$. The main result of the section is Proposition 4.3, where we extend to $p \in[1, \infty]$ some 
embedding relations among such spaces shown in [12] only for $p=\infty$. From Proposition 4.3 it follows that, with the exception of $\beta=1$ when $(X, \mathscr{D}(A))_{\gamma, p}$ and $X_{A}^{\gamma, p}$ coincide with equivalent norms, the spaces $X_{A}^{\gamma, p}$ are intermediate between $X$ and $\mathscr{D}(A)$ only for $\gamma \in(0, \beta)$, whereas they may be smaller than $\mathscr{D}(A)$ for $\gamma \in[\beta, 1)$. Our interest on spaces $X_{A}^{\gamma, p}$ for values of $p$ other than $p=\infty$ is motivated by the key role that spaces $X_{A}^{\gamma, 1}$ play in the theory of fractional powers of single-valued linear operators satisfying (1.1) with $\beta=1$. Of course, in this case they coincide with the interpolation spaces $(X, \mathscr{D}(A))_{\gamma, 1}$, but their definition makes them suitable for the construction of a closable operator having domain $X_{A}^{\Re \mathrm{e} \theta, 1}, \Re \mathrm{e} \theta \in(0,1)$, and whose closure is just $(-A)^{\theta}$ (cf. Remark 4.1). Even though in the really multivalued case we can not proceed at the same way, in a sense that we clarify soon below the basic idea still remains valid. An interesting property of spaces $X_{A}^{\gamma, p}$ is that they have in common with $A 0$ the solely zero element of $X$ (cf. Remark 4.2). It thus suffices to investigate only a relation of type

$$
X_{A}^{\gamma_{1}, p_{1}} \hookrightarrow\{0\} \cup\left[\mathscr{D}\left((-A)^{\theta}\right) \backslash A 0\right] \hookrightarrow X_{A}^{\gamma_{2}, p_{2}},
$$

for opportune $\Re \mathrm{e} \theta, \gamma_{j}$ and $p_{j}, j=1,2$. Clearly, if $A$ is single-valued and $\beta=1$, then we should obtain $\gamma_{1}=\gamma_{2}=\Re \mathrm{e} \theta \in(0,1)$ and $\left(p_{1}, p_{2}\right)=(1, \infty)$.

As byproduct of Proposition 4.3, in Section 5 we characterize the regularity of $\left\{\mathrm{e}^{t A}\right\}_{t \geq 0}$ with respect to the space $Y_{\gamma}^{p} \in\left\{(X, \mathscr{D}(A))_{\gamma, p}, X_{A}^{\gamma, p}\right\}, \gamma \in(0,1), p \in[1, \infty]$. First, in Lemma 5.1 we prove that the uniform norm $\left\|\left[(-A)^{1}\right]^{\circ} \mathrm{e}^{t A}\right\|_{\mathscr{L}\left(Y_{\gamma}^{p} ; X\right)}$ may blow-up as $t$ goes to zero, but not faster than $t^{(\beta+\gamma-2) / \alpha}$. Then, in Proposition 5.2 we show that $\left\{\mathrm{e}^{t A}\right\}_{t \geq 0}$ is strongly continuous in the $X$-norm on the space $Y_{\gamma}^{p}$, $\gamma \in(1-\beta, 1)$. Finally, in Corollary 5.4 we derive that the map $t \rightarrow \mathrm{e}^{t A}$ is Hölder continuous from $[0, \infty)$ to $\mathscr{L}\left(Y_{\gamma}^{p} ; X\right), \gamma \in(2-\alpha-\beta, 1)$, with Hölder exponent $\sigma=(\alpha+\beta+\gamma-2) / \alpha$.

Section 6 contains the proof of (1.3). We first introduce the Banach spaces $\mathscr{D}_{A}(\gamma, p), \gamma \in(0,1), p \in[1, \infty]$, and in Proposition 6.3 we prove some embedding relations among the spaces $X_{A}^{\gamma, p},(X, \mathscr{D}(A))_{\gamma, p}$ and $\mathscr{D}_{A}(\gamma, p)$. In particular, we obtain $\{0\} \cup\left[\mathscr{D}_{A}(\gamma, \infty) \backslash A 0\right] \hookrightarrow X_{A}^{\sigma, \infty}$, where $\gamma \in(2-\alpha-\beta, 1)$ and $\sigma$ is as above. Since $\mathscr{D}\left((-A)^{\theta}\right) \hookrightarrow \mathscr{D}_{A}(\Re \mathrm{e} \theta, \infty), \Re \mathrm{e} \theta \in(2-\alpha-\beta, 1)$, this yields (cf. Theorem 6.9):

$$
\{0\} \cup\left[\mathscr{D}\left((-A)^{\theta}\right) \backslash A 0\right] \hookrightarrow X_{A}^{(\alpha+\beta+\Re \mathrm{e} \theta-2) / \alpha, \infty}, \quad \Re \mathrm{e} \theta \in(2-\alpha-\beta, 1) .
$$

In Theorem 6.6 we focus on the embedding on the left of (1.3). In a certain sense, we generalize to the multivalued case the idea of the method of the closed extension. More precisely, we define a single-valued linear operator with domain 
$X_{A}^{\Re \mathrm{e} \theta, 1}, \Re \mathrm{e} \theta \in(1-\beta, \beta)$, and we prove that it is a section of the restriction of $(-A)^{\theta}$ to $X_{A}^{\Re e \theta, 1}$. Due to the mentioned property of the spaces $X_{A}^{\gamma, p}$, this leads to

$$
X_{A}^{\Re \mathrm{e} \theta, 1} \hookrightarrow\{0\} \cup\left[\mathscr{D}\left((-A)^{\theta}\right) \backslash A 0\right], \quad \Re \mathrm{e} \theta \in(1-\beta, \beta) .
$$

Finally, provided that $\Re \mathrm{e} \theta \in(2-\alpha-\beta, \beta)$, in Theorem 6.10 we combine (1.4) and (1.5) and we get (1.3) with $\left(p_{1}, p_{2}\right)=(1, \infty), \gamma_{1}=\Re$ e $\theta$ and $\gamma_{2}=$ $(\alpha+\beta+\Re \mathrm{e} \theta-2) / \alpha$.

In Section 7 we investigate the behaviour of the bounded operators $\left[(-A)^{\theta}\right]^{\circ} \mathrm{e}^{t A}, t>0, \Re \mathrm{e} \theta \geq 0$, with respect to the domains $\mathscr{D}\left((-A)^{\gamma}\right), \Re \mathrm{e} \gamma>1-\beta$. In Proposition 7.3 we show that the uniform norm $\left\|\left([-A]^{\theta}\right)^{\circ} \mathrm{e}^{t A}\right\|_{\mathscr{L}\left(X ; \mathscr{D}\left((-A)^{\gamma}\right)\right)}$ may goes to infinity as $t$ goes to zero, but not faster than $t^{(\beta-\Re \mathrm{e} \gamma-\Re \mathrm{e} \theta-1) / \alpha}$. This enables us to characterize the time and space regularity of some basic operator functions which appear naturally in the study of multivalued evolution equations (cf. Lemmas 7.6, 7.7, 7.10 and 7.11).

Section 8 contains applications of our results to questions of maximal regularity for multivalued and degenerate evolution equations. First, for a multivalued linear operator $A$ satisfying (1.1) we consider the problem

$$
D_{t} u(t) \in A u(t)+f(t), \quad t \in(0, T], \quad u(0)=u_{0},
$$

and in Theorem 8.2 we show that it has a unique solution $u \in C^{1+\sigma}([0, T]$; $\mathscr{D}\left((-A)^{\gamma}\right)$, provided that $\sigma$ and $\Re$ e $\gamma$ are opportunely chosen and the data pair $\left(f, u_{0}\right)$ is regular enough. We then consider the class of degenerate evolution equations

$$
D_{t}(M v(t))=L v(t)+f(t), \quad t \in(0, T], \quad M v(0)=u_{0},
$$

where $M$ and $L$ are single-valued linear operators in $X$ such that $\mathscr{D}(L) \subseteq \mathscr{D}(M)$ and $M$ may have no bounded inverse. Hence, $M^{-1}$ being generally defined only as a multivalued linear operator, problem (1.7) is completely equivalent to (1.6) with $(u, A)=\left(M v, L M^{-1}\right)$. Of course, $A$ is wanted to satisfy (1.1), and this is done by requiring $\left\|M(\lambda M-L)^{-1}\right\|_{\mathscr{L}(X)} \leq C(|\lambda|+1)^{-\beta}, \lambda \in \Sigma_{\alpha}$. It thus follows from Theorem 8.2 that problem (1.7) has a unique solution $v$ such that $M v \in$ $C^{1+\sigma}\left([0, T] ; \mathscr{D}\left((-A)^{\gamma}\right)\right.$. Applications of this result are then given to two concrete situations. We conclude the section suggesting possible applications of our results to the semilinear and non autonomous versions of problem (1.6).

Finally, in Section 9, for a multivalued linear operator $A$ satisfying (1.1) and under an additional hypothesis on the ranges of $(-A)^{-\theta}$, $\Re \mathrm{e} \theta \in[\beta, 1] \subset(1-\beta, 1]$, $\beta>1 / 2$, we provide a possible definition of the fractional powers $(-A)^{ \pm \theta}$, $\Re$ e $\theta \in[0,1-\beta]$, as a class of multivalued linear operators. In particular, if $A$ is 
single-valued, densely defined and satisfies (1.1) with $\beta=1$, then we restore the definition of $(-A)^{i t}, t \in \mathbf{R}$, given in [24].

\section{Basic Notions on Multivalued Linear Operators}

Let $X$ be a complex Banach space endowed with norm $\|\cdot\|_{X}$ and let $2^{X}$ be the collection of all the subsets of $X$. For a number $\lambda \in \mathbf{C}$ and three subsets $F_{i}$ of $2^{X} \backslash \varnothing, i=1,2,3, \lambda F_{1}$ and $F_{2}+F_{3}$ denote the subsets of $X$ defined by $\left\{\lambda f_{1}: f_{1} \in F_{1}\right\}$ and $\left\{f_{2}+f_{3}: f_{i} \in F_{i}, i=2,3\right\}$, respectively. Then, a mapping $A$ from $X$ into $2^{X}$ is called a multivalued linear operator in $X$, shortened to $\mathrm{m}$. 1 . operator, if its domain $\mathscr{D}(A)=\{x \in X: A x \neq \varnothing\}$ is a linear subspace of $X$ and $A$ satisfies:

i) $A x+A y \subset A(x+y), \forall x, y \in \mathscr{D}(A)$;

ii) $\lambda A x \subset A(\lambda x), \forall \lambda \in \mathbf{C}, \forall x \in \mathscr{D}(A)$.

Given $U \in 2^{X}$ we write $A(U)=\bigcup_{u \in \mathscr{D}(A) \cap U} A u$ and the set $\mathscr{R}(A)=A(X)=$ $A(\mathscr{D}(A))$ is called the range of $A$. If $\mathscr{R}(A)=X$, then $A$ is said to be surjective. The subset $\mathscr{G}(A)$ of $X \times X$ defined by $\{(x, y): x \in \mathscr{D}(A), y \in A x\}$ is called the graph of $A$. If $U \in 2^{X}$ is such that $U \cap \mathscr{D}(A) \neq \varnothing$, then the restiriction $\left.A\right|_{U}$ of $A$ to $U$ is the m. 1. operator having domain $\mathscr{D}\left(\left.A\right|_{U}\right)=U \cap \mathscr{D}(A)$ and such that $\left(\left.A\right|_{U}\right) x=A x, x \in \mathscr{D}\left(\left.A\right|_{U}\right)$. The following properties of a m. 1 . operator $A$ are immediate consequences of its definition (cf. [12]):

iii) $A x+A y=A(x+y), \forall x, y \in \mathscr{D}(A)$;

iv) $\lambda A x=A(\lambda x), \forall \lambda \in \mathbf{C} \backslash\{0\}, \forall x \in \mathscr{D}(A)$;

v) $A 0$ is a linear subspace of $X$ and $A x=y+A 0$ for any $y \in A x, x \in \mathscr{D}(A)$. Thus, $A$ is single-valued if and only if $A 0=\{0\}$.

The inverse $A^{-1}$ of a m. 1. operator $A$ is the m. 1. operator having domain $\mathscr{D}\left(A^{-1}\right)=\mathscr{R}(A)$ and such that $A^{-1} y=\{x \in \mathscr{D}(A): y \in A x\}, y \in \mathscr{D}\left(A^{-1}\right)$. In particular, $\left(A^{-1}\right)^{-1}=A$ (cf. [12, Theorem 1.3]). The set $A^{-1} 0=\{x \in \mathscr{D}(A): 0 \in A x\}$ is called the kernel of $A$ and it is denoted by $\mathscr{N}(A)$. Thus, $\mathscr{N}\left(A^{-1}\right)=A 0$. If $\mathscr{N}(A)=\{0\}$, i.e. if $A^{-1}$ is single-valued, then $A$ is said to be injective. From v) it thus follows that $A x=A 0$ if and only if $x \in \mathscr{N}(A)$. Notice that, in general, from v) it follows that $A x=A y, x, y \in \mathscr{D}(A)$, if and only if $A x \cap A y \neq \varnothing$, but, if $A$ is injective, $A x=A y$ if and only if $x=y$.

If $A_{1}$ and $A_{2}$ are m. 1 . operators in $X$ and $z \in \mathbf{C}$, then the scalar multiplication $z A_{1}$, the sum $A_{1}+A_{2}$, and the product $A_{1} A_{2}$ are the m. 1. operators in $X$ defined as follows: 


$$
\left\{\begin{array}{l}
\mathscr{D}\left(z A_{1}\right)=\mathscr{D}\left(A_{1}\right), \quad\left(z A_{1}\right) x=z A_{1} x, x \in \mathscr{D}\left(z A_{1}\right), \\
\mathscr{D}\left(A_{1}+A_{2}\right)=\mathscr{D}\left(A_{1}\right) \cap \mathscr{D}\left(A_{2}\right), \quad\left(A_{1}+A_{2}\right) x=A_{1} x+A_{2} x, x \in \mathscr{D}\left(A_{1}+A_{2}\right), \\
\mathscr{D}\left(A_{1} A_{2}\right)=\left\{x \in \mathscr{D}\left(A_{2}\right): A_{1}\left(A_{2} x\right) \neq \varnothing\right\}, \quad\left(A_{1} A_{2}\right) x=A_{1}\left(A_{2} x\right), x \in \mathscr{D}\left(A_{1} A_{2}\right) .
\end{array}\right.
$$

It is verified that $(\zeta z) A_{1}=\zeta\left(z A_{1}\right), \zeta, z \in \mathbf{C}$, and (cf. [12, Section 1.2]) $\left(A_{1} A_{2}\right)^{-1}=$ $A_{2}^{-1} A_{1}^{-1}$. Moreover, denoting by $I$ the identity operator in $X$, from (2.1) we see that a m. 1. operator $A$ is injective (respectively, single-valued) if and only if $A^{-1} A=\left.I\right|_{\mathscr{D}(A)}$ (respectively, $\left.A A^{-1}=\left.I\right|_{\mathscr{R}(A)}\right)$.

Defining $A^{0}$ and $\mathscr{D}\left(A^{0}\right)$ to be $I$ and $X$, respectively, from the third relation in (2.1) we have that the integer powers $A^{n}, n \in \mathbf{N}=\{1,2, \ldots\}$, of a m. 1. operator $A$ are defined by induction in the following way:

$$
\left\{\begin{array}{l}
\mathscr{D}\left(A^{n}\right)=\left\{x \in \mathscr{D}\left(A^{n-1}\right): \mathscr{D}(A) \cap A^{n-1} x \neq \varnothing\right\}, \\
A^{n} x=\left(A A^{n-1}\right) x=\bigcup_{y \in \mathscr{D}(A) \cap A^{n-1} x} A y, \quad x \in \mathscr{D}\left(A^{n}\right) .
\end{array}\right.
$$

Hence, an induction argument leads to $\left(A^{n}\right)^{-1}=\left(A^{n-1}\right)^{-1} A^{-1}=\left(A^{-1}\right)^{n}, n \in \mathbf{N}$. Clearly, if $A$ is single-valued, (2.2) coincides with the usual definition for integer powers of single-valued operators, since $\mathscr{D}(A) \cap A^{n-1} x \neq \varnothing$ reduces to $A^{n-1} x \in \mathscr{D}(A)$.

If $A$ and $B$ are m. 1. operators in $X$ we write $A \subset B$ if $\mathscr{D}(A) \subseteq \mathscr{D}(B)$ and $A x \subseteq B x$ for every $x \in \mathscr{D}(A)$, where "ᄃ" must be understood in the set-theoretical meaning. If $A \subset B$ and $A x=B x$ for every $x \in \mathscr{D}(A)$, i.e. if $A=\left.B\right|_{\mathscr{D}(A)}$, then $B$ is said to be an extension of $A$. Clearly, $A \subset B \subset A$ is equivalent to $A=B$. Observe that $A \subset B$ if and only if $z A \subset z B, z \in \mathbf{C} \backslash\{0\}$. Indeed, if $A \subset B$, then $\mathscr{D}(z A)=\mathscr{D}(A) \subseteq \mathscr{D}(B)=\mathscr{D}(z B)$ and $(z A) x=z A x \subseteq z B x=(z B) x, x \in \mathscr{D}(z A)$, i.e. $z A \subset z B$. Vice versa, if $z A \subset z B, z \in \mathbf{C} \backslash\{0\}$, then from the first part it follows that $A=z^{-1}(z A) \subset z^{-1}(z B)=B$. If a linear single-valued operator $S$ has domain $\mathscr{D}(S)=\mathscr{D}(A)$ and $S \subset A$, then $S$ is called a section of $A$. With an arbitrary section $S$, it holds that $A x=S x+A 0, x \in \mathscr{D}(A)$, and $\mathscr{R}(A)=\mathscr{R}(S)+A 0$, but this latter sum may or may not be direct (cf. [6, p. 14]). A method for constructing sections is provided by [6, Proposition I.5.2].

If $\left(X_{j},\|\cdot\|_{X_{j}}\right), j=1,2$, are two complex Banach spaces, the linear space of all bounded single-valued linear operators $L$ from $X_{1}$ to $X_{2}$ is denoted by $\mathscr{L}\left(X_{1} ; X_{2}\right)$ and it is equipped with the uniform operator norm $\|L\|_{\mathscr{L}\left(X_{1} ; X_{2}\right)}=$ $\inf _{K \geq 0}\left\{\|L x\|_{X_{2}} \leq K\|x\|_{X_{1}}\right.$ for all $\left.x \in X_{1}\right\}, L \in \mathscr{L}\left(X_{1} ; X_{2}\right)$. For brevity, $\mathscr{L}\left(X_{1} ; X_{1}\right)$ $=\mathscr{L}\left(X_{1}\right)$. Then, if $A$ is a m. 1 . operator in $X$, the resolvent set $\rho(A)$ of $A$ is defined to be the set of all $z \in \mathbf{C}$ such that $\mathscr{D}\left((z I-A)^{-1}\right)=X$ and $(z I-A)^{-1} \in$ $\mathscr{L}(X)$. The basic properties of the resolvent set of single-valued operators hold 
the same for m. 1. operators. First, if $\rho(A) \neq \varnothing$, then the m. 1. operator $A$ is closed, that is its graph $\mathscr{G}(A)$ is closed in $X \times X$ (cf. [6, p. 43]). Further (cf. [12, Theorem 1.6]), $\rho(A)$ is an open set of $\mathbf{C}$ and the operator function $z \in \rho(A) \rightarrow$ $(z I-A)^{-1} \in \mathscr{L}(X)$ is holomorphic. Finally (cf. [12, Theorem 1.8]), the resolvent equation $(\lambda I-A)^{-1}-(\mu I-A)^{-1}=(\mu-\lambda)(\lambda I-A)^{-1}(\mu I-A)^{-1}, \lambda, \mu \in \rho(A)$, is satisfied, too. Unlike the single-valued case, instead, it holds that (cf. [12, Theorem 1.7]):

$$
(z I-A)^{-1} A \subset z(z I-A)^{-1}-I \subset A(z I-A)^{-1}, \quad z \in \rho(A) .
$$

Then, in general, $z(z I-A)^{-1}-I, z \in \rho(A)$, is only a bounded section of the $\mathrm{m}$. 1 . operator $A(z I-A)^{-1}$. Throughout the paper this bounded section is denoted by $A^{\circ}(z I-A)^{-1}$, but here $A^{\circ}$ is only a symbol and does not necessarily denote a section of $A$ itself. Of course, if $0 \in \rho(A)$, then $A^{\circ}(0 I-A)=-I$ by definition and, if $A$ is single-valued, $A^{\circ}(z I-A)^{-1}$ reduces to $A(z I-A)^{-1}$. Notice that the first inclusion in (2.3) implies that $(z I-A)^{-1} A, z \in \rho(A)$, is single-valued on $\mathscr{D}(A)$ and $(z I-A)^{-1} A x=(z I-A)^{-1} y$ for every $y \in A x, x \in \mathscr{D}(A)$. Another difference with the single-valued case is that for every $z \in \rho(A)$ it holds $\mathscr{N}\left((z I-A)^{-1}\right)=$ $(z I-A) 0=A 0$. Therefore, in the really multivalued case, $\{0\} \subsetneq \mathscr{N}\left((z I-A)^{-1}\right)$ for every $z \in \rho(A)$.

We recall that, if $0 \in \rho(A)$, then from [11, Lemma 5.1 with $\left.T=A^{-1}\right]$ we have

$$
(z I-A)^{-1}=A^{-1}\left(z A^{-1}-I\right)^{-1}=\left(z A^{-1}-I\right)^{-1} A^{-1}, \quad \forall z \in \mathbf{C},
$$

in the sense of m. 1 . operator. As a consequence, when $0 \in \rho(A)$, we have also the following useful characterization of $A^{\circ}(z I-A)^{-1}$ (cf. [11, p. 375]):

$$
A^{\circ}(z I-A)^{-1}=\left(z A^{-1}-I\right)^{-1}, \quad \forall z \in \rho(A) .
$$

Thus, if $0 \in \rho(A)$, combining (2.4) and (2.5) we get

$$
A^{-1} A^{\circ}(z I-A)^{-1}=A^{-1}\left(z A^{-1}-I\right)^{-1}=(z I-A)^{-1}, \quad \forall z \in \rho(A) .
$$

Lemma 2.1. Let $0 \in \rho(A)$. Then $\mathscr{N}\left(A^{\circ}(z I-A)^{-1}\right)=\{0\}$ for every $z \in \rho(A)$. In addition, $x \notin A 0$ if and only if $A^{\circ}(z I-A)^{-1} x \notin A 0, z \in \rho(A)$.

Proof. Let $z \in \rho(A)$ and assume $x \in \mathscr{N}\left(A^{\circ}(z I-A)^{-1}\right)$. Then $0=$ $A^{\circ}(z I-A)^{-1} x=z(z I-A)^{-1} x-x$, so that $z(z I-A)^{-1} x=x$. This implies $x \in \mathscr{D}(A)$, for $(z I-A)^{-1} x \in \mathscr{D}(A)$ and $\mathscr{D}(A)$ is a linear subspace of $X$. Therefore, $z x \in(z I-A) x=\{z x-y: y \in A x\}$ which leads to $0 \in A x$, i.e. $x \in \mathscr{N}(A)$. Since $A$ is injective, we thus find $x=0$, completing the proof of $\mathscr{N}\left(A^{\circ}(z I-A)^{-1}\right)=\{0\}$. 
Let us now prove that $x \notin A 0$ if and only if $A^{\circ}(z I-A)^{-1} x \notin A 0, z \in \rho(A)$. Assume first $x \notin A 0$. If for some $z \in \rho(A)$ it holds that $A^{\circ}(z I-A)^{-1} x \in A 0$, then from (2.6) it follows that $0=A^{-1} A^{\circ}(z I-A)^{-1} x=(z I-A)^{-1} x$, i.e. $x \in$ $\mathscr{N}\left((z I-A)^{-1}\right)=A 0$, a contradiction. Vice versa, if $x \in A 0$, then $A^{\circ}(z I-A)^{-1} x$ $=z(z I-A)^{-1} x-x=-x \in A 0$.

LeMmA 2.2. For every $\lambda, \mu \in \rho(A)$ it holds that

$$
\begin{aligned}
& (\mu-\lambda)(\lambda I-A)^{-1} A^{\circ}(\mu I-A)^{-1}=A^{\circ}(\lambda I-A)^{-1}-A^{\circ}(\mu I-A)^{-1}, \\
& (\mu-\lambda) A^{\circ}(\lambda I-A)^{-1}(\mu I-A)^{-1}=A^{\circ}(\lambda I-A)^{-1}-A^{\circ}(\mu I-A)^{-1},
\end{aligned}
$$

Proof. First, since $A^{\circ}(\mu I-A)^{-1}=\mu(\mu I-A)^{-1}-I$, we have

$$
\begin{aligned}
& (\mu-\lambda)(\lambda I-A)^{-1} A^{\circ}(\mu I-A)^{-1} \\
& \quad=\mu(\mu-\lambda)(\lambda I-A)^{-1}(\mu I-A)^{-1}-(\mu-\lambda)(\lambda I-A)^{-1} .
\end{aligned}
$$

Then, applying the resolvent equation to the right-hand side of (2.9), we get

$$
\begin{aligned}
(\mu-\lambda) & (\lambda I-A)^{-1} A^{\circ}(\mu I-A)^{-1} \\
= & \mu(\lambda I-A)^{-1}-\mu(\mu I-A)^{-1}-(\mu-\lambda)(\lambda I-A)^{-1} \\
= & {\left[\lambda(\lambda I-A)^{-1}-I\right]-\left[\mu(\mu I-A)^{-1}-I\right] . }
\end{aligned}
$$

This completes the proof of (2.7). To prove (2.8) it suffices to write $A^{\circ}(\lambda I-A)^{-1}$ $=\lambda(\lambda I-A)^{-1}-I$ and to proceed as in the proof of $(2.7)$.

Let $A$ be a m. 1. operator in $X$ satisfying the following resolvent condition:

(H1) $\rho(A)$ contains a region $\Sigma_{\alpha}=\left\{z \in \mathbf{C}: \Re \mathrm{e} z \geq-c(|\Im \mathrm{m} z|+1)^{\alpha}, \Im \mathrm{m} z \in \mathbf{R}\right\}$, $\alpha \in(0,1], c>0$, and for some exponent $\beta \in(0, \alpha]$ and constant $C>0$ it holds:

$$
\left\|(\lambda I-A)^{-1}\right\|_{\mathscr{L}(X)} \leq C(|\lambda|+1)^{-\beta}, \quad \forall \lambda \in \Sigma_{\alpha} .
$$

Assumption (H1), said of abstract weak parabolicity, implies that $A$ generates an infinitely strongly differentiable semigroup of bounded linear operators on $X$. Precisely, introduce the family $\left\{\mathrm{e}^{t A}\right\}_{t \geq 0} \subset \mathscr{L}(X)$ defined by $\mathrm{e}^{0 A}=I$ and

$$
\mathrm{e}^{t A}=\frac{1}{2 \pi i} \int_{\Gamma} \mathrm{e}^{t \lambda}(\lambda I-A)^{-1} \mathrm{~d} \lambda, \quad t>0
$$


$\Gamma \subset \Sigma_{\alpha} \backslash\{z \in \mathbf{C}: \Re \mathrm{e} z \geq 0\}$ being the contour parametrized by $\lambda=-c(|\eta|+1)^{\alpha}+$ $i \eta, \eta \in(-\infty, \infty)$. We shall denote by $\Gamma_{ \pm}$the infinite branches $\{\lambda \in \Gamma: \pm \Im \mathrm{m} z>0\}$ of $\Gamma$ such that $\Gamma=\Gamma_{+} \cup \Gamma_{-} \cup\{-c\}$. Then, according to [11, Section 3] for the multivalued case and to [32, Theorem 5.1] for the single-valued one, $\left\{\mathrm{e}^{t A}\right\}_{t \geq 0}$ is a semigroup on $X$, infinitely many times strongly differentiable for $t>0$ with

$$
D_{t}^{k} \mathrm{e}^{t A}=\frac{1}{2 \pi i} \int_{\Gamma} \lambda^{k} \mathrm{e}^{t \lambda}(\lambda I-A)^{-1} \mathrm{~d} \lambda, \quad t>0, k \in \mathbf{N} .\left(D_{t}^{k}=\frac{\mathrm{d}^{k}}{\mathrm{~d} t^{k}}\right)
$$

In general, no analiticity should be expected for $\mathrm{e}^{t A}$. For, if $\alpha<1$ in (H1), then $\Sigma_{\alpha}$ does not contain any sector $\Lambda_{\omega+\pi / 2}=\{z \in \mathbf{C} \backslash\{0\}:|\arg z|<\omega+\pi / 2\}, \omega \in$ $(0, \pi / 2)$, and [32, Theorem 5.3], which extends $\mathrm{e}^{t A}$ to an analytic semigroup in the sector $\Lambda_{\omega}$ containing the positive real axis, is not applicable.

We stress that (2.10) and $\mathscr{N}\left((z I-A)^{-1}\right)=A 0, z \in \rho(A)$, imply $A 0 \subset \mathscr{N}\left(\mathrm{e}^{t A}\right)$ for every $t>0$, whereas $\mathscr{N}\left(\mathrm{e}^{0 A}\right)=\mathscr{N}(I)=\{0\}$. Hence, if $A$ is really a $\mathrm{m}$. 1 . operator, then $\mathrm{e}^{t A}$ has definitely nonzero kernel for $t>0$ and the following inclusion holds:

$$
A 0 \subseteq \bigcap_{t>0} \mathscr{N}\left(\mathrm{e}^{t A}\right)
$$

From the semigroup property it also follows $\mathscr{N}\left(\mathrm{e}^{t_{0} A}\right) \subseteq \mathscr{N}\left(\mathrm{e}^{t_{1} A}\right)$ for $t_{1} \geq t_{0} \geq 0$.

We shall come back in Section 3 to the semigroup $\mathrm{e}^{t A}$, after having introduced the operators $\left[(-A)^{\theta}\right]^{\circ} \mathrm{e}^{t A} \in \mathscr{L}(X), \theta \geq 0, t>0$. Here, instead, we want only to recall that under assumption $(\mathrm{H} 1)$ we can specify a topology on $\mathscr{D}(A)$ equipping it with the norm

$$
\|x\|_{\mathscr{D}(A)}=\inf _{y \in A x}\|y\|_{X}, \quad x \in \mathscr{D}(A) .
$$

Since $A^{-1}$ belongs to $\mathscr{L}(X)$ this norm is equivalent to the graph norm and makes $\mathscr{D}(A)$ a complex Banach space (cf. [12, Proposition 1.11]). From now on, $X_{1}$ and $X_{2}$ being given normed complex linear spaces, we shall say that $X_{1}$ is continuously embedded in $X_{2}$, and we shall write $X_{1} \hookrightarrow X_{2}$, if $X_{1} \subseteq X_{2}$ and there exists a positive constant $C_{1}$ such that $\|x\|_{X_{2}} \leq C_{1}\|x\|_{X_{1}}$ for every $x \in X_{1}$. Then, $\mathscr{D}(A)$ endowed with the norm (2.13) satisfies $\mathscr{D}(A) \hookrightarrow X$. In fact, if $x \in \mathscr{D}(A)$, then $x=A^{-1} y$ for every $y \in A x$ so that $\|x\|_{X} \leq\left\|A^{-1}\right\|_{\mathscr{L}(X)}\|y\|_{X} \leq C\|y\|_{X}$ for every $y \in A x$. Taking the infimum with respect to $y \in A x$ in the latter inequality, we thus find $\|x\|_{X} \leq C\|x\|_{\mathscr{D}(A)}$ for every $x \in \mathscr{D}(A)$.

\section{The Fractional Powers $(-A)^{ \pm \theta}$ for $\Re$ e $\theta>1-\beta$}

Let $A$ be a m. 1. operator in $X$ satisfying assumption (H1) for some $\beta \in(0, \alpha]$, $\alpha \in(0,1]$. Then, according to [12, Section 1.4] which generalizes [32, Section 6] 
to m. 1. operators, we define the fractional powers $(-A)^{-\theta}$ of $-A$ by the integrals

$$
(-A)^{-\theta}=\frac{1}{2 \pi i} \int_{\Gamma}(-\lambda)^{-\theta}(\lambda I-A)^{-1} \mathrm{~d} \lambda, \quad \theta \in \mathbf{C}, \Re \mathrm{e} \theta>1-\beta .
$$

Here the contour $\Gamma$ avoiding the origin and the positive real axis is the same occurring in formula (2.10), and for the function $(-\lambda)^{-\theta}=\mathrm{e}^{-\theta \log (-\lambda)}$ we choose the principal branch holomorphic in the region $\mathbf{C} \backslash\{z \in \mathbf{C}: \Re \mathrm{e} z \geq 0\}$, where for principal branch we mean that the principal determination $\ln |z|+i \arg (z)$, $\arg (z) \in(-\pi, \pi)$, of $\log (z)$ is considered. We like to mention that in the case $A \in \mathscr{L}(X)$ definition (3.1) coincide with that given in [19, Section 17.7], where the theory of fractional powers in the framework of operational calculus originated. Now, for every $\lambda \in \Gamma$ and every $\zeta \in \mathbf{C}$ it holds that

$$
\left|(-\lambda)^{\zeta}\right|=\left|\mathrm{e}^{\zeta \log (-\lambda)}\right|=|\lambda|^{\Re \mathrm{e} \zeta} \mathrm{e}^{-\Im \mathrm{m} \zeta \arg (-\lambda)} \leq|\lambda|^{\Re \mathrm{e} \zeta} \mathrm{e}^{(\pi / 2)|\Im \mathrm{m} \zeta|} .
$$

Then, since $|\lambda| \geq c$ for $\lambda \in \Gamma$, for $\Re$ e $\theta>1-\beta$ from (3.1) and (3.2) we have:

$$
\begin{aligned}
\left\|(-A)^{-\theta} x\right\|_{X} & \leq C \mathrm{e}^{(\pi / 2)|\Im \mathrm{m} \theta|}\left[\int_{\Gamma}|\lambda|^{-\Re \mathrm{e} \theta}(|\lambda|+1)^{-\beta}|\mathrm{d} \lambda|\right]\|x\|_{X} \\
& \leq 2 C \mathrm{e}^{(\pi / 2)|\Im \mathrm{m} \theta|}\left[\int_{c}^{\infty} \eta^{-\Re \mathrm{e} \theta-\beta} \mathrm{d} \eta\right]\|x\|_{X} \\
& =2 C \mathrm{e}^{(\pi / 2)|\Im \mathrm{m} \theta|}(\Re \mathrm{e} \theta+\beta-1)^{-1} c^{-\Re \mathrm{e} \theta-\beta+1}\|x\|_{X} \quad \forall x \in X .
\end{aligned}
$$

It thus follows that $(-A)^{-\theta} \in \mathscr{L}(X)$ for every $\theta \in \mathbf{C}$ such that $\Re$ e $\theta>1-\beta$. Notice that, $\beta$ being positive, the value $\Re$ e $\theta=1$ is admitted. In particular, (3.1) with $\theta=1$ leads to $(-A)^{-1}=(2 \pi i)^{-1} \int_{\Gamma}(-\lambda)^{-1}(\lambda I-A)^{-1} \mathrm{~d} \lambda$ which is precisely the Cauchy's formula for the holomorphic function $\mu \in \rho(A) \rightarrow(\mu I-A)^{-1} \in$ $\mathscr{L}(X)$. We recall briefly the main properties of $(-A)^{-\theta}$, $\Re$ e $\theta>1-\beta$. First (cf. $[8$, p. 252]), using the resolvent equation and the residue theorem we can easily verify the semigroup property $(-A)^{-\theta}(-A)^{-\theta^{\prime}}=(-A)^{-\left(\theta+\theta^{\prime}\right)}, \Re \mathrm{e} \theta, \Re \mathrm{e} \theta^{\prime}>1-\beta$. Then, the same proof as in [32, Proposition 6.1(ii)], shows that $(-A)^{-n}=$ $\left((-A)^{-1}\right)^{n}, n \in \mathbf{N}$. Finally, applying the Cauchy theorem to deform the infinite branches $\Gamma_{+}$and $\Gamma_{-}$of $\Gamma$ into the upper and lower sides of the positive real axis, respectively, in [32, Theorem 6.2] it is shown that

$$
(-A)^{-\theta}=\frac{\sin (\theta \pi)}{\pi} \int_{0}^{\infty} s^{-\theta}(s I-A)^{-1} \mathrm{~d} s, \quad \Re \mathrm{e} \theta \in(1-\beta, 1) .
$$


REMARK 3.1. Let assume here $\beta=1$. Then, reasoning as in [26, p. 281], it is possible to assign a meaning to (3.3) for all $\theta$ in the half-plane $\{z \in \mathbf{C}: \Re \mathrm{e} z>0\}$. Indeed, integrating by parts the right-hand side of (3.3) and using the formula $\frac{\mathrm{d}^{k}}{\mathrm{~d} \lambda^{k}}(\lambda I-A)^{-1}=(-1)^{k} k !\left[(\lambda I-A)^{-1}\right]^{k+1}, \quad k \in \mathbf{N}, \lambda \in \rho(A)$, for $\Re \mathrm{e} \theta \in(0,1)$ we obtain

$$
\begin{aligned}
(-A)^{-\theta} & =\frac{\sin (\theta \pi)}{\pi(1-\theta)}\left\{\left.s^{1-\theta}(s I-A)^{-1}\right|_{0} ^{\infty}+\int_{0}^{\infty} s^{1-\theta}\left[(s I-A)^{-1}\right]^{2} \mathrm{~d} s\right\} \\
& =\frac{\sin (\theta \pi)}{\pi(1-\theta)} \int_{0}^{\infty} s^{1-\theta}\left[(s I-A)^{-1}\right]^{2} \mathrm{~d} s .
\end{aligned}
$$

The latter integral actually converges in the $\mathscr{L}(X)$-norm for $\Re$ e $\theta \in(0,2)$ and the coefficients in this integral are entire functions. Hence (3.4) gives an analytic continuation of (3.3) to the infinite vertical open strip $\{z \in \mathbf{C}: \Re \mathrm{e} z \in(0,2)\}$. Repeating the previous argument, by $m$ successive integration by parts, $m \in \mathbf{N}$, we obtain

$$
(-A)^{-\theta}=\frac{m ! \sin (\theta \pi)}{\pi \Pi_{k=1}^{m}(k-\theta)} \int_{0}^{\infty} s^{m-\theta}\left[(s I-A)^{-1}\right]^{m+1} \mathrm{~d} s, \quad \Re \mathrm{e} \theta \in(0, m+1) .
$$

Each formula in (3.5) defines an analytic continuation of (3.4) to the strip $\{z \in \mathbf{C}: \Re \mathrm{e} z \in(0, m+1)\}, m \in \mathbf{N}$, and we may consider (3.3) to be defined for all $\theta \in\{z \in \mathbf{C}: \Re \mathrm{e} z>0\}$.

REMARK 3.2. If $\beta \in(0,1)$, then it is not possible to repeat the argument in Remark 3.1. Indeed, if $\beta \in(0,1)$, then the integral on the right-hand side of (3.4) requires $\Re \mathrm{e} \theta \in(2(1-\beta), 2)$ to converge in the norm of $\mathscr{L}(X)$. This means that (3.3) and (3.4) may both take sense, but for possibly non overlapping intervals. Thus, we can not consider (3.4) as an analytic continuation of (3.3). Similarly, the integral in (3.5) needs $\Re$ e $\theta \in((m+1)(1-\beta), m+1)$ for converging in the norm of $\mathscr{L}(X)$. For this reason, in the case $\beta \in(0,1)$, the representation (3.1) of $(-A)^{-\theta}$ is more convenient than (3.3).

Observe that (3.1) is meaningless in the closed strip $\Delta_{1-\beta}=\{z \in \mathbf{C}: \Re \mathrm{e} z \in$ $[0,1-\beta]\}$, for the integral on the right-hand side becomes singular at infinity for $\theta \in \Delta_{1-\beta}$. In particular, when $\beta=1, \Delta_{0}$ coincides with the imaginary axis and, according to the results in [23, Section 5], [25, Section 1] and [35, Section 1.15] for the single-valued case, (3.1) provides the fractional powers $(-A)^{-\theta} \in \mathscr{L}(X)$ of $-A$ only for $\Re$ e $\theta>0$. 
The case $\beta=1$ is explicit and we assume it for a moment. Contrarily to the single-valued case and as we shall make clearer in Remark 3.8 below, here the multivalued character of $A$ prevents us to proceed as in [23, p. 305] for defining the pure imaginary powers of $-A$. This fact highlights the peculiar feature of m. 1. operators and, at the same time, suggests that all the abstract and concrete results which contain assumptions on the behavior of the pure imaginary powers of single-valued linear operators may fail in the multivalued case. We refer in particular to [2, Lemma 3.2, Theorem 3.3, Proposition 4.2 and Section 7], [7, Theorems 2.1, 3.1-3.3 and Corollary 2.9], [16, Section 6], [30, 31, Section 3], [24, Proposition 2.9] and [35, Theorem 1.15.3]).

Later on, in Section 9, we shall provide a possible approach for defining $(-A)^{-\theta}$ also for $\theta \in \Delta_{1-\beta}$. If $A$ is a single-valued densely defined linear closed operator and $\beta=1$, then our attempt will be in accordance with [24, Proposition 2.9], but in our general case it will furnish the fractional powers $(-A)^{-\theta}, \theta \in \Delta_{1-\beta}$, only as a class of $\mathrm{m}$. 1 . operators.

Now, for $\Re$ e $\theta>1-\beta$, we define $(-A)^{\theta}$ as the inverse $\left((-A)^{-\theta}\right)^{-1}$ of $(-A)^{-\theta}$. Thus

$$
\left\{\begin{array}{l}
\mathscr{D}\left((-A)^{\theta}\right)=\mathscr{R}\left((-A)^{-\theta}\right)=\left\{x \in X: x=(-A)^{-\theta} y, y \in X\right\}, \\
(-A)^{\theta} x=\left\{y \in X: x=(-A)^{-\theta} y\right\}, \quad x \in \mathscr{D}\left((-A)^{\theta}\right) .
\end{array}\right.
$$

Clearly, from $\left((-A)^{\theta}\right)^{-1}=\left(\left((-A)^{-\theta}\right)^{-1}\right)^{-1}=(-A)^{-\theta} \in \mathscr{L}(X)$ we have $0 \in$ $\rho\left((-A)^{\theta}\right)$, and $(-A)^{\theta}$ turns out to be a closed m. 1. operator. Also, in both the single- and multivalued cases, if $\beta \in(1 / 2, \alpha], \alpha \in(1 / 2,1]$, it holds that (cf. [32, Theorem 6.5] and [8, p. 252]):

$$
\mathscr{D}(A) \subseteq \mathscr{D}\left((-A)^{\theta}\right), \quad \Re \mathrm{e} \theta \in(1-\beta, \beta) .
$$

Indeed, if $x \in \mathscr{D}(A)$ and $\Re$ e $\theta \in(1-\beta, \beta)$, then $x=A^{-1} y$ for every $y \in A x$ and we can define both $(-A)^{-\theta}$ and $(-A)^{-(1-\theta)}$. The assertion now follows from $x=$ $A^{-1} y=(-A)^{-\theta}\left((-A)^{-(1-\theta)} y\right), y \in A x$, which proves $x \in \mathscr{R}\left((-A)^{-\theta}\right)=\mathscr{D}\left((-A)^{\theta}\right)$. Similarly,

$$
\mathscr{D}\left((-A)^{\theta_{1}}\right) \subseteq \mathscr{D}\left((-A)^{\theta_{2}}\right), \quad 1-\beta<\Re \mathrm{e} \theta_{2}<\Re \mathrm{e} \theta_{1}+\beta-1 .
$$

In fact, in this case $(-A)^{-\theta_{2}}$ and $(-A)^{-\left(\theta_{1}-\theta_{2}\right)}$ are both well-defined and the semigroup property leads to $(-A)^{-\theta_{1}}=(-A)^{-\theta_{2}}(-A)^{-\left(\theta_{1}-\theta_{2}\right)}$, that is $\mathscr{D}\left((-A)^{\theta_{1}}\right)=$ $\mathscr{R}\left((-A)^{-\theta_{1}}\right) \subseteq \mathscr{R}\left((-A)^{-\theta_{2}}\right)=\mathscr{D}\left((-A)^{\theta_{2}}\right)$. Notice that $(3.8)$ with $\left(\theta_{1}, \theta_{2}\right)=(1, \theta)$ is precisely (3.7). 
For $\Re \mathrm{e} \theta>1-\beta$, from (2.1) and (3.6) it follows that:

$$
\left\{\begin{array}{l}
\mathscr{D}\left((-A)^{-\theta}(-A)^{\theta}\right)=\left\{x \in \mathscr{D}\left((-A)^{\theta}\right): X \cap(-A)^{\theta} x \neq \varnothing\right\}=\mathscr{D}\left((-A)^{\theta}\right), \\
\left((-A)^{-\theta}(-A)^{\theta}\right) x=\bigcup_{y \in(-A)^{\theta} x}(-A)^{-\theta} y=x, \quad \forall x \in \mathscr{D}\left((-A)^{\theta}\right),
\end{array}\right.
$$

whereas

$$
\left\{\begin{array}{l}
\mathscr{D}\left((-A)^{\theta}(-A)^{-\theta}\right)=\left\{y \in X:(-A)^{-\theta} y \in \mathscr{D}\left((-A)^{\theta}\right)\right\}=X, \\
\left((-A)^{\theta}(-A)^{-\theta}\right) y=\bigcup_{x=(-A)^{-\theta} y}(-A)^{\theta} x \supseteq\{y\}, \quad \forall y \in X .
\end{array}\right.
$$

Therefore, for $\Re \mathrm{e} \theta>1-\beta$, we have:

$$
\begin{cases}I=(-A)^{-\theta}(-A)^{\theta}, & \text { on } \mathscr{D}\left((-A)^{\theta}\right), \\ I \subset(-A)^{\theta}(-A)^{-\theta}, & \text { on } X .\end{cases}
$$

We stress that, if $A$ is single-valued, then [32, Proposition 6.(iii)] applies and $(-A)^{-\theta}$ is injective for $\Re \mathrm{e} \theta>1-\beta$. Thus $(-A)^{\theta}$ is single-valued and the second of (3.9) becomes an equality. This is a remarkable fact, since, as we shall see in Remark 3.7 for the case $\beta=1$, the usual definition of $(-A)^{\theta}$, $\Re$ e $\theta>0$, for singlevalued linear operators leads to $(-A)^{\theta}(-A)^{-\theta}=I$ only on $\overline{\mathscr{D}(A)}$, closure of $\mathscr{D}(A)$ in $X$. On the contrary, since (3.1) implies $A 0 \subseteq \mathscr{N}\left((-A)^{-\theta}\right)$, the injectivity of $(-A)^{-\theta}$, or, equivalently, the single-valuedness of $(-A)^{\theta}$, is far from being true in the really multivalued case. Further, observe that $\mathscr{D}\left((-A)^{\theta}\right) \cap A 0$ may properly contain $\{0\}$ and that, if $x \in \mathscr{D}\left((-A)^{\theta}\right) \cap A 0$, then $x=(-A)^{-\theta} y$ with $y \in \mathscr{N}\left((-A)^{-(1+\theta)}\right)$. For, $0=-A^{-1} x=(-A)^{-1}(-A)^{-\theta} y=(-A)^{-(1+\theta)} y$.

The semigroup property does not hold in general, since the operators $(-A)^{\theta_{1}}(-A)^{\theta_{2}}$ and $(-A)^{\theta_{1}+\theta_{2}}$ may be very different. In fact, for $\Re \mathrm{e} \theta_{j}>1-\beta$, $j=1,2$, we have:

$$
\left\{\begin{aligned}
\mathscr{D}\left((-A)^{\theta_{1}}(-A)^{\theta_{2}}\right) & =\left\{x \in \mathscr{D}\left((-A)^{\theta_{2}}\right): \mathscr{D}\left((-A)^{\theta_{1}}\right) \cap(-A)^{\theta_{2}} x \neq \varnothing\right\} \\
& =\left\{x \in \mathscr{R}\left((-A)^{-\theta_{2}}\right): \mathscr{R}\left((-A)^{-\theta_{1}}\right) \cap(-A)^{\theta_{2}} x \neq \varnothing\right\}, \\
\left((-A)^{\theta_{1}}(-A)^{\theta_{2}}\right) x & =\bigcup_{y \in \mathscr{R}\left((-A)^{-\theta_{1}}\right) \cap(-A)^{\theta_{2}} x}(-A)^{\theta_{1}} y, \quad \forall x \in D\left((-A)^{\theta_{1}}(-A)^{\theta_{2}}\right),
\end{aligned}\right.
$$

but

$$
\left\{\begin{array}{l}
\mathscr{D}\left((-A)^{\theta_{1}+\theta_{2}}\right)=\mathscr{R}\left((-A)^{-\left(\theta_{1}+\theta_{2}\right)}\right), \\
(-A)^{\theta_{1}+\theta_{2}} x=\left\{y \in X: x=(-A)^{-\left(\theta_{1}+\theta_{2}\right)} y\right\}, \quad \forall x \in D\left((-A)^{\theta_{1}+\theta_{2}}\right) .
\end{array}\right.
$$

Notice that from (3.8) we have $\mathscr{D}\left((-A)^{\theta_{1}+\theta_{2}}\right) \subseteq \bigcap_{j=1}^{2} \mathscr{D}\left((-A)^{\theta_{j}}\right)$, $\Re$ e $\theta_{j}>1-\beta$, $j=1,2$. In fact $\Re \mathrm{e}\left(\theta_{1}+\theta_{2}\right)+\beta-1=\Re \mathrm{e} \theta_{1}+\Re \mathrm{e} \theta_{2}+\beta-1>\Re \mathrm{e} \theta_{j}>1-\beta, j=$ 1,2 . We are then able to exhibit a special situation in which the semigroup property holds. This is when $x \in \mathscr{D}\left((-A)^{\theta_{1}+\theta_{2}}\right), \Re \mathrm{e} \theta_{j}>1-\beta, j=1,2$, is such that $(-A)^{\theta_{2}} x$, which is well defined due to the previous argument, is contained in 
$\mathscr{D}\left((-A)^{\theta_{1}}\right)$. In this case, using (3.6) and the semigroup property for the negative fractional powers we obtain

$$
\begin{aligned}
\left((-A)^{\theta_{1}}(-A)^{\theta_{2}}\right) x & =\bigcup_{y \in \mathscr{D}\left((-A)^{\theta_{1}}\right) \cap(-A)^{\theta_{2} x}}(-A)^{\theta_{1}} y=\bigcup_{y \in(-A)^{\theta_{2}}}(-A)^{\theta_{1}} y \\
& =\bigcup_{y \in(-A)^{\theta_{2}}}\left\{w \in X: y=(-A)^{-\theta_{1}} w\right\} \\
& =\left\{w \in X: y=(-A)^{-\theta_{1}} w, y \in(-A)^{\theta_{2}} x\right\} \\
& =\left\{w \in X: y=(-A)^{-\theta_{1}} w, x=(-A)^{-\theta_{2}} y\right\} \\
& =\left\{w \in X: x=(-A)^{-\theta_{2}}(-A)^{-\theta_{1}} w\right\} \\
& =\left\{w \in X: x=(-A)^{-\left(\theta_{2}+\theta_{1}\right)} w\right\}=(-A)^{\theta_{2}+\theta_{1}} x .
\end{aligned}
$$

If $A$ is a single-valued densely defined linear operator satisfying (H1) with $\beta=1$, then the semigroup property $(-A)^{\theta+\theta^{\prime}} x=(-A)^{\theta}(-A)^{\theta^{\prime}} x$ holds assuming only that both side are well defined, and this is precisely when $x \in \mathscr{D}\left((-A)^{\theta+\theta^{\prime}}\right)$ or when $x \in \mathscr{D}\left((-A)^{\theta^{\prime}}\right)$ is such that $(-A)^{\theta^{\prime}} x \in \mathscr{D}\left((-A)^{\theta}\right)$. This is shown in [23, Proposition 5.2] and [35, Theorem 1.15.2], but using arguments and formulae that can not be employed in the really multivalued case (see the following Remark 3.5).

Similarly to what done for $\mathscr{D}(A)$ in (2.13), a topology can be specified on the domain $\mathscr{D}\left((-A)^{\theta}\right)$ of $(-A)^{\theta}, \Re \mathrm{e} \theta>1-\beta$, by equipping it with the norm

$$
\|x\|_{\mathscr{D}\left((-A)^{\theta}\right)}=\inf _{y \in(-A)^{\theta} x}\|y\|_{X}, \quad x \in \mathscr{D}\left((-A)^{\theta}\right) .
$$

Since $\left((-A)^{\theta}\right)^{-1}=(-A)^{-\theta} \in \mathscr{L}(X)$, then, replacing $A$ with $(-A)^{\theta}$ in [12, Proposition 1.11], we see that (3.10) is equivalent to the graph norm and makes $\mathscr{D}\left((-A)^{\theta}\right)$ a complex Banach space. Also, $\mathscr{D}\left((-A)^{\theta}\right) \hookrightarrow X$. Indeed, $\|x\|_{X}=$ $\left\|(-A)^{-\theta} y\right\|_{X} \leq\left\|(-A)^{-\theta}\right\|\left\|_{\mathscr{L}(X)}\right\| y \|_{X}$ for every $y \in(-A)^{\theta} x, x \in \mathscr{D}\left((-A)^{\theta}\right)$, so that, passing to the infimum with respect to $y \in(-A)^{\theta} x$, it follows

$$
\|x\|_{X} \leq\left\|(-A)^{-\theta}\right\|_{\mathscr{L}(X)}\|x\|_{\mathscr{D}\left((-A)^{\theta}\right)}, \quad x \in \mathscr{D}\left((-A)^{\theta}\right) .
$$

When $\mathscr{D}\left((-A)^{\theta}\right)$ is endowed with the norm (3.10) inclusions (3.8) become embeddings.

Proposition 3.3. Let $A$ be a m. l. operator in $X$ satisfying (H1). Then

$$
\mathscr{D}\left((-A)^{\theta_{1}}\right) \hookrightarrow \mathscr{D}\left((-A)^{\theta_{2}}\right), \quad 1-\beta<\Re \mathrm{e} \theta_{2}<\Re \mathrm{e} \theta_{1}+\beta-1 .
$$

In particular, if $\beta>1 / 2$, then $\mathscr{D}(A) \hookrightarrow \mathscr{D}\left((-A)^{\theta}\right)$, $\Re \mathrm{e} \theta \in(1-\beta, \beta)$. 
Proof. Let $x \in \mathscr{D}\left((-A)^{\theta_{1}}\right)$. Then, $x=(-A)^{-\theta_{1}} y=(-A)^{-\theta_{2}}\left((-A)^{-\left(\theta_{1}-\theta_{2}\right)} y\right)$, for every $y \in(-A)^{\theta_{1}} x$ and for every $\theta_{1}, \theta_{2} \in \mathbf{C}$ such that $1-\beta<\Re \mathrm{e} \theta_{2}<$ $\Re$ e $\theta_{1}+\beta-1$. Hence (cf. the second in (3.9)), $(-A)^{-\left(\theta_{1}-\theta_{2}\right)} y \in(-A)^{\theta_{2}} x$, for every $y \in(-A)^{\theta_{1}} x$, so that

$$
\|x\|_{\mathscr{D}\left((-A)^{\left.\theta_{2}\right)}\right.}=\inf _{w \in(-A)^{\theta_{2}}}\|w\|_{X} \leq\left\|(-A)^{-\left(\theta_{1}-\theta_{2}\right)}\right\|_{\mathscr{L}(X)}\|y\|_{X}, \quad \forall y \in(-A)^{\theta_{1}} x .
$$

Passing to the infimum with respect to $y \in(-A)^{\theta_{1}} x$ in the latter inequality, we get $\|x\|_{\mathscr{D}\left((-A)^{\left.\theta_{2}\right)}\right.} \leq\left\|(-A)^{-\left(\theta_{1}-\theta_{2}\right)}\right\|_{\mathscr{L}(X)}\|x\|_{\mathscr{D}\left((-A)^{\theta_{1}}\right)}, x \in \mathscr{D}\left((-A)^{\theta_{1}}\right)$, which proves (3.12). The last assertion follows by taking $\left(\theta_{1}, \theta_{2}\right)=(1, \theta)$ in (3.12).

REMARK 3.4. If $\theta=n \in \mathbf{N}$, then the definition of $(-A)^{n}$ through (3.6) coincides with that in (2.2), with $A$ being replaced by $-A$. In fact, letting $\left((-A)^{0}, \mathscr{D}\left((-A)^{0}\right)\right)=(I, X)$, from (3.6) and the semigroup property of the negative integer powers of $-A$ we find:

$$
\left\{\begin{aligned}
\mathscr{D}\left((-A)^{n}\right) & =\mathscr{R}\left((-A)^{-n}\right)=\left\{x \in X: x=(-A)^{-(n-1)}(-A)^{-1} y, y \in X\right\} \\
& =\left\{x \in X: x=(-A)^{-(n-1)} f, \text { for some } f \in \mathscr{D}(-A)\right\}, \\
& =\left\{x \in \mathscr{D}\left((-A)^{n-1}\right): \exists f \in \mathscr{D}(-A) \text { such that } f \in(-A)^{n-1} x\right\} \\
& =\left\{x \in \mathscr{D}\left((-A)^{n-1}\right): \mathscr{D}(-A) \cap(-A)^{n-1} x \neq \varnothing\right\}, \\
(-A)^{n} x= & \left\{y \in X: x=(-A)^{-(n-1)}(-A)^{-1} y\right\} \\
= & \left\{y \in X: y \in(-A)\left((-A)^{(n-1)} x\right)\right\} \\
= & (-A)\left((-A)^{n-1} x\right)=\left((-A)(-A)^{n-1}\right) x, \quad x \in \mathscr{D}\left((-A)^{n}\right) .
\end{aligned}\right.
$$

Remarks 3.5-3.8 below explain why, in defining fractional powers for $\mathrm{m}$. 1 . operators, we are prevented to proceed as in the known literature for singlevalued operators.

REMARK 3.5. The main problem of definition (3.6) is that does not provide us of any explicit representation of the fractional powers $(-A)^{\theta}$, $\Re$ e $\theta>1-\beta$. On the other hand, when a $A$ is really a m. 1. operators, the choice of definition (3.6) is motivated by a substantial difference existing between the single- and the multivalued case. That is, the so-called "method of closed extension" used for defining fractional powers of single-valued linear operators, does not work for m. 1. operators. Precisely, let $A$ be a single-valued linear operator and denote by $R_{n}^{+}(\lambda), n \in \mathbf{N} \cup\{0\}, \lambda \in \rho(A)$, the operator $(-1)^{n} \lambda^{-n-1} A(\lambda I-A)^{-1}(-A)^{n}$ having domain $\mathscr{D}\left((-A)^{n}\right)$. According to [23, p. 291], for $\sigma=n+v, n \in \mathbf{N} \cup\{0\}, v \in[0,1)$, define the space $\mathscr{D}^{\sigma}$ as follows:

$$
\mathscr{D}^{\sigma}=\left\{\begin{array}{l}
\left\{x \in \mathscr{D}\left((-A)^{n}\right):(-A)^{n} x \in \overline{\mathscr{D}(A)}\right\}, \text { if } v=0, \\
\left\{x \in \mathscr{D}\left((-A)^{n}\right): R_{n}^{+}(\lambda) x=O\left(\lambda^{-\sigma-1}\right) \text { as } \lambda \rightarrow \infty\right\}, \quad \text { if } v>0 .
\end{array}\right.
$$


Then, in [23], the fractional powers $(-A)^{\zeta}, \Re \mathrm{e} \zeta>0$, of a single-valued linear operator $A$ satisfying (H1) with $\beta=1$ are defined by:

$$
(-A)^{\zeta}=\text { closure of }(-A)_{\sigma}^{\zeta}, \quad \mathscr{D}\left((-A)_{\sigma}^{\zeta}\right)=\mathscr{D}^{\sigma}, \quad \text { if } \Re \mathrm{e} \zeta \in(0, \sigma), \sigma>0,
$$

and, for given $\zeta$, it is shown that the closure is independent on $\sigma>\Re$ e $\zeta$. Here:

$$
(-A)_{\sigma}^{\zeta} x=\left\{\begin{aligned}
&(-A)^{\zeta} x, \text { if } \zeta \in \mathbf{N}, \\
&-\frac{\sin (\zeta \pi)}{\pi}\left[\int_{0}^{N} s^{\zeta}(s I-A)^{-1} x \mathrm{~d} s+\sum_{k=0}^{n}(-1)^{k+1} \frac{N^{\zeta-k}}{\zeta-k}(-A)^{k} x\right. \\
&\left.+\int_{N}^{\infty} s^{\zeta} R_{n}^{+}(s) x \mathrm{~d} s\right], \quad \text { if } \zeta \notin \mathbf{N},
\end{aligned}\right.
$$

where $N>0$ is an arbitrary fixed number. If $\Re \mathrm{e} \zeta \in(n, \sigma)$, we may let $N \rightarrow 0$ and obtain

$$
(-A)_{\sigma}^{\zeta} x=(-1)^{n+1} \frac{\sin (\zeta \pi)}{\pi} \int_{0}^{\infty} s^{\zeta-n-1} A(s I-A)^{-1}(-A)^{n} x \mathrm{~d} s,
$$

which is the expression used in [4] to define $(-A)^{\zeta}$ for $\Re \mathrm{e} \zeta \in(n, n+1), n \in$ $\mathbf{N} \cup\{0\}$, by taking $\mathscr{D}\left((-A)^{n+1}\right)$ instead of $\mathscr{D}^{\sigma}$ as the domain of $(-A)_{\sigma}^{\zeta}$. As usual, the proof that the operators $(-A)_{\sigma}^{\zeta}$ are closable consists in showing that, if $\left\{x_{k}\right\}_{k \in \mathbf{N}} \subset \mathscr{D}^{\sigma}$ is a sequence converging to zero, then the sequence $\left\{(-A)_{\sigma}^{\zeta} x_{k}\right\}_{k \in \mathbf{N}}$, if convergent, has zero limit, too. Letting $y=\lim _{k \rightarrow \infty}(-A)_{\sigma}^{\zeta} x_{k}$, in [4, Lemma 2.1] and [23, Proposition 4.1] the assertion $y=0$ follows from $y \in \mathscr{N}\left((\lambda I-A)^{-1}\right)$, $\lambda \in \rho(A)$. This is just the argument which fails in the multivalued case. For, if $A$ is a m. 1. operator, then $\mathscr{N}\left((\lambda I-A)^{-1}\right)=A 0 \supsetneq\{0\}$, preventing us to conclude the proof as in [4] and [23].

REMARK 3.6. In [22] the fractional powers of single-valued densely defined linear closed operators satisfying $(\mathrm{H} 1)$ with $\beta=1$ are defined by

$$
\left\{\begin{array}{l}
(-A)^{\theta} x=-\frac{\sin (\theta \pi)}{\pi} \mathrm{s}^{-} \lim _{N \rightarrow \infty} \int_{0}^{N} s^{\theta-1} A(s I-A)^{-1} x \mathrm{~d} s, \quad \theta \in(0,1), \\
\mathscr{D}\left((-A)^{\theta}\right)=\left\{x \in X:(-A)^{\theta} x \in X\right\},
\end{array}\right.
$$

where "s-lim" denote the strong limit. This method does not require any closure, but it is with difficulty applicable for defining fractional powers of $\mathrm{m}$. 1. operators, due to the presence of the strong limit in (3.17). Moreover, even only for singlevalued operators, it seems a hard task to remove the assumption $\beta=1$ in this approach, due to the large numbers of technical details in which such assumption plays role in [22]. 
Remark 3.7. Let $A$ be single-valued and let $\beta=1$ in (H1). After having defined via (3.14) the fractional powers $(-A)^{\zeta}, \Re \mathrm{e} \zeta>0$ of $-A$, in $[23$, Sections 4 and 5] the cases $\Re \mathrm{e} \zeta<0$ and $\Re \mathrm{e} \zeta=0$ are considered. Let first $\Re \mathrm{e} \zeta<0$. In [23, Section 5] it is shown that for every $\zeta \in \mathbf{C}$ such that $\Re \mathrm{e} \zeta \in(-(m+1), 0), m \in \mathbf{N}$, the fractional power $(-A)^{\zeta}$ is the operator defined by $(3.5)$ with $\theta=-\zeta$. The definition is then completed by setting

$$
(-A)^{\zeta}=\left(-A^{-1}\right)^{n}, \quad \mathscr{D}\left((-A)^{\zeta}\right)=X, \quad \text { if } \zeta=-n, n \in \mathbf{N},
$$

which is just what follows from (3.1) with $\theta=n$. Hence, due to Remark 3.1, for single-valued linear operators satisfying (H1) with $\beta=1$, our definition (3.1) of the negative fractional powers is equivalent to that in [23]. Let now $\Re \mathrm{e} \zeta=0$. The original idea in [23] for this case is to give another definition of fractional powers, by means of the closure of a family of operators defined in a vertical open strip $\Delta_{\sigma, \tau}=\{\zeta \in \mathbf{C}: \Re \mathrm{e} \zeta \in(-\tau, \sigma)\}, \sigma, \tau>0$, containing the imaginary axis. For the clarity's sake, we remind the definition in [23]. Let $R_{n}^{+}(\lambda)$ be as in Remark 3.5 and let $R_{m}^{-}(\lambda)$ be the operator $(-1)^{m} \lambda^{m}(\lambda I-A)^{-1}(-A)^{-m}, m \in \mathbf{N} \cup\{0\}, \lambda \in \rho(A)$, having domain the whole of $X$. Now, let $\sigma=n+v_{1}$ and $\tau=m+v_{2}$ be positive numbers, where $n, m \in \mathbf{N} \cup\{0\}$ and $v_{j} \in[0,1), j=1,2$. According to [23, formula (4.11)], define the operator $(-A)_{\sigma \tau}^{\zeta}$ with domain $\mathscr{D}^{\sigma}$ by

$$
(-A)_{\sigma, \tau}^{\zeta} x=\left\{\begin{aligned}
x, & \text { if } \zeta=0, \\
(-A)^{\zeta} x, & \text { if } \zeta \in \Delta_{\sigma, \tau} \cap \mathbf{Z}, \\
-\frac{\sin (\zeta \pi)}{\pi}\left[\int_{0}^{N} s^{\zeta} R_{m}^{-}(s) x \mathrm{~d} s+\sum_{k=-m}^{n}(-1)^{k+1} \frac{N^{\zeta-k}}{\zeta-k}\left(-\left.A\right|_{\mathscr{D}^{1}}\right)^{k} x\right. & \\
& \left.+\int_{N}^{\infty} s^{\zeta} R_{n}^{+}(s) x \mathrm{~d} s\right], \quad \text { if } \zeta \in \Delta_{\sigma, \tau} \backslash(\mathbf{Z} \cup\{0\}),
\end{aligned}\right.
$$

where $N>0$ is an arbitrary fixed number. It is show in [23, Proposition 4.11] that $(-A)_{\sigma, \tau}^{\zeta}$ is closable and its closure is independent of $\sigma$ and $\tau$ such that $\zeta \in \Delta_{\sigma, \tau}$. This closure is denoted by $(-A)_{0}^{\zeta}$. When $\Re \mathrm{e} \zeta \in(0, \sigma)$, we may take $m=0$ and $(-A)_{\sigma, \tau}^{\zeta}$ coincides with $(-A)_{\sigma}^{\zeta}$ defined by (3.15). Correspondingly, for $\Re \mathrm{e} \zeta \in(-\tau, 0)$, we may take $n=0$ and $(-A)_{\sigma, \tau}^{\zeta}$ coincides with the restriction to $\mathscr{D}^{\sigma}$ of $(-A)^{\zeta}$ defined by (3.5) with $\theta=-\zeta$ and (3.18). Passing to the closure, we get (cf. [23, pp. 305, 306]) $(-A)_{0}^{\zeta}=(-A)^{\zeta}$ for $\Re \mathrm{e} \zeta>0$ and $(-A)_{0}^{\zeta}=(-A)^{\zeta} \mid \overline{\mathscr{D}(A)}$ for $\Re \mathrm{e} \zeta<0$. In particular (cf. [23, Corollary 5.3]), $(-A)^{\zeta}$, $\Re \mathrm{e} \zeta>0$, defined through (3.14) coincides with $\left((-A)^{-\zeta} \mid \frac{}{\mathscr{D}(A)}\right)^{-1}$, so that $(-A)^{\zeta}(-A)^{-\zeta}=I$ only on $\overline{\mathscr{D}(A)}$. Recalling the observation after formula (3.9), this is less than what we 
obtain by defining $(-A)^{\zeta}, \Re \mathrm{e} \zeta>0$, through (3.6) taking advantage from the injectivity of $(-A)^{-\zeta}$, which, in the single-valued case, comes from [26, p. 285].

REMARK 3.8. Let now $\beta \in(0,1)$ in (H1). In this case, even restricting our interest to single-valued operators, we can not repeat the construction in [23] in order to provide a definition of $(-A)^{ \pm \zeta}$ for every $\zeta \in \mathbf{C}$ which coincides with (3.1) and (3.6) for $\zeta \notin \Delta_{1-\beta}=\{z \in \mathbf{C}: \Re \mathrm{e} z \in[0,1-\beta]\}$. Indeed, if we try to define $(-A)_{0}^{\zeta}$ as in Remark 3.7, the first problem we encounter is that the spaces $\mathscr{D}^{\sigma}$ defined by (3.13) are no longer the suitable ones for the case $\beta \in(0,1)$ in (H1). Maybe, since $R_{0}^{+}(\lambda) x=\lambda^{-1} A(\lambda I-A)^{-1} x=(\lambda I-A)^{-1} x-\lambda^{-1} x=O\left(|\lambda|^{-\beta}\right)$ as $|\lambda| \rightarrow \infty$, a possible different choice could be that of replacing $O\left(\lambda^{-\sigma-1}\right)$ with $O\left(\lambda^{-\sigma-\beta}\right)$ in the second of (3.13). On the other side, the proof of [23, Theorem 2.1], from which the first characterization in (3.13) comes out, is not adaptable to the case $\beta \in(0,1)$, since in that proof the value $\beta=1$ is necessary to ensure that the operator $\lambda R_{0}^{+}(\lambda)$ is uniformly bounded allowing to apply the BanachSteinhaus theorem. Another problem is that we have to take $\sigma$ and $\tau$ greater than $1-\beta$ for comparing the eventual definition of $(-A)_{0}^{ \pm \zeta}$ with that we already know from (3.1) and (3.6) for $\Re \mathrm{e} \zeta>1-\beta$. Also, contrarily to the case $\beta=1$, in which one may take help from formula (3.5) for the construction of $(-A)_{0}^{\zeta}$ for $\Re$ e $\zeta<0$, when $\beta \in(0,1)$ one has only formula $(3.5)$ for $\Re \mathrm{e} \zeta \in(-1, \beta-1)$. Of course, still worst is the case in which $\beta \in(0,1]$ and $A$ is really a m. 1 . operator. In fact, in this case we have to define $(-A)_{0}^{ \pm \zeta}$ so that, when $\Re \mathrm{e} \zeta>1-\beta,(-A)_{0}^{-\zeta}$ and $(-A)_{0}^{\zeta}$ coincide, respectively, with the single-valued bounded linear operator (3.1) and with the closed m. 1 . operator (3.6), but we do not know if $(-A)_{0}^{ \pm \zeta}$ has to be single-valued, multivalued, or of both types in the strip $\Delta_{1-\beta}$.

Now, let $\left\{\mathrm{e}^{t A}\right\}_{t \geq 0} \subset \mathscr{L}(X)$ be the semigroup generated by $A$ and defined by $\mathrm{e}^{0 A}=I$ and the Dunford integrals (2.10). We set

$$
\left[(-A)^{\theta}\right]^{\circ} \mathrm{e}^{t A}=\frac{1}{2 \pi i} \int_{\Gamma}(-\lambda)^{\theta} \mathrm{e}^{t \lambda}(\lambda I-A)^{-1} \mathrm{~d} \lambda, \quad \Re \mathrm{e} \theta \geq 0, t>0,
$$

but here $\left[(-A)^{\theta}\right]^{\circ}$ does not denote a section of $(-A)^{\theta}$ itself. Clearly,

$$
A 0 \subseteq \bigcap_{t>0} \mathscr{N}\left(\left[(-A)^{\theta}\right]^{\circ} \mathrm{e}^{t A}\right), \quad \Re \mathrm{e} \theta \geq 0 .
$$

We recall that operators (3.19) were introduced first in [11] and [12] only for real nonnegative values of $\theta$. However, due to (3.2), it is clear that all the estimates obtained in that papers for $\theta \geq 0$ hold the same for complex $\theta$ such that $\Re$ e $\theta \geq 0$, provided that all the constants depending by $\theta$ in [11] and [12] are rescaled by a factor $\mathrm{e}^{(\pi / 2)|\Im \mathrm{m} \theta|}$. 
Of course, from (2.10) we have $\left[(-A)^{0}\right]^{\circ} \mathrm{e}^{t A}=\mathrm{e}^{t A}, t>0$. Moreover, from (3.1) and (3.19), using the resolvent equation and the residue theorem, we easily derive

$$
\left[(-A)^{1}\right]^{\circ} \mathrm{e}^{t A}(-A)^{-\theta}=\left[(-A)^{1-\theta}\right]^{\circ} \mathrm{e}^{t A}, \quad \Re \mathrm{e} \theta \in(1-\beta, 1], t>0 .
$$

As remarked in $\left[13\right.$, p. 426], if $\Re \mathrm{e} \theta>1-\beta$, the operator $\left[(-A)^{\theta}\right]^{\circ} \mathrm{e}^{t A}$ is really a section of the $\mathrm{m}$. 1. operator $(-A)^{\theta} \mathrm{e}^{t A}$, so that from $(2.11)$ we get

$$
(-1)^{k} D_{t}^{k} \mathrm{e}^{t A}=\left[(-A)^{k}\right]^{\circ} \mathrm{e}^{t A} \subset(-A)^{k} \mathrm{e}^{t A}, \quad t>0, k \in \mathbf{N} .
$$

Finally, (H1) implies the following estimates (see [11, Section 3]):

$$
\left\|\left[(-A)^{\theta}\right]^{\circ} \mathrm{e}^{t A}\right\|_{\mathscr{L}(X)} \leq \tilde{c}_{\alpha, \beta, \theta} t^{(\beta-\Re \mathrm{e} \theta-1) / \alpha}, \quad \Re \mathrm{e} \theta \geq 0, t>0,
$$

where the $\tilde{c}_{\alpha, \beta, \theta}$ 's are positive constants depending on $\alpha, \beta$ and $\theta$. Thus, letting $\theta=0$ in (3.23), we see that, if $\beta \in(0,1)$, the function $t \rightarrow \mathrm{e}^{t A}$ is not bounded as $t \rightarrow 0^{+}$and $\mathrm{e}^{t A}$ is not necessarily strongly continuous in the norm of $X$ on the linear subspace $\overline{\mathscr{D}(A)}$. Indeed, as established in [12, Theorem 3.3], if $\Re \mathrm{e} \zeta>1-\beta$ and $\gamma \in(1-\beta, 1)$, then, when $t \rightarrow 0^{+}$, $\mathrm{e}^{t A}$ converges to $I$ in the norm of $X$ on the domains $\mathscr{D}\left((-A)^{\zeta}\right)$ of the fractional powers $(-A)^{\zeta}$ and on the spaces $X_{A}^{\gamma, \infty}$ defined in the next Section 4, whereas the convergence on the whole $X$ holds only with respect to the seminorm $p_{A}(\cdot)=\left\|A^{-1} \cdot\right\|_{X}$. We stress that later, in Section 5, we shall extend [12, Theorem 3.3] showing that, if $\gamma \in(1-\beta, 1)$, then $\mathrm{e}^{t A}$ is strongly continuous in the norm of $X$ not only on $X_{A}^{\gamma, \infty}$, but in fact on the spaces $(X, \mathscr{D}(A))_{\gamma, p}$ and $X_{A}^{\gamma, p}$ for every $p \in[1, \infty]$ (cf. definitions (4.1) and (4.4)).

The following Lemma 3.9 extends [27, Lemma 2.1.6] to $\mathrm{m}$. 1. operators.

Lemma 3.9. Let $\alpha$ and $\beta$ in assumption (H1) be such that $\alpha+\beta>1$. Then

$$
\int_{0}^{\infty} \mathrm{e}^{-z t} \mathrm{e}^{t A} x \mathrm{~d} t=(z I-A)^{-1} x, \quad \forall \Re \mathrm{e} z>0, \forall x \in X .
$$

In addition, $\bigcap_{t>0} \mathscr{N}\left(\mathrm{e}^{t A}\right)=A 0$. Further, if $\alpha=1$, then $\mathscr{N}\left(\mathrm{e}^{t A}\right)=A 0$ for every $t>0$.

Proof. First, if $\alpha+\beta>1$ and $\Re$ e $z>0$, then the integral on the left-hand side of (3.24) is convergent on $X$. In fact, for every $x \in X$, from (3.23) with $\theta=0$ we have

$$
\left\|\int_{0}^{\infty} \mathrm{e}^{-z t} \mathrm{e}^{t A} x \mathrm{~d} t\right\|_{X} \leq \tilde{c}_{\alpha, \beta, 0}\left[\int_{0}^{\infty} t^{(\beta-1) / \alpha} \mathrm{e}^{-t \Re \mathrm{e} z} \mathrm{~d} t\right]\|x\|_{X},
$$


and the transformation $t \Re \mathrm{e} z=s$ in the latter integral leads to

$$
\left\|\int_{0}^{\infty} \mathrm{e}^{-z t} \mathrm{e}^{t A} x \mathrm{~d} t\right\|_{X} \leq \tilde{c}_{\alpha, \beta, 0}(\Re \mathrm{e} z)^{(1-\alpha-\beta) / \alpha} E((\alpha+\beta-1) / \alpha)\|x\|_{X}<\infty,
$$

where $E(\chi), \chi>0$, denotes the Euler's gamma function $\int_{0}^{\infty} s^{\chi-1} \mathrm{e}^{-s} \mathrm{~d} s$. Hence, using (2.10), or, equivalently, (3.19) with $\theta=0$, Fubini's theorem yields

$$
\begin{aligned}
\int_{0}^{\infty} \mathrm{e}^{-z t} \mathrm{e}^{t A} x \mathrm{~d} t & =\frac{1}{2 \pi i} \int_{\Gamma}\left[\int_{0}^{\infty} \mathrm{e}^{(\lambda-z) t} \mathrm{~d} t\right](\lambda I-A)^{-1} x \mathrm{~d} \lambda \\
& =-\frac{1}{2 \pi i} \int_{\Gamma}(\lambda-z)^{-1}(\lambda I-A)^{-1} x \mathrm{~d} \lambda=(z I-A)^{-1} x
\end{aligned}
$$

Here we have used $\int_{\Gamma}(\lambda-z)^{-1}(\lambda I-A)^{-1} x \mathrm{~d} \lambda=-2 \pi i(z I-A)^{-1} x$ which can be verified using the Cauchy's formula for the holomorphic function $\mu \in \rho(A) \rightarrow$ $(\mu I-A)^{-1} \in \mathscr{L}(X)$ and by closing $\Gamma$ on the right by circles of diameter converging to infinity. Clearly, due to $(2.12)$ and $\mathscr{N}\left((\mu I-A)^{-1}\right)=A 0, \mu \in \rho(A)$, if $x \in A 0$, then all the terms in (3.25) are equal to the zero element of $X$. This completes the proof of (3.24). From (3.24) it follows that, if $x \in \bigcap_{t>0} \mathscr{N}\left(\mathrm{e}^{t A}\right)$, then $x \in \mathscr{N}\left((z I-A)^{-1}\right)=A 0$ for every $\Re \mathrm{e} z>0$. Therefore $\bigcap_{t>0} \mathscr{N}\left(\mathrm{e}^{t A}\right) \subseteq A 0$ which, together with (2.12), leads to $\bigcap_{t>0} \mathscr{N}\left(\mathrm{e}^{t A}\right)=A 0$. Finally, if $\alpha=1$, then from [32, Theorem 5.3] it follows that $\mathrm{e}^{t A}$ can be extended to an analytic semigroup in some sector containing the positive real axis. Now, let $x \in \mathscr{N}\left(e^{t_{0} A}\right)$, $t_{0}>0$. First, the semigroup property implies $\mathrm{e}^{t A} x=0$ for every $t \geq t_{0}$. Then, the map $t \in(0, \infty) \rightarrow \mathrm{e}^{t A} \in \mathscr{L}(X)$ being analytic, we have $\mathrm{e}^{t A} x=0$ for every $t \in(0, \infty)$ and from (3.24) we derive $x \in \mathscr{N}\left((z I-A)^{-1}\right)=A 0$. This shows that, if $\alpha=1$, then $\mathscr{N}\left(\mathrm{e}^{t_{0} A}\right) \subseteq A 0$ for every $t_{0}>0$. Combining this inclusion with (2.12) we obtain the assertion $\mathscr{N}\left(\mathrm{e}^{t_{0} A}\right)=A 0$ for every $t_{0}>0$.

\section{The Spaces $(X, \mathscr{D}(A))_{\gamma, p}$ and $X_{A}^{\gamma, p}$}

Let $A$ be a m. 1. operator satisfying assumption (H1). We introduce here the spaces $(X, \mathscr{D}(A))_{\gamma, p}$ and $X_{A}^{\gamma, p}$, and we recall the basic relations existing among such spaces. From now on, $Y$ being a Banach space, we denote by $C((0, \infty) ; Y)$ the set of all continuos functions from $(0, \infty)$ to $Y$, and for a $Y$-valued strongly measurable function $g(\xi), \xi \in(0, \infty)$, we set $\|g(\xi)\|_{L_{q}^{*}(Y)}=\left(\int_{0}^{\infty}\|g(\xi)\|_{Y}^{q} \frac{\mathrm{d} \xi}{\xi}\right)^{1 / q}$, $q \in[1, \infty)$, and $\|g(\xi)\|_{L_{\infty}^{*}(Y)}=\sup _{\xi \in(0, \infty)}\|g(\xi)\|_{Y}$. Let either $p_{0}, p_{1} \in[1, \infty)$ or $p_{0}=p_{1}=\infty$, and for $\gamma \in(0,1)$ define $p^{-1}=(1-\gamma) p_{0}^{-1}+\gamma p_{1}^{-1}$ if $p_{0}, p_{1} \in[1, \infty)$ and $p=\infty$ if $p_{0}=p_{1}=\infty$. We set 


$$
\left\{\begin{aligned}
(X, \mathscr{D}(A))_{\gamma, p}= & \left\{x \in X: x=v_{0}(\xi)+v_{1}(\xi), \xi \in(0, \infty)\right. \\
& v_{0} \in C((0, \infty) ; X), v_{1} \in C((0, \infty) ; \mathscr{D}(A)), \\
& \left.\left\|\xi^{\gamma} v_{0}(\xi)\right\|_{L_{p_{0}}^{*}(X)}+\left\|\xi^{\gamma-1} v_{1}(\xi)\right\|_{L_{p_{1}}^{*}(\mathscr{D}(A))}<\infty\right\} \\
\|x\|_{(X, \mathscr{D}(A))_{\gamma, p}}= & \inf _{v_{0}, v_{1}}\left\{\left\|\xi^{\gamma} v_{0}(\xi)\right\|_{L_{p_{0}}^{*}(X)}+\left\|\xi^{\gamma-1} v_{1}(\xi)\right\|_{L_{p_{1}}^{*}(\mathscr{D}(A))}\right\}
\end{aligned}\right.
$$

where the infimum is taken over all possible representations of $x$ having the specified form and $\mathscr{D}(A)$ is endowed with the norm (2.13). This characterization of the spaces $(X, \mathscr{D}(A))_{\gamma, p}$ is that obtained by the so called "mean-methods", and it is equivalent to that performed by the usual "K-method" (cf. [35, Theorem 1.5.2 and Remark 1.5.2/2]). Then, due to [35, Theorem 1.3.3], for every $\gamma \in(0,1)$ and $p \in[1, \infty]$ the space $(X, \mathscr{D}(A))_{\gamma, p}$ is an exact interpolation space of exponent $\gamma$ with respect to the pair $(X, \mathscr{D}(A))$. Since both the "mean-methods" and the "K-method" belong to the class of real methods for constructing interpolation spaces (cf. [35]), we shall call the spaces $(X, \mathscr{D}(A))_{\gamma, p}, \gamma \in(0,1), p \in[1, \infty]$, the real interpolation spaces between $X$ and $\mathscr{D}(A)$.

Let us list the main properties of the spaces $(X, \mathscr{D}(A))_{\gamma, p}$. First, if $\mathscr{D}(A)=X$, then $(X, X)_{\gamma, p}=X, \gamma \in(0,1), \quad p \in[1, \infty]$, with equivalence of the respective norms. Then, for every $x \in \mathscr{D}(A)$ there exists a positive constant $c_{1}(\gamma, p)$, $\gamma \in(0,1), \quad p \in[1, \infty]$, such that the interpolation inequality $\|x\|_{(X, \mathscr{D}(A))_{\gamma, p}} \leq$ $c_{1}(\gamma, p)\|x\|_{X}^{1-\gamma}\|x\|_{\mathscr{D}(A)}^{\gamma}$ holds. Further, the spaces $(X, \mathscr{D}(A))_{\gamma, p}$ being intermediate spaces between $X$ and $\mathscr{D}(A)$, i.e. $\mathscr{D}(A) \hookrightarrow(X, \mathscr{D}(A))_{\gamma, p} \hookrightarrow X$, for every $\gamma \in(0,1)$ and $p \in[1, \infty]$ there exists a positive constant $c_{2}(\gamma, p)$ such that $\|x\|_{X} \leq$ $c_{2}(\gamma, p)\|x\|_{(X, \mathscr{D}(A))_{\gamma, p}}$ for every $x \in(X, \mathscr{D}(A))_{\gamma, p}$. Finally, for $\gamma \in(0,1)$ and $1 \leq$ $p_{1} \leq p \leq p_{2} \leq \infty$ we have

$$
\mathscr{D}(A) \hookrightarrow(X, \mathscr{D}(A))_{\gamma, p_{1}} \hookrightarrow(X, \mathscr{D}(A))_{\gamma, p} \hookrightarrow(X, \mathscr{D}(A))_{\gamma, p_{2}} \hookrightarrow X,
$$

whereas, for $0<\gamma_{2}<\gamma_{1}<1$,

$$
(X, \mathscr{D}(A))_{\gamma_{1}, \infty} \hookrightarrow(X, \mathscr{D}(A))_{\gamma_{2}, 1} .
$$

In addition, the inclusion $(X, \mathscr{D}(A))_{\gamma, p} \subseteq \overline{\mathscr{D}(A)}$ holds for every $\gamma \in(0,1)$ and $p \in[1, \infty]$. Then, if $\mathscr{D}(A)$ is closed in $X, \mathscr{D}(A)$ and $(X, \mathscr{D}(A))_{\gamma, p}$ coincide as linear subspaces of $X$ for every $\gamma \in(0,1)$ and $p \in[1, \infty]$, but the norms $\|\cdot\|_{\mathscr{D}(A)}$ and $\|\cdot\|_{(X, \mathscr{D}(A))_{\gamma, p}}$ may be not equivalent, unless that the embedding $(X, \mathscr{D}(A))_{\gamma, p} \hookrightarrow$ $\mathscr{D}(A)$ is shown to hold, too.

For $\gamma \in(0,1)$ and $p \in[1, \infty]$ we now define the Banach spaces $X_{A}^{\gamma, p}$ by

$$
\left\{\begin{array}{l}
X_{A}^{\gamma, p}=\left\{x \in X:[x]_{X_{A}^{\gamma, p}}:=\left\|\xi^{\gamma} A^{\circ}(\xi I-A)^{-1} x\right\|_{L_{p}^{*}(X)}<\infty\right\}, \\
\|x\|_{X_{A}^{\gamma, p}}=\|x\|_{X}+[x]_{X_{A}^{\gamma, p}} .
\end{array}\right.
$$


We recall that, if $A$ is single-valued and $\beta=1$ in (H1), then the classes $(X, \mathscr{D}(A))_{\gamma, p}$ and $X_{A}^{\gamma, p}$ coincide with equivalence of the respective norms (cf. [18, Theorem 3.1], [24, Theorem 3.1] and [35, Theorem 1.14.2]). On the contrary, if $\beta \in(0,1)$, such an equivalence is no longer true, as first observed in [38, Theorem 2] for single-valued operators and, in the case $p=\infty$, in [12, Theorem 1.12] for multivalued ones. Thus, in general, the spaces $X_{A}^{\gamma, p}$ are neither intermediate nor interpolation spaces between $X$ and $\mathscr{D}(A)$.

Remark 4.1. When $A$ is a single-valued densely defined linear operator satisfying (H1) with $\beta=1$, the spaces $X_{A}^{\gamma, 1}$, or, equivalently, $(X, \mathscr{D}(A))_{\gamma, 1}$, play a key role in the definition of the fractional powers of $-A$. To see this, we first observe that, if $\sigma \in(0,1)$, then $\mathscr{D}^{\sigma}$ defined by the second of (3.13) is contained in $X_{A}^{\gamma, 1}$ for every $\gamma \in(0, \sigma)$. In fact, if $x \in \mathscr{D}^{\sigma}$, then $R_{0}^{+}(\lambda) x=\lambda^{-1} A(\lambda I-A)^{-1} x=$ $O\left(\lambda^{-\sigma-1}\right)$ as $\lambda \rightarrow \infty$. Moreover, since $A(\lambda I-A)^{-1}=\lambda(\lambda I-A)^{-1}+I$, from (H1) with $\beta=1$ we deduce $\left\|A(\lambda I-A)^{-1}\right\|_{\mathscr{L}(X)} \leq C+1$. Therefore

$$
[x]_{X_{A}^{\gamma, 1}}=\int_{0}^{\infty} \xi^{\gamma-1}\left\|A(\xi I-A)^{-1} x\right\|_{X} \mathrm{~d} \xi<\infty, \quad \forall x \in \mathscr{D}^{\sigma}, \gamma \in(0, \sigma),
$$

for the integrand goes as $\xi^{\gamma-1}$ at the origin and as $\xi^{\gamma-\sigma-1}$ at infinity. In particular, $\mathscr{D}^{\sigma}$ coincides with $X_{A}^{\sigma, \infty}$. For, if $x \in \mathscr{D}^{\sigma}$, then $[x]_{X_{A}^{\sigma, \infty}}<\infty$, whereas, if $x \in X_{A}^{\sigma, \infty}$, then $R_{0}^{+}(\lambda) x=O\left(\lambda^{-\sigma-1}\right)$. Hence, according to (4.3) in the case $\beta=1$, we have $\mathscr{D}^{\sigma}=X_{A}^{\sigma, \infty} \hookrightarrow X_{A}^{\gamma, 1}$ for every $\gamma \in(0, \sigma)$. We then replace (3.14) with the following

$$
(-A)^{\zeta}=\text { closure of }(-A)_{\sigma}^{\zeta}, \quad \mathscr{D}\left((-A)_{\sigma}^{\zeta}\right)=X_{A}^{\sigma, \infty}, \quad \Re \mathrm{e} \zeta \in(0, \sigma), \sigma \in(0,1),
$$

where, letting $N$ to 0 in $(3.15),(-A)_{\sigma}^{\zeta}$ is defined by (3.16) with $n=0$, that is

$$
(-A)_{\sigma}^{\zeta} x=-\frac{\sin (\zeta \pi)}{\pi} \int_{0}^{\infty} s^{\zeta-1} A(s I-A)^{-1} x \mathrm{~d} s, \quad \Re \mathrm{e} \zeta \in(0, \sigma) .
$$

Clearly, when $\Re \mathrm{e} \zeta \in(0, \sigma)$ the integral on the right-hand side of (4.5) is convergent even for $x$ belonging to the larger space $X_{A}^{\Re \mathrm{e} \zeta, 1}$. The value $\Re \mathrm{e} \zeta=\sigma$ may be considered as well, provided to define $\mathscr{D}\left((-A)_{\sigma}^{\zeta}\right)=X_{A}^{\sigma, 1}$ for $\Re \mathrm{e} \zeta=\sigma$. These observations lead to the following definition of the fractional powers which is the one employed in [24] and [35]:

$$
(-A)^{\zeta}=\text { closure of }(-A)_{\sigma}^{\zeta}, \quad \mathscr{D}\left((-A)_{\sigma}^{\zeta}\right)=X_{A}^{\sigma, 1}, \quad \Re \mathrm{e} \zeta \in(0, \sigma], \sigma \in(0,1),
$$

where $(-A)_{\sigma}^{\zeta}$ is defined by $(4.5)$ and $\mathscr{D}\left((-A)_{\sigma}^{\zeta}\right)$ it is endowed with the graph norm. It is shown in [24, Section 2] that definition (4.6) coincides with that given 
in [23] through (3.14). Observe also that, since $\mathscr{D}^{1}=\mathscr{D}(A)$ due to $\overline{\mathscr{D}(A)}=X$, taking $\sigma=1$ in (3.14) we obtain the definition of $(-A)^{\zeta}, \Re \mathrm{e} \zeta \in(0,1)$, in the sense of [4]:

$$
(-A)^{\zeta}=\text { closure of }(-A)_{1}^{\zeta}, \quad \mathscr{D}\left((-A)_{1}^{\zeta}\right)=\mathscr{D}(A), \quad \Re \mathrm{e} \zeta \in(0,1),
$$

$(-A)_{1}^{\zeta}$ being defined by (4.5). It is clear that (4.7) is equivalent to (4.6), since for every fixed $\zeta$ such that $\Re \mathrm{e} \zeta \in(0,1)$ the domain $\mathscr{D}(A)$ of $(-A)_{1}^{\zeta}$ may be extended to $X_{A}^{\Re \mathrm{e} \zeta, 1}$.

REMARK 4.2. We stress that $\left[X_{A}^{\gamma, p} \cap A 0\right]=\{0\}$ for every $\gamma \in(0,1)$ and $p \in[1, \infty]$. Indeed, assume there exists $x \neq 0$ such that $x \in\left[X_{A}^{\gamma, p} \cap A 0\right]$ for some $\gamma \in(0,1)$ and $p \in[1, \infty]$. Then, since $x \in A 0$, we have $A^{\circ}(\xi I-A)^{-1} x=$ $\xi(\xi I-A)^{-1} x-x=-x$ for every $\xi \in \rho(A)$ and, consequently, $[x]_{X_{A}^{\gamma, p}}=$ $\left\|\xi^{\gamma}\right\|_{L_{p}^{*}(X)}\|x\|_{X}=\infty$, contradicting $x \in X_{A}^{\gamma, p}$.

The following Proposition 4.3 provides a proof of the embeddings announced in $[8$, p. 254], and extends [38] to m. 1 . operators and [12] to $p \in[1, \infty]$.

Proposition 4.3. Let $A$ be a m. l. operator satisfying assumption (H1). Then

$$
\begin{aligned}
& X_{A}^{\gamma, p} \hookrightarrow(X, \mathscr{D}(A))_{\gamma, p}, \quad \gamma \in(0,1), p \in[1, \infty] \\
& (X, \mathscr{D}(A))_{\gamma, p} \hookrightarrow X_{A}^{\gamma+\beta-1, p}, \quad \gamma \in(1-\beta, 1), p \in[1, \infty] .
\end{aligned}
$$

Proof. We begin by showing (4.8). Let $x \in X_{A}^{\gamma, p}, \gamma \in(0,1), p \in[1, \infty]$, and, using $A^{\circ}(\xi I-A)^{-1}=\xi(\xi I-A)^{-1}-I, \xi \in(0, \infty) \subset \Sigma_{\alpha}$, rewrite $x$ in the following form

$$
x=v_{0}(\xi)+v_{1}(\xi), \quad \xi \in(0, \infty)
$$

where $v_{0}(\xi)=-A^{\circ}(\xi I-A)^{-1} x \quad$ and $\quad v_{1}(\xi)=\xi(\xi I-A)^{-1} x$. We have $v_{0} \in$ $C((0, \infty) ; X)$ and $v_{1} \in C((0, \infty) ; \mathscr{D}(A))$. Indeed, let $\xi_{j} \in(0, \infty), j=1,2$. Then, using the resolvent equation and $(\mathrm{H} 1)$, it follows that

$$
\begin{aligned}
& \left\|v_{0}\left(\xi_{1}\right)-v_{0}\left(\xi_{2}\right)\right\|_{X} \\
& =\left\|-\xi_{1}\left(\xi_{1} I-A\right)^{-1} x+\xi_{2}\left(\xi_{2} I-A\right)^{-1} x\right\|_{X} \\
& =\left\|\left(\xi_{2}-\xi_{1}\right)\left(\xi_{1} I-A\right)^{-1} x+\xi_{2}\left[\left(\xi_{2} I-A\right)^{-1}-\left(\xi_{1} I-A\right)^{-1}\right] x\right\|_{X}
\end{aligned}
$$




$$
\begin{aligned}
& =\left\|\left(\xi_{2}-\xi_{1}\right)\left(\xi_{1} I-A\right)^{-1} x+\xi_{2}\left(\xi_{1}-\xi_{2}\right)\left(\xi_{2} I-A\right)^{-1}\left(\xi_{1} I-A\right)^{-1} x\right\|_{X} \\
& \leq C\left|\xi_{1}-\xi_{2}\right|\left(\xi_{1}+1\right)^{-\beta}\|x\|_{X}+C \xi_{2}\left|\xi_{1}-\xi_{2}\right|\left(\xi_{2}+1\right)^{-\beta}\left(\xi_{1}+1\right)^{-\beta}\|x\|_{X} \\
& \leq C\left|\xi_{1}-\xi_{2}\right|\left(\xi_{1}+1\right)^{-\beta}\left[1+C \xi_{2}\left(\xi_{2}+1\right)^{-\beta}\right]\|x\|_{X_{A}^{\gamma, p}} .
\end{aligned}
$$

This shows $v_{0} \in C((0, \infty) ; X)$. Concerning $v_{1}$, instead, we first observe that, since (2.3) implies $A^{\circ}(z I-A)^{-1} \subset A(z I-A)^{-1}, z \in \rho(A)$, the definition of $\|\cdot\|_{\mathscr{D}(A)}$ leads to

$$
\left\|(z I-A)^{-1} y\right\|_{\mathscr{D}(A)} \leq\left\|A^{\circ}(z I-A)^{-1} y\right\|_{X}, \quad \forall y \in X, \forall z \in \rho(A) .
$$

But, for every $z \in \Sigma_{\alpha}$, we have

$$
\begin{aligned}
\left\|A^{\circ}(z I-A)^{-1} y\right\|_{X} & =\left\|\left[z(z I-A)^{-1}-I\right] y\right\|_{X} \\
& \leq\left[C|z|(|z|+1)^{-\beta}+1\right]\|y\|_{X} \\
& \leq(C+1)(|z|+1)^{1-\beta}\|y\|_{X}, \quad \forall y \in X,
\end{aligned}
$$

so that from $(4.11)$ it follows that

$$
\left\|(z I-A)^{-1} y\right\|_{\mathscr{D}(A)} \leq(C+1)(|z|+1)^{1-\beta}\|y\|_{X}, \quad \forall y \in X, \forall z \in \Sigma_{\alpha}
$$

Now, using the resolvent equation,

$$
\begin{aligned}
& \left\|v_{1}\left(\xi_{1}\right)-v_{1}\left(\xi_{2}\right)\right\|_{\mathscr{D}(A)} \\
& \quad=\left\|\xi_{1}\left(\xi_{1} I-A\right)^{-1} x-\xi_{2}\left(\xi_{2} I-A\right)^{-1} x\right\|_{\mathscr{D}(A)} \\
& \quad=\left\|\left(\xi_{1}-\xi_{2}\right)\left(\xi_{1} I-A\right)^{-1} x+\xi_{2}\left[\left(\xi_{1} I-A\right)^{-1}-\left(\xi_{2} I-A\right)^{-1}\right] x\right\|_{\mathscr{D}(A)} \\
& \quad=\left\|\left(\xi_{1}-\xi_{2}\right)\left(\xi_{1} I-A\right)^{-1} x+\xi_{2}\left(\xi_{2}-\xi_{1}\right)\left(\xi_{1} I-A\right)^{-1}\left(\xi_{2} I-A\right)^{-1} x\right\|_{\mathscr{D}(A)} \\
& \quad \leq\left|\xi_{1}-\xi_{2}\right|\left[\left\|\left(\xi_{1} I-A\right)^{-1} x\right\|_{\mathscr{D}(A)}+\xi_{2}\left\|\left(\xi_{1} I-A\right)^{-1}\left(\xi_{2} I-A\right)^{-1} x\right\|_{\mathscr{D}(A)}\right]
\end{aligned}
$$

Hence, applying (4.13) once with $(z, y)=\left(\xi_{1}, x\right)$ and once with $(z, y)=$ $\left(\xi_{1},\left(\xi_{2} I-A\right)^{-1} x\right)$, from $(4.14)$ we deduce

$$
\begin{aligned}
\left\|v_{1}\left(\xi_{1}\right)-v_{1}\left(\xi_{2}\right)\right\|_{\mathscr{D}(A)} & \leq(C+1)\left|\xi_{1}-\xi_{2}\right|\left(\xi_{1}+1\right)^{1-\beta}\left[\|x\|_{X}+\xi_{2}\left\|\left(\xi_{2} I-A\right)^{-1} x\right\|_{X}\right] \\
& \leq(C+1)\left|\xi_{1}-\xi_{2}\right|\left(\xi_{1}+1\right)^{1-\beta}\left[1+C \xi_{2}\left(\xi_{2}+1\right)^{-\beta}\right]\|x\|_{X_{A}^{\gamma, p}}
\end{aligned}
$$


completing the proof of $v_{1} \in C((0, \infty) ; \mathscr{D}(A))$. Therefore, recalling the definition (4.1) of the norm $\|\cdot\|_{(X, \mathscr{D}(A))_{\gamma, p}}$ with $p_{0}=p_{1}=p, p \in[1, \infty]$, from (4.10) and (4.11) we get

$$
\begin{aligned}
\|x\|_{(X, \mathscr{D}(A))_{\gamma, p}} & \leq\left\|\xi^{\gamma} v_{0}(\xi)\right\|_{L_{p}^{*}(X)}+\left\|\xi^{\gamma-1} v_{1}(\xi)\right\|_{L_{p}^{*}(\mathscr{D}(A))} \\
& \leq\left\|\xi^{\gamma} A^{\circ}(\xi I-A)^{-1} x\right\|_{L_{p}^{*}(X)}+\left\|\xi^{\gamma}(\xi I-A)^{-1} x\right\|_{L_{p}^{*}(\mathscr{D}(A))} \\
& \leq 2\left\|\xi^{\gamma} A^{\circ}(\xi I-A)^{-1} x\right\|_{L_{p}^{*}(X)} \\
& \leq 2\|x\|_{X_{A}^{\gamma, p}}, \quad \gamma \in(0,1), p \in[1, \infty] .
\end{aligned}
$$

This completes the proof of (4.8). Let us now prove (4.9). To this purpose, we first notice that when $y \in \mathscr{D}(A)$ from $(2.3)$ we have $A^{\circ}(z I-A)^{-1} y=(z I-A)^{-1} f$, $z \in \rho(A)$, with any $f \in A y$. Then, for $z \in \Sigma_{\alpha}$, from (H1) it follows that $\left\|A^{\circ}(z I-A)^{-1} y\right\|_{X} \leq C(|z|+1)^{-\beta}\|f\|_{X}$ with any $f \in A y$. Taking the infimum with respect to $f \in A y$, we thus find $\left\|A^{\circ}(z I-A)^{-1} y\right\|_{X} \leq C(|z|+1)^{-\beta}\|y\|_{\mathscr{D}(A)}$ for every $y \in \mathscr{D}(A)$, or, equivalently,

$$
\left\|A^{\circ}(z I-A)^{-1}\right\|_{\mathscr{L}(\mathscr{D}(A) ; X)} \leq C(|z|+1)^{-\beta}, \quad \forall z \in \Sigma_{\alpha} .
$$

On the other side, (4.12) implies

$$
\left\|A^{\circ}(z I-A)^{-1}\right\|_{\mathscr{L}(X)} \leq(C+1)(|z|+1)^{1-\beta}, \quad \forall z \in \Sigma_{\alpha}
$$

So, using the interpolation inequality in [27, Proposition 1.2.6] with $\left(X_{1}, Y_{1}, X_{2}, Y_{2}\right)=(X, \mathscr{D}(A), X, X)$, from (4.16) and (4.17) we obtain, for every $\gamma \in(0,1)$ and $p \in[1, \infty]$ :

$$
\left\|A^{\circ}(z I-A)^{-1}\right\|_{\mathscr{L}\left((X, \mathscr{D}(A))_{\gamma, p} ; X\right)} \leq c_{3}(\gamma, p)(|z|+1)^{1-\beta-\gamma}, \quad \forall z \in \Sigma_{\alpha} .
$$

Let now $x \in(X, \mathscr{D}(A))_{\gamma, p}, \gamma \in(1-\beta, 1), p \in[1, \infty]$. If $p=\infty$, then from (4.18) we get

$$
\begin{aligned}
{[x]_{X_{A}^{\gamma+\beta-1, \infty}} } & =\left\|\xi^{\gamma+\beta-1} A^{\circ}(\xi I-A)^{-1} x\right\|_{L_{\infty}^{*}(X)} \\
& \leq \sup _{\xi \in(0, \infty)}\left[\xi(\xi+1)^{-1}\right]^{\gamma+\beta-1} c_{3}(\gamma, \infty)\|x\|_{(X ; \mathscr{D}(A))_{\gamma, \infty}}
\end{aligned}
$$

Hence, using $\|x\|_{X} \leq c_{2}(\gamma, p)\|x\|_{(X ; \mathscr{D}(A))_{\gamma, p}}, p \in[1, \infty]$, from the latter inequality we derive

$$
\|x\|_{X_{A}^{\gamma+\beta-1, \infty}} \leq c_{4}(\gamma, \infty)\|x\|_{(X ; \mathscr{D}(A))_{\gamma, \infty}}, \quad \gamma \in(1-\beta, 1),
$$


where $c_{4}(\gamma, \infty)=\sum_{j=2}^{3} c_{j}(\gamma, \infty)$. This proves (4.9) with $p=\infty$. If $p \in[1, \infty)$, then

$$
[x]_{X_{A}^{\gamma+\beta-1, p}}^{p}=\left\|\xi^{\gamma+\beta-1} A^{\circ}(\xi I-A)^{-1} x\right\|_{L_{p}^{*}(X)}^{p}=I_{1}+I_{2}
$$

where

$$
I_{1}=\int_{0}^{1} \xi^{(\gamma+\beta-1) p}\left\|A^{\circ}(\xi I-A)^{-1} x\right\|_{X}^{p} \frac{\mathrm{d} \xi}{\xi}, \quad I_{2}=\int_{1}^{\infty} \xi^{(\gamma+\beta-1) p}\left\|A^{\circ}(\xi I-A)^{-1} x\right\|_{X}^{p} \frac{\mathrm{d} \xi}{\xi} .
$$

Of course, since $1-\beta-\gamma<0$ for $\gamma \in(1-\beta, 1)$, (4.18) leads us to

$$
I_{1} \leq\left[c_{3}(\gamma, p)\right]^{p}\left[\int_{0}^{1} \xi^{(\gamma+\beta-1) p-1} \mathrm{~d} \xi\right]\|x\|_{(X ; \mathscr{D}(A))_{\gamma, p}}^{p}=\left[c_{5}(\beta, \gamma, p)\right]^{p}\|x\|_{(X ; \mathscr{D}(A))_{\gamma, p}}^{p},
$$

where $c_{5}(\beta, \gamma, p)=[(\gamma+\beta-1) p]^{-1 / p} c_{3}(\gamma, p)$. As far as $I_{2}$ is concerned, we write $x=v_{0}(\xi)+v_{1}(\xi), \xi \in(0, \infty)$, the functions $v_{j}, j=0,1$, having the properties in definition (4.1). Then, using $(a+b)^{p} \leq 2^{p-1}\left(a^{p}+b^{p}\right), a, b \geq 0, p \in[1, \infty)$, we have

$$
I_{2} \leq 2^{p-1}\left[I_{2,0}+I_{2,1}\right]
$$

where we have set

$$
I_{2, j}=\int_{1}^{\infty} \xi^{(\gamma+\beta-1) p}\left\|A^{\circ}(\xi I-A)^{-1} v_{j}(\xi)\right\|_{X}^{p} \frac{\mathrm{d} \xi}{\xi}, \quad j=0,1 .
$$

From (4.17) applied to $I_{2,0}$ and $\xi^{-1}(\xi+1) \leq 2, \xi \in[1, \infty)$, it thus follows

$$
\begin{aligned}
I_{2,0} & \leq(C+1)^{p} \int_{1}^{\infty} \xi^{\gamma p}\left[\xi^{-1}(\xi+1)\right]^{(1-\beta) p}\left\|v_{0}(\xi)\right\|_{X}^{p} \frac{\mathrm{d} \xi}{\xi} \\
& \leq\left[c_{6}(\beta)\right]^{p}\left\|\xi^{\gamma} v_{0}(\xi)\right\|_{L_{p}^{*}(X)}^{p},
\end{aligned}
$$

with $c_{6}(\beta)=2^{(1-\beta)}(C+1)$. Instead, from (4.16) applied to $I_{2,1}$, it follows that

$$
I_{2,1} \leq C^{p} \int_{1}^{\infty} \xi^{(\gamma-1) p}\left[\xi(\xi+1)^{-1}\right]^{\beta p}\left\|v_{1}(\xi)\right\|_{\mathscr{D}(A)}^{p} \frac{\mathrm{d} \xi}{\xi} \leq C^{p}\left\|\xi^{\gamma-1} v_{1}(\xi)\right\|_{L_{p}^{*}(\mathscr{D}(A))}^{p} .
$$

Then, taking the infimum over all possible representations of the form $x=$ $v_{0}(\xi)+v_{1}(\xi)$, from $(4.21)-(4.23)$ we obtain

$$
I_{2} \leq\left[c_{7}(\beta, p)\right]^{p}\|x\|_{(X ; \mathscr{D}(A))_{\gamma, p}}^{p},
$$

where $c_{7}(\beta, p)=2^{(p-1) / p} c_{6}(\beta)$. Finally, summing up (4.19), (4.20) and (4.24), we have

$$
[x]_{X_{A}^{\gamma+\beta-1, p}}^{p} \leq\left[c_{8}(\beta, \gamma, p)\right]^{p}\|x\|_{(X ; \mathscr{D}(A))_{\gamma, p}}^{p},
$$


with $c_{8}(\beta, \gamma, p)=\left\{\left[c_{5}(\beta, \gamma, p)\right]^{p}+\left[c_{7}(\beta, p)\right]^{p}\right\}^{1 / p}$. Therefore,

$$
\|x\|_{X_{A}^{\gamma+\beta-1, p}} \leq c_{9}(\beta, \gamma, p)\|x\|_{(X ; \mathscr{D}(A))_{\gamma, p}}, \quad \gamma \in(1-\beta, 1), p \in[1, \infty),
$$

where $c_{9}(\beta, \gamma, p)=c_{2}(\gamma, p)+c_{8}(\beta, \gamma, p)$. The proof is complete.

Remark 4.4. If $A$ is single-valued and $\beta=1$, then the proof of (4.9) may be performed in a easier way. We refer to $[18$, p. 158-160] for the details, but the basic fact is that in the single-valued case one may take advantage from the inequality $\left\|A(z I-A)^{-1} y\right\|_{X} \leq\left\|(z I-A)^{-1} y\right\|_{\mathscr{D}(A)}, z \in \rho(A), y \in X$, which fails in the multivalued case (cf. (4.11)).

Remark 4.5. By setting $\varphi=\gamma+\beta-1, \gamma \in(1-\beta, 1)$, from (4.2) and (4.9) it follows that $\mathscr{D}(A) \hookrightarrow(X, \mathscr{D}(A))_{1+\varphi-\beta, p} \hookrightarrow X_{A}^{\varphi, p} \hookrightarrow X, \varphi \in(0, \beta), p \in[1, \infty]$. Then, according to what we have noticed before Remark 4.1, if $\beta \in(0,1)$, the spaces $X_{A}^{\varphi, p}$ are intermediate spaces between $X$ and $\mathscr{D}(A)$ only for $\varphi \in(0, \beta)$, whereas when $\varphi \in[\beta, 1)$ they may be smaller than $\mathscr{D}(A)$. We mention that the embedding $\mathscr{D}(A) \hookrightarrow X_{A}^{\varphi, p}, \varphi \in(0, \beta), p \in[1, \infty]$, can be shown even using only the definitions of the norms $\|\cdot\|_{\mathscr{D}(A)}$ and $\|\cdot\|_{X_{A}^{\delta, p}}$ and avoiding any argument of interpolation theory (cf. [9, Section 2]).

REMARK 4.6. We can now refine Remark 4.2. Indeed, (4.9) implies $\{0\} \subseteq$ $\left[(X, \mathscr{D}(A))_{\gamma, p} \cap A 0\right] \subseteq\left[X_{A}^{\gamma+\beta-1, p} \cap A 0\right]=\{0\}, \quad \gamma \in(1-\beta, 1), \quad p \in[1, \infty]$, whereas, when $\beta<1,\{0\}$ may be a proper subset of $\left[(X, \mathscr{D}(A))_{\gamma, p} \cap A 0\right], \gamma \in(0,1-\beta]$. In any case $[\mathscr{D}(A) \cap A 0]=\{0\}$, since Remark 4.5 implies $\mathscr{D}(A) \cap A 0 \subseteq X_{A}^{\varphi, p} \cap A 0$ for every $\varphi \in(0, \beta)$ and $p \in[1, \infty]$. On the contrary, due to the inclusion $(X, \mathscr{D}(A))_{\gamma, p} \subseteq \overline{\mathscr{D}(A)}$ for every $\gamma \in(0,1)$ and $p \in[1, \infty]$, in general it is not true that $[\overline{\mathscr{D}}(A) \cap A 0]=\{0\}$. This is true, instead, if $\beta=1$. In this case the topological direct sum $\overline{\mathscr{D}(A)} \oplus A 0$ is a closed subspace of $X$, and, if $X$ is reflexive, it coincides with the whole $X$ (cf. [40, Theorems 2.4 and 2.6]). We refer to [28], for an equivalence between the identity $X=\overline{\mathscr{D}(A)} \oplus A 0$ and the well-posedness on $\mathscr{D}(A)$ of the abstract multivalued Cauchy problem $D_{t} w(t) \in A w(t), t \in(0, T]$, $w(0)=x$.

REMARK 4.7. In particular, if $A$ is a m. 1. operator satisfying (H1) and $\mathscr{D}(A)=X$, then from $[\mathscr{D}(A) \cap A 0]=\{0\}$ it follows that $A 0=\{0\}$ and $A$ is necessarily single-valued.

Observe that, with the exception of the case $\beta=1$ when $(X, \mathscr{D}(A))_{\gamma, p}$ and $X_{A}^{\gamma, p}$ coincide with equivalence of the respective norms, in general it is not clear if 
embeddings analogous to (4.2) and (4.3) hold even for the spaces $X_{A}^{\gamma, p}$. In fact, using (4.2), (4.3), (4.8) and (4.9) we can only prove that, if $\gamma \in(1-\beta, 1)$ and $1 \leq p_{1} \leq p_{2} \leq \infty$, then

$$
X_{A}^{\gamma, p_{1}} \hookrightarrow(X, \mathscr{D}(A))_{\gamma, p_{1}} \hookrightarrow(X, \mathscr{D}(A))_{\gamma, p_{2}} \hookrightarrow X_{A}^{\gamma+\beta-1, p_{2}}
$$

whereas, if $1-\beta<\gamma_{2}<\gamma_{1}<1$, then $X_{A}^{\gamma_{1}, \infty} \hookrightarrow(X, \mathscr{D}(A))_{\gamma_{1}, \infty} \hookrightarrow(X, \mathscr{D}(A))_{\gamma_{2}, 1} \hookrightarrow$ $X_{A}^{\gamma_{2}+\beta-1,1}$. We also recall that, if $A$ is single-valued, densely defined, and satisfies assumption (H1) with $\beta=1$, then the spaces $X_{A}^{\gamma, p}$, or, equivalently, $(X, \mathscr{D}(A))_{\gamma, p}$, are linked to the domains $\mathscr{D}\left((-A)^{\theta}\right)$ of the fractional powers of $-A$ by the following continuous embedding (cf. [24, Proposition 2.8], [25, p. 12] and [35, Theorem 1.15.2(d) with $m=1])$ :

$$
X_{A}^{\Re \mathrm{e} \theta, 1} \hookrightarrow \mathscr{D}\left((-A)^{\theta}\right) \hookrightarrow X_{A}^{\Re \mathrm{e} \theta, \infty}, \quad \Re \mathrm{e} \theta \in(0,1) .
$$

Combining (4.3) with (4.26) we find also $\mathscr{D}\left((-A)^{\theta_{1}}\right) \hookrightarrow \mathscr{D}\left((-A)^{\theta_{2}}\right), 0<\Re$ e $\theta_{2}<$ $\Re$ e $\theta_{1}<1$, which agrees with (3.12) with $\beta=1$. Later on, in Section 6 , we shall generalize (4.26) to multivalued (and hence, possibly single-valued) linear operators satisfying $(\mathrm{H} 1)$ with $\beta \in(0, \alpha], \alpha \in(0,1]$, and having not necessarily dense domain $\mathscr{D}(A)$.

We conclude this section with two open problems which may constitute the subject of future investigations. Indeed, although our result, that is a version of (4.26) for $\mathrm{m}$. 1. operators satisfying (H1), is enough satisfactory from the point of view of applications, especially those dealing with optimal regularity for degenerate differential equations, sometimes it may be useful to have a precise characterization of the domains $\mathscr{D}\left((-A)^{\theta}\right)$. When $A$ is a closed single-valued linear operator having dense domain and satisfying $(\mathrm{H} 1)$ with $\beta=1$, a remarkable result in this direction is the following (cf. [24, Proposition 2.9]): if there exists $\theta_{1} \in \mathbf{C}$ such that $\Re \mathrm{e} \theta_{1} \in(0,1)$ and $\mathscr{D}\left((-A)^{\theta_{1}}\right)$ coincides, in the sense of equivalence of the norms, with $X_{A}^{\Re \mathrm{e} \theta_{1}, p}$ for some $p \in[1, \infty]$, then $\mathscr{D}\left((-A)^{\theta_{2}}\right)=$ $X_{A}^{\Re \mathrm{e} \theta_{2}, p}$ for every $\theta_{2} \in \mathbf{C}$ such that $\Re \mathrm{e} \theta_{2} \in(0,1)$. In particular, $\mathscr{D}\left((-A)^{\theta_{1}}\right)=$ $\mathscr{D}\left((-A)^{\theta_{2}}\right)$ if $\Re \mathrm{e} \theta_{1}=\Re \mathrm{e} \theta_{2}$. Also, in [24], an example and a counterexample of this fact are given. At the moment, even assuming $\beta=1$ in (H1), we do not know if this result can be extended to m. 1. operators. For, in the proof of [24, Proposition 2.9], a basic role is played by the definition of $(-A)^{\theta}$ as the closure of the operator $(-A)_{\sigma}^{\theta}$ defined in (3.15) (cf. [24, Theorems 2.6 and 2.7]), which is just the argument failing in the multivalued case. Further, it is not even known if, and if how, the result in [24] changes for single-valued linear operators when $\beta=1$ in (H1) is replaced by $\beta \in(0, \alpha], \alpha \in(0,1]$. 
In the single-valued case with $\beta=1$ another interesting relation is that provided in [35, Theorem 1.15.3]: assume there exist $\varepsilon, C^{\prime \prime}>0$ such that $\left\|(-A)^{\mathrm{i} t}\right\|_{\mathscr{L}(X)} \leq C^{\prime \prime}$ for every $t \in[-\varepsilon, \varepsilon]$. Then, for every $\Re \mathrm{e} \theta_{1}>\Re \mathrm{e} \theta_{2} \geq 0$ and $\gamma \in(0,1)$ it holds that

$$
\left[\mathscr{D}\left((-A)^{\theta_{2}}\right), \mathscr{D}\left((-A)^{\theta_{1}}\right)\right]_{\gamma}=\mathscr{D}\left((-A)^{(1-\gamma) \theta_{2}+\gamma \theta_{1}}\right) .
$$

Here, $\left[X_{1}, X_{2}\right]_{\delta}, \delta \in(0,1)$, denote the complex interpolation space of exponent $\delta$ between two Banach spaces $X_{1}$ and $X_{2}$ (cf. [35, Sections 1.9]). This result has been successfully applied by several authors in many concrete cases. We quote, for instance, [2, Section 7], [14, Section 1], [15, Section 3], [16, Section 6], and [30, Section 4]. It is therefore clear how much efficient it would be to have an analogous characterization for the domains of fractional powers of $\mathrm{m}$. 1. operators (possibly single-valued) satisfying (H1) with $\beta \in(0,1]$. According to Remark 3.8, the main problem in generalizing this result is the condition on the pure imaginary powers of $-A$, whose meaning, if any, is lacking when $\beta \in(0,1]$ in the multivalued case and when $\beta \in(0,1)$ in the single-valued one. It is in this spirit that should be regarded our attempt of giving, in Section 9, a definition of the fractional powers $(-A)^{ \pm \zeta}$ for $\Re \mathrm{e} \zeta \in[0,1-\beta]$.

\section{Strong Continuity of $\mathrm{e}^{t A}$ on $(X, \mathscr{D}(A))_{\gamma, p}$ and $X_{A}^{\gamma, p}$}

Let $A$ be a m. 1. operator $A$ satisfying assumption (H1). Then the linear operator $\left[(-A)^{1}\right]^{\circ} \mathrm{e}^{t A}$ (cf. (3.19)) satisfies the following estimates (cf. [12, Proposition 3.4]):

$$
\left\|\left[(-A)^{1}\right]^{\circ} \mathrm{e}^{t A}\right\|_{\mathscr{L}\left(X_{A}^{\gamma, \infty} ; X\right)} \leq c_{10} t^{(\beta+\gamma-2) / \alpha}, \quad t>0, \gamma \in(1-\beta, 1),
$$

$c_{10}$ being a positive constant depending on $\alpha, \beta$ and $\gamma$. Due to the strong continuity of $\left\{\mathrm{e}^{t A}\right\}_{t \geq 0}$ in the norm of $X$ on the space $X_{A}^{\gamma, \infty}, \gamma \in(1-\beta, 1)$, proved in [12, Theorem 3.3], for every $x \in X_{A}^{\gamma, \infty}, \gamma \in(1-\beta, 1)$, using (3.22) we can write $\mathrm{e}^{t A} x-x=-\int_{0}^{t}\left[(-A)^{1}\right]^{\circ} \mathrm{e}^{s A} x \mathrm{~d} s$. Therefore (cf. [12, Theorem 3.5]), if $\alpha+\beta>1$, estimate (5.1) easily yields:

$$
\left\|\mathrm{e}^{t A}-I\right\|_{\mathscr{L}\left(X_{A}^{\gamma, \infty} ; X\right)} \leq c_{11} t^{(\alpha+\beta+\gamma-2) / \alpha}, \quad t>0, \gamma \in(2-\alpha-\beta, 1),
$$

$c_{11}$ being a positive constant depending on $\alpha, \beta$ and $\gamma$. We now improve (5.1) and (5.2) showing that in those estimates $X_{A}^{\gamma, \infty}$ can in fact be replaced by $(X, \mathscr{D}(A))_{\gamma, p}$ and $X_{A}^{\gamma, p}, p \in[1, \infty]$. We begin with the following Lemma 5.1 which refines $(5.1)$. 
Lemma 5.1. Let $A$ be a m. l. operator satisfying (H1) and let $Y_{\gamma}^{p} \in$ $\left\{(X, \mathscr{D}(A))_{\gamma, p}, X_{A}^{\gamma, p}\right\}, \gamma \in(0,1), p \in[1, \infty]$. Then, there exists a positive constant $c_{12}=c_{12}(\alpha, \beta, \gamma, p)$ such that:

$$
\left\|\left[(-A)^{1}\right]^{\circ} \mathrm{e}^{t A}\right\|_{\mathscr{L}\left(Y_{\gamma}^{p} ; X\right)} \leq c_{12} t^{(\beta+\gamma-2) / \alpha}, \quad t>0, \gamma \in(0,1), p \in[1, \infty] .
$$

Proof. We first prove $(5.3)$ with $Y_{\gamma}^{p}=(X, \mathscr{D}(A))_{\gamma, p}$ by interpolation. Let $x \in \mathscr{D}(A)$ and $y \in A x$. Then $x=A^{-1} y=(-A)^{-1}(-y)$ and from (3.21) with $\theta=1$ we deduce

$$
\left[(-A)^{1}\right]^{\circ} \mathrm{e}^{t A} x=\left[(-A)^{1}\right]^{\circ} \mathrm{e}^{t A}(-A)^{-1}(-y)=\left[(-A)^{0}\right]^{\circ} \mathrm{e}^{t A}(-y)=-\mathrm{e}^{t A} y .
$$

Hence, (3.23) with $\theta=0$ yields:

$$
\left\|\left[(-A)^{1}\right]^{\circ} \mathrm{e}^{t A} x\right\|_{X} \leq\left\|\mathrm{e}^{t A} y\right\|_{X} \leq \tilde{c}_{\alpha, \beta, 0} t^{(\beta-1) / \alpha}\|y\|_{X}, \quad t>0, y \in A x .
$$

Passing to the infimum with respect to $y \in A x$ in the latter inequality we thus get $\left\|\left[(-A)^{1}\right]^{\circ} \mathrm{e}^{t A} x\right\|_{X} \leq \tilde{c}_{\alpha, \beta, 0} t^{(\beta-1) / \alpha}\|x\|_{\mathscr{D}(A)}, x \in \mathscr{D}(A)$, or, equivalently,

$$
\left\|\left[(-A)^{1}\right]^{\circ} \mathrm{e}^{t A}\right\|_{\mathscr{L}(\mathscr{D}(A) ; X)} \leq \tilde{c}_{\alpha, \beta, 0} t^{(\beta-1) / \alpha}, \quad t>0 .
$$

Furthermore, from (3.23) with $\theta=1$ we have:

$$
\left\|\left[(-A)^{1}\right]^{\circ} \mathrm{e}^{t A}\right\|_{\mathscr{L}(X)} \leq \tilde{c}_{\alpha, \beta, 1} t^{(\beta-2) / \alpha}, \quad t>0 .
$$

Now, using the interpolation inequality [27, Proposition 1.2.6] with $\left(X_{1}, Y_{1}, X_{2}, Y_{2}\right)=(X, \mathscr{D}(A), X, X)$, from (5.4) and (5.5) we finally find, for every $\gamma \in(0,1)$ and $p \in[1, \infty]$ :

$$
\left\|\left[(-A)^{1}\right]^{\circ} \mathrm{e}^{t A}\right\|_{\mathscr{L}\left((X, \mathscr{D}(A))_{\gamma, p} ; X\right)} \leq c_{13} t^{(\beta+\gamma-2) / \alpha}, \quad t>0 .
$$

This proves (5.3) with $Y_{\gamma}^{p}=(X, \mathscr{D}(A))_{\gamma, p}$ and $c_{12}=c_{13}$. To prove (5.3) with $Y_{\gamma}^{p}=X_{A}^{\gamma, p}$ it suffices to recall that, if $X_{j}, j=1,2,3$, are three Banach spaces with $X_{1} \hookrightarrow X_{2}$ and $L \in \mathscr{L}\left(X_{2} ; X_{3}\right)$, then $L \in \mathscr{L}\left(X_{1} ; X_{3}\right)$ and $\|L\|_{\mathscr{L}\left(X_{1} ; X_{3}\right)} \leq$ $c_{0}\|L\|_{\mathscr{L}\left(X_{2} ; X_{3}\right)}$, where $c_{0}>0$ is such that $\|x\|_{X_{2}} \leq c_{0}\|x\|_{X_{1}}$ for every $x \in X_{1}$. Then, the assertion follows from (5.6) and embedding (4.8). In particular (cf. (4.15)), $c_{12}=c_{0} c_{13}=2 c_{13}$.

The following Proposition 5.2 extends [12, Theorem 3.3].

Proposition 5.2. Let $A$ be a m. l. operator satisfying (H1). If $\gamma \in(1-\beta, 1)$, then $\mathrm{e}^{t A}$ is strongly continuous in the norm of $X$ on $(X, \mathscr{D}(A))_{\gamma, p}$ and $X_{A}^{\gamma, p}$ for every $p \in[1, \infty]$. 
Proof. First, let $x \in(X, \mathscr{D}(A))_{\gamma, p}, \gamma \in(1-\beta, 1), p \in[1, \infty]$, and set

$$
w=\frac{1}{2 \pi i} \int_{\Gamma} \lambda^{-1} A^{\circ}(\lambda I-A)^{-1} x \mathrm{~d} \lambda,
$$

where $\Gamma$ is the contour in (2.10). We shall prove that $\lim _{t \rightarrow 0^{+}} \mathrm{e}^{t A} x=w$ and $w-x \in\left[X_{A}^{\gamma+\beta-1, \infty} \cap A 0\right]$, so that from Remark 4.2 it will follows $w=x$. We begin by proving that $w$ is a well defined element of $X$. To see this we observe that, using estimate (4.18), $|\lambda| \geq c>0$ for every $\lambda \in \Gamma$, and $\gamma \in(1-\beta, 1)$, from (5.7) we get

$$
\begin{aligned}
\|w\|_{X} & \leq(2 \pi)^{-1} c_{3}(\gamma, p)\left[\int_{\Gamma}|\lambda|^{-1}(|\lambda|+1)^{1-\beta-\gamma}|\mathrm{d} \lambda|\right]\|x\|_{(X, \mathscr{D}(A))_{\gamma, p}} \\
& \leq \pi^{-1} c_{3}(\gamma, p)\left[\int_{c}^{\infty} \eta^{-1}(\eta+1)^{1-\beta-\gamma} \mathrm{d} \eta\right]\|x\|_{(X, \mathscr{D}(A))_{\gamma, p}} \\
& \leq \pi^{-1} c_{3}(\gamma, p)\left[\int_{c}^{\infty} \eta^{-\beta-\gamma} \mathrm{d} \eta\right]\|x\|_{(X, \mathscr{D}(A))_{\gamma, p}} \\
& =[\pi(\gamma+\beta-1)]^{-1} c^{1-\beta-\gamma} c_{3}(\gamma, p)\|x\|_{(X, \mathscr{D}(A))_{\gamma, p}}
\end{aligned}
$$

Hence $\|w\|_{X}<\infty$, i.e. $w \in X$. Now, we show that $\lim _{t \rightarrow 0^{+}} \mathrm{e}^{t A} x=w$. Due to $(\lambda I-A)^{-1}=\lambda^{-1}\left[A^{\circ}(\lambda I-A)^{-1}+I\right]$ and $\int_{\Gamma} \lambda^{-1} e^{t \lambda} \mathrm{d} \lambda=0, t>0$, we rewrite $(2.10)$ as

$$
\mathrm{e}^{t A} x=\frac{1}{2 \pi i} \int_{\Gamma} \lambda^{-1} \mathrm{e}^{t \lambda} A^{\circ}(\lambda I-A)^{-1} x \mathrm{~d} \lambda, \quad t>0 .
$$

Therefore, exploiting (5.8) and Lebesgue's dominated convergence theorem we derive

$$
\lim _{t \rightarrow 0^{+}} \mathrm{e}^{t A} x=\frac{1}{2 \pi i} \int_{\Gamma} \lim _{t \rightarrow 0^{+}} \lambda^{-1} \mathrm{e}^{t \lambda} A^{\circ}(\lambda I-A)^{-1} x \mathrm{~d} \lambda=w .
$$

Finally, let us prove $w-x \in\left[X_{A}^{\gamma+\beta-1, \infty} \cap A 0\right]=\{0\}$. Using $A^{-1} \in \mathscr{L}(X)$ to commute $A^{-1}$ with the integral sign, from (2.6) and (3.1) with $\theta=1$ we find

$$
A^{-1} w=\frac{1}{2 \pi i} \int_{\Gamma} \lambda^{-1} A^{-1} A^{\circ}(\lambda I-A)^{-1} x \mathrm{~d} \lambda=\frac{1}{2 \pi i} \int_{\Gamma} \lambda^{-1}(\lambda I-A)^{-1} x \mathrm{~d} \lambda=A^{-1} x,
$$

that is $w-x \in \mathscr{N}\left(A^{-1}\right)=A 0$. Moreover, $(2.7)$ and $\int_{\Gamma} \lambda^{-1}(\lambda-\xi)^{-1} \mathrm{~d} \lambda=0, \xi>0$, yield 


$$
\begin{aligned}
(\xi I-A)^{-1} w & =\frac{1}{2 \pi i} \int_{\Gamma} \lambda^{-1}(\xi I-A)^{-1} A^{\circ}(\lambda I-A)^{-1} x \mathrm{~d} \lambda \\
& =\frac{1}{2 \pi i} \int_{\Gamma} \lambda^{-1}(\lambda-\xi)^{-1}\left[A^{\circ}(\xi I-A)^{-1}-A^{\circ}(\lambda I-A)^{-1}\right] x \mathrm{~d} \lambda \\
& =-\frac{1}{2 \pi i} \int_{\Gamma} \lambda^{-1}(\lambda-\xi)^{-1} A^{\circ}(\lambda I-A)^{-1} x \mathrm{~d} \lambda, \quad \xi>0 .
\end{aligned}
$$

Then

$$
\begin{aligned}
A^{\circ}(\xi I-A)^{-1} w & =\xi(\xi I-A)^{-1} w-w \\
& =-\frac{1}{2 \pi i} \int_{\Gamma} \lambda^{-1}\left[\xi(\lambda-\xi)^{-1}+1\right] A^{\circ}(\lambda I-A)^{-1} x \mathrm{~d} \lambda \\
& =-\frac{1}{2 \pi i} \int_{\Gamma}(\lambda-\xi)^{-1} A^{\circ}(\lambda I-A)^{-1} x \mathrm{~d} \lambda, \quad \xi>0 .
\end{aligned}
$$

Observe now that for every $\lambda \in \Gamma$ and $\xi>0$ it holds that

$$
\begin{aligned}
|\lambda-\xi| & =\left\{\left[c(|\eta|+1)^{\alpha}+\xi\right]^{2}+\eta^{2}\right\}^{1 / 2} \\
& \geq\left(\xi^{2}+\eta^{2}\right)^{1 / 2} \geq 2^{-1 / 2}(\xi+|\eta|), \quad \eta \in \mathbf{R} .
\end{aligned}
$$

Using (4.18) and (5.11), from (5.10) it thus follows, for every $\xi>0$ and $\gamma \in(1-\beta, 1)$ :

$$
\begin{aligned}
\left\|A^{\circ}(\xi I-A)^{-1} w\right\|_{X} & \leq(2 \pi)^{-1} c_{3}(\gamma, p)\left[\int_{\Gamma}|\lambda-\xi|^{-1}(|\lambda|+1)^{1-\beta-\gamma}|\mathrm{d} \lambda|\right]\|x\|_{(X, \mathscr{D}(A))_{\gamma, p}} \\
& \leq 2^{1 / 2} \pi^{-1} c_{3}(\gamma, p)\left[\int_{0}^{\infty}(\xi+\eta)^{-1} \eta^{1-\beta-\gamma} \mathrm{d} \eta\right]\|x\|_{(X, \mathscr{D}(A))_{\gamma, p}}
\end{aligned}
$$

Hence, setting $c_{14}=2^{1 / 2} \pi^{-1} c_{3}(\gamma, p)$ and changing the variable from $\eta$ to $\xi \mu$, we get

$$
\left\|A^{\circ}(\xi I-A)^{-1} w\right\|_{X} \leq c_{14} \xi^{1-\beta-\gamma}\left[\int_{0}^{\infty}(1+\mu)^{-1} \mu^{1-\beta-\gamma} \mathrm{d} \mu\right]\|x\|_{(X, \mathscr{D}(A))_{\gamma, p}} .
$$

Then $w \in X_{A}^{\gamma+\beta-1, \infty}$, for the integral on the right-hand side of (5.12) is convergent for $\gamma \in(1-\beta, 1)$. In conclusion, since (4.25) with $\left(p_{1}, p_{2}\right)=(p, \infty)$ implies $x \in(X, \mathscr{D}(A))_{\gamma, p} \hookrightarrow X_{A}^{\gamma+\beta-1, \infty}$, we have $w-x \in\left[X_{A}^{\gamma+\beta-1, \infty} \cap A 0\right]=\{0\}$. Recalling (5.9), this completes the proof of the strong continuity of $\mathrm{e}^{t A}$ in the norm of $X$ on 
$(X, \mathscr{D}(A))_{\gamma, p}, \gamma \in(1-\beta, 1), p \in[1, \infty]$. Finally, due to (4.8), the strong continuity of $\mathrm{e}^{t A}$ in the norm of $X$ on $X_{A}^{\gamma, p}, \gamma \in(1-\beta, 1), p \in[1, \infty]$, follows from that on $(X, \mathscr{D}(A))_{\gamma, p}$. The proof is complete.

We are now ready to improve (5.2).

Corollary 5.3. Let $A$ be a $m$. l. operator satisfying $(\mathrm{H} 1)$ with $\alpha+\beta>1$ and let $Y_{\gamma}^{p}$ be as in Lemma 5.1. Then, there exists a positive constant $c_{15}=$ $c_{15}(\alpha, \beta, \gamma, p)$ such that:

$$
\left\|\mathrm{e}^{t A}-I\right\|_{\mathscr{L}\left(Y_{\gamma}^{p} ; X\right)} \leq c_{15} t^{(\alpha+\beta+\gamma-2) / \alpha}, \quad t>0, \gamma \in(2-\alpha-\beta, 1), p \in[1, \infty] .
$$

Proof. Let $x \in Y_{\gamma}^{p}, \gamma \in(2-\alpha-\beta, 1), p \in[1, \infty]$, where $Y_{\gamma}^{p} \in\left\{(X, \mathscr{D}(A))_{\gamma, p}\right.$, $\left.X_{A}^{\gamma, p}\right\}$. Since $(2-\alpha-\beta, 1) \subseteq(1-\beta, 1)$, Proposition 5.2 allows us to write

$$
\mathrm{e}^{t A} x-x=\int_{0}^{t}\left[D_{t} \mathrm{e}^{t A}\right]_{t=\tau} x \mathrm{~d} \tau=-\int_{0}^{t}\left[(-A)^{1}\right]^{\circ} \mathrm{e}^{\tau A} x \mathrm{~d} \tau, \quad t>0 .
$$

From (5.3) it thus follows

$$
\left\|\mathrm{e}^{t A} x-x\right\|_{X} \leq c_{12}\left[\int_{0}^{t} \tau^{(\beta+\gamma-2) / \alpha} \mathrm{d} \tau\right]\|x\|_{Y_{\gamma}^{p}} \leq c_{15} t^{(\alpha+\beta+\gamma-2) / \alpha}\|x\|_{Y_{\gamma}^{p}},
$$

where $c_{15}=\alpha(\alpha+\beta+\gamma-2)^{-1} c_{12}$. This completes the proof.

Corollary 5.4. Let $A$ and $Y_{\gamma}^{p}$ be as in Corollary 5.3. If $\gamma \in(2-\alpha-\beta, 1)$, then

$$
\left\|\mathrm{e}^{t A}-\mathrm{e}^{s A}\right\|_{\mathscr{L}\left(Y_{\gamma}^{p} ; X\right)} \leq c_{15}(t-s)^{(\alpha+\beta+\gamma-2) / \alpha}, \quad t>s \geq 0, p \in[1, \infty]
$$

Proof. The case $s=0$ following from Corollary 5.3 and the definition $\mathrm{e}^{0 A}=I$, it suffices to consider the case $t>s>0$. To this purpose, we replace (5.14) with $\mathrm{e}^{t A} x-\mathrm{e}^{s A} x=-\int_{s}^{t}\left[(-A)^{1}\right]^{\circ} \mathrm{e}^{\tau A} x \mathrm{~d} \tau, t>s>0$. Then, taking into account the inequality $t^{v}-s^{v} \leq(t-s)^{v}, v \in[0,1]$, the assertion follows reasoning as in the derivation of (5.15).

REMARK 5.5. To show that estimate (5.3), and, consequently, (5.13) and (5.16), does not depend on the interpolation method used in the proof of Lemma 5.1 , here we provide a different proof of (5.3), in which we make use only of definition (3.19) and estimate (4.18). First, using $z(z I-A)^{-1}=A^{\circ}(z I-A)^{-1}+I$, 
$z \in \rho(A)$, and $\int_{\Gamma} \mathrm{e}^{t \lambda} \mathrm{d} \lambda=0$, we rewrite $\left[(-A)^{1}\right]^{\circ} \mathrm{e}^{t A} x, \quad t>0, \quad x \in(X, \mathscr{D}(A))_{\gamma, p}$, $\gamma \in(0,1), p \in[1, \infty]$, as follows

$$
\left[(-A)^{1}\right]^{\circ} \mathrm{e}^{t A} x=-\frac{1}{2 \pi i} \int_{\Gamma} \mathrm{e}^{t \lambda} A^{\circ}(\lambda I-A)^{-1} x \mathrm{~d} \lambda .
$$

Due to (4.18), from (5.17) we thus obtain

$$
\left\|\left[(-A)^{1}\right]^{\circ} \mathrm{e}^{t A} x\right\|_{X} \leq(2 \pi)^{-1} c_{3}(\gamma, p)\left[\int_{\Gamma} \mathrm{e}^{t \Re \mathrm{e} \lambda}(|\lambda|+1)^{1-\beta-\gamma}|\mathrm{d} \lambda|\right]\|x\|_{(X, \mathscr{D}(A))_{\gamma, p}} .
$$

Now, recalling that $|\lambda| \geq c>0$ for every $\lambda \in \Gamma$, we have $|\lambda| \leq|\lambda|+1 \leq$ $\left(1+c^{-1}\right)|\lambda|, \lambda \in \Gamma$. As a consequence, the following inequality holds

$$
(|\lambda|+1)^{1-\beta-\gamma} \leq c_{\beta, \gamma}|\lambda|^{1-\beta-\gamma}, \quad \forall \lambda \in \Gamma,
$$

where $c_{\beta, \gamma}=\left(1+c^{-1}\right)^{1-\beta-\gamma}$ or $c_{\beta, \gamma}=1$ according that $\gamma \in(0,1-\beta)$ or $\gamma \in$ $[1-\beta, 1)$. Moreover, $\Re \mathrm{e} \lambda=-c(|\Im \mathrm{m} \lambda|+1)^{\alpha} \geq-c\left(1+c^{-1}\right)^{\alpha}|\lambda|^{\alpha}, \lambda \in \Gamma$. Then, replacing (5.19) in (5.18) and setting $c_{16}=\pi^{-1} c_{\beta, \gamma} c_{3}(\gamma, p)$, we get

$$
\left\|\left[(-A)^{1}\right]^{\circ} \mathrm{e}^{t A} x\right\|_{X} \leq c_{16}\left[\int_{0}^{\infty} \mathrm{e}^{-c_{\alpha} t \eta^{\alpha}} \eta^{1-\beta-\gamma} \mathrm{d} \eta\right]\|x\|_{(X, \mathscr{D}(A))_{\gamma, p}},
$$

where $c_{\alpha}=c\left(1+c^{-1}\right)^{\alpha}$. Finally, the transformation $c_{\alpha} t \eta^{\alpha}=s$ in the latter integral yields

$$
\left\|\left[(-A)^{1}\right]^{\circ} \mathrm{e}^{t A} x\right\|_{X} \leq c_{16} \alpha^{-1} E((2-\beta-\gamma) / \alpha)\left(c_{\alpha} t\right)^{(\beta+\gamma-2) / \alpha}\|x\|_{(X, \mathscr{D}(A))_{\gamma, p}},
$$

$E(\chi), \chi>0$, being the Euler's gamma function.

\section{Intermediate Properties of the Domains $\mathscr{D}\left((-A)^{\theta}\right)$}

Let $A$ be a m. 1. operator satisfying assumption (H1). On the basis of (5.3), for every $\gamma \in(0,1)$ and $p \in[1, \infty]$ we now introduce the Banach spaces $\mathscr{D}_{A}(\gamma, p)$ defined by:

$$
\left\{\begin{array}{l}
\mathscr{D}_{A}(\gamma, p)=\left\{x \in X:[x]_{\mathscr{D}_{A}(\gamma, p)}:=\left\|\xi^{(2-\beta-\gamma) / \alpha}\left[(-A)^{1}\right]^{\circ} \mathrm{e}^{\xi A} x\right\|_{L_{p}^{*}(X)}<\infty\right\}, \\
\|x\|_{\mathscr{D}_{A}(\gamma, p)}=\|x\|_{X}+[x]_{\mathscr{D}_{A}(\gamma, p)} .
\end{array}\right.
$$

As we have noticed in Section 2, if $A$ is single-valued and $\beta=1$ in (H1), then the semigroup $\mathrm{e}^{t A}$ is analytic in some sector containing the positive real axis. In this case it is well-known that the spaces $\mathscr{D}_{A}(\gamma, p)$ coincide, in the sense of equivalence of the norms, with the real interpolation spaces $(X, \mathscr{D}(A))_{\gamma, p}$ (cf. [5, Theorem 3, 
with $\alpha$ being replaced by $1-\gamma-1 / p]$, [27, Proposition 2.2.2] and [35, Theorem 1.14.5]). If $A$ is really a m. 1 . operator, i.e. $\{0\} \subsetneq A 0$, the things become considerably worse. Indeed, contrarily to the identities $\left[X_{A}^{\gamma, p} \cap A 0\right]=\{0\}, \gamma \in(0,1)$, and $\left[(X, \mathscr{D}(A))_{\gamma, p} \cap A 0\right]=\{0\}, \gamma \in(1-\beta, 1)$, which we have verified in Remarks 4.2 and 4.6, the spaces $\mathscr{D}_{A}(\gamma, p)$ contain $A 0$. For (cf. (3.20)), if $x \in A 0 \subseteq$ $\bigcap_{t>0} \mathscr{N}\left(\left[(-A)^{1}\right]^{\circ} \mathrm{e}^{t A}\right)$, then $[x]_{\mathscr{D}_{A}(\gamma, p)}=0$.

Our proof of the multivalued version of (4.26) will deeply depends on the forthcoming Proposition 6.3, which estabilishes some inclusion relations between the spaces $(X, \mathscr{D}(A))_{\gamma, p}, X_{A}^{\gamma, p}$ and $\mathscr{D}_{A}(\gamma, p)$ and exhibits the special role played by the linear subspace $A 0$. We begin by proving the following Lemmas 6.1 and 6.2.

Lemma 6.1. For every $\mu \in \rho(A)$ and every $x \in X$ it holds that:

$$
\left\{\mu \mathrm{e}^{t A}+\left[(-A)^{1}\right]^{\circ} \mathrm{e}^{t A}\right\} A^{\circ}(\mu I-A)^{-1} x=-\left[(-A)^{1}\right]^{\circ} \mathrm{e}^{t A} x, \quad t>0 .
$$

Proof. Using (3.19) with $\theta=0$ and $\theta=1$, we rewrite the left-hand side of (6.1) as follows

$$
\begin{aligned}
\left\{\mu \mathrm{e}^{t A}\right. & \left.+\left[(-A)^{1}\right]^{\circ} \mathrm{e}^{t A}\right\} A^{\circ}(\mu I-A)^{-1} x \\
& =\left\{\frac{1}{2 \pi i} \int_{\Gamma} \mu \mathrm{e}^{t \lambda}(\lambda I-A)^{-1} \mathrm{~d} \lambda+\frac{1}{2 \pi i} \int_{\Gamma}(-\lambda) \mathrm{e}^{t \lambda}(\lambda I-A)^{-1} \mathrm{~d} \lambda\right\} A^{\circ}(\mu I-A)^{-1} x \\
& =\frac{1}{2 \pi i} \int_{\Gamma}(\mu-\lambda) \mathrm{e}^{t \lambda}(\lambda I-A)^{-1} A^{\circ}(\mu I-A)^{-1} x \mathrm{~d} \lambda, \quad t>0, x \in X .
\end{aligned}
$$

Then, using (2.7) and $\int_{\Gamma} \mathrm{e}^{t \lambda} \mathrm{d} \lambda=0, t>0$, and recalling (5.17), we obtain

$$
\begin{aligned}
\left\{\mu \mathrm{e}^{t A}\right. & \left.+\left[(-A)^{1}\right]^{\circ} \mathrm{e}^{t A}\right\} A^{\circ}(\mu I-A)^{-1} x \\
& =\frac{1}{2 \pi i} \int_{\Gamma} \mathrm{e}^{t \lambda}\left[A^{\circ}(\lambda I-A)^{-1} x-A^{\circ}(\mu I-A)^{-1} x\right] \mathrm{d} \lambda \\
& =\frac{1}{2 \pi i} \int_{\Gamma} \mathrm{e}^{t \lambda} A^{\circ}(\lambda I-A)^{-1} x \mathrm{~d} \lambda=-\left[(-A)^{1}\right]^{\circ} \mathrm{e}^{t A} x, \quad t>0, x \in X .
\end{aligned}
$$

This completes the proof of (6.1).

Lemma 6.2. Let $\alpha$ and $\beta$ in assumption $(\mathrm{H} 1)$ be such that $\alpha+\beta>1$ and let $x \in\{0\} \cup\left[D_{A}(\gamma, p) \backslash A 0\right], \gamma \in(2-\alpha-\beta, 1), p \in[1, \infty]$. Then

$$
\int_{0}^{\infty} \mathrm{e}^{-z t}\left[(-A)^{1}\right]^{\circ} \mathrm{e}^{t A} x \mathrm{~d} t=-A^{\circ}(z I-A)^{-1} x, \quad \forall \Re \mathrm{e} z>0 .
$$


Proof. First, observe that, due to (3.20), if $x \in A 0$, then the left-hand side of (6.2) is the zero element of $X$ whereas the right-hand side reduces to $-A^{\circ}(z I-A)^{-1} x=-z(z I-A)^{-1} x+x=x$. Therefore, if $x \in A 0$, then (6.2) fails, unless $x=0$. This agrees with the identity $\mathscr{N}\left(A^{\circ}(z I-A)^{-1}\right)=\{0\}$ established in Lemma 2.1 and explains why, in general, the linear subspace $A 0 \backslash\{0\}$ should be removed from $D_{A}(\gamma, p)$. Let us now show that the integral in (6.2) converges for $x \in D_{A}(\gamma, p), \gamma \in(2-\alpha-\beta, 1), p \in[1, \infty]$. We have already noticed that, if $x \in A 0$, then our integral is the zero element of $X$. If $x \in D_{A}(\gamma, p) \backslash A 0$, then, denoting with $p^{\prime}$ the conjugate exponent of $p$, from Hölder's inequality and the definition of seminorm $[\cdot]_{\mathscr{D}_{A}(\gamma, p)}$ we get

$$
\begin{aligned}
& \left\|\int_{0}^{\infty} \mathrm{e}^{-z t}\left[(-A)^{1}\right]^{\circ} \mathrm{e}^{t A} x \mathrm{~d} t\right\|_{X} \\
& \quad \leq \int_{0}^{\infty} \mathrm{e}^{-t \Re \mathrm{e} z}\left\|\left[(-A)^{1}\right]^{\circ} \mathrm{e}^{t A} x\right\|_{X} \mathrm{~d} t \\
& \quad \leq \int_{0}^{\infty} \mathrm{e}^{-t \Re \mathrm{e} z} t^{[(\beta+\gamma-2) / \alpha]+1 / p} t^{[(2-\beta-\gamma) / \alpha]-1 / p}\left\|\left[(-A)^{1}\right]^{\circ} \mathrm{e}^{t A} x\right\|_{X} \mathrm{~d} t \\
& \quad \leq\left(\int_{0}^{\infty} \mathrm{e}^{-p^{\prime} t \Re \mathrm{e} z} t^{\left[(\beta+\gamma-2) p^{\prime} / \alpha\right]+p^{\prime} / p} \mathrm{~d} t\right)^{1 / p^{\prime}}[x]_{\mathscr{D}_{A}(\gamma, p)},
\end{aligned}
$$

Since $p^{\prime} / p=p^{\prime}-1$, the transformation $p^{\prime} t \Re \mathrm{e} z=\tau$ leads to

$$
\begin{aligned}
& \int_{0}^{\infty} \mathrm{e}^{-p^{\prime} t \Re \mathrm{e} z} t^{\left[(\beta+\gamma-2) p^{\prime} / \alpha\right]+p^{\prime} / p} \mathrm{~d} t \\
& \quad=\left(p^{\prime} \Re \mathrm{e} z\right)^{(2-\alpha-\beta-\gamma) p^{\prime} / \alpha} E\left((\alpha+\beta+\gamma-2) p^{\prime} / \alpha\right),
\end{aligned}
$$

where $E(\chi), \chi>0$, is the Euler's gamma function. As a consequence, from (6.3) we obtain that for every $\Re \mathrm{e} z>0$ and every $x \in \mathscr{D}_{A}(\gamma, p) \backslash A 0, \gamma \in(2-\alpha-\beta, 1)$, $p \in[1, \infty]$ :

$$
\left\|\int_{0}^{\infty} \mathrm{e}^{-z t}\left[(-A)^{1}\right]^{\circ} \mathrm{e}^{t A} x \mathrm{~d} t\right\|_{X} \leq\left[c_{17}\left(\alpha, \beta, \gamma, p^{\prime}, \Re \mathrm{e} z\right)\right]^{1 / p^{\prime}}[x]_{\mathscr{D}_{A}(\gamma, p)}<\infty .
$$

where $c_{17}\left(\alpha, \beta, \gamma, p^{\prime}, \Re \mathrm{e} z\right)$ is the constant on the right-hand side of (6.4). Let us now prove (6.2). Clearly, if $x=0$, then there is nothing to prove. If $x \in\left[\mathscr{D}_{A}(\gamma, p) \backslash A 0\right], \gamma \in(2-\alpha-\beta, 1), \quad p \in[1, \infty]$, then, substituting identity (6.1) with $\mu$ being replaced by $z$, the left-hand side of (6.2) can be rewritten as 


$$
\begin{aligned}
\int_{0}^{\infty} \mathrm{e}^{-z t}\left[(-A)^{1}\right]^{\circ} \mathrm{e}^{t A} x \mathrm{~d} t= & \int_{0}^{\infty} \mathrm{e}^{-z t}\left\{-z \mathrm{e}^{t A}-\left[(-A)^{1}\right]^{\circ} \mathrm{e}^{t A}\right\} A^{\circ}(z I-A)^{-1} x \mathrm{~d} t \\
= & -\int_{0}^{\infty} z \mathrm{e}^{-z t} \mathrm{e}^{t A} A^{\circ}(z I-A)^{-1} x \mathrm{~d} t \\
& -\int_{0}^{\infty} \mathrm{e}^{-z t}\left[(-A)^{1}\right]^{\circ} \mathrm{e}^{t A} A^{\circ}(z I-A)^{-1} x \mathrm{~d} t .
\end{aligned}
$$

Notice that, if $x \in A 0$, then $A^{\circ}(z I-A)^{-1} x=-x \in A 0$ and the first integral on the right-hand side of (6.6) becomes zero in $X$. In fact, from Lemma 3.9 we have $A 0=\bigcap_{t>0} \mathscr{N}\left(\mathrm{e}^{t A}\right)$. Thus, when $x \in A 0,(6.6)$ reduces to $\int_{0}^{\infty} \mathrm{e}^{-z t}\left[(-A)^{1}\right]^{\circ} \mathrm{e}^{t A} x \mathrm{~d} t=$ $-\int_{0}^{\infty} \mathrm{e}^{-z t}\left[(-A)^{1}\right]^{\circ} \mathrm{e}^{t A}(-x) \mathrm{d} t=0$. On the contrary, if $x \notin A 0$, then Lemma 2.1 implies $A^{\circ}(z I-A)^{-1} x \notin A 0=\mathscr{N}\left((z I-A)^{-1}\right)$ and from identity (3.24) it follows that

$$
\int_{0}^{\infty} z \mathrm{e}^{-z t} \mathrm{e}^{t A} A^{\circ}(z I-A)^{-1} x \mathrm{~d} t=z(z I-A)^{-1} A^{\circ}(z I-A)^{-1} x \neq 0 .
$$

Hence, when $x \notin A 0$, the first integral on the right-hand side of (6.6) is different from the zero element of $X$ and an integration by parts yields

$$
\begin{aligned}
& -\int_{0}^{\infty} z \mathrm{e}^{-z t} \mathrm{e}^{t A} A^{\circ}(z I-A)^{-1} x \mathrm{~d} t \\
& \quad=\left[\mathrm{e}^{-z t} \mathrm{e}^{t A}\right]_{t=0}^{t=\infty} A^{\circ}(z I-A)^{-1} x-\int_{0}^{\infty} \mathrm{e}^{-z t} D_{t} \mathrm{e}^{t A} A^{\circ}(z I-A)^{-1} x \mathrm{~d} t \\
& =-A^{\circ}(z I-A)^{-1} x+\int_{0}^{\infty} \mathrm{e}^{-z t}\left[(-A)^{1}\right]^{\circ} \mathrm{e}^{t A} A^{\circ}(z I-A)^{-1} x \mathrm{~d} t
\end{aligned}
$$

where we have used $\lim _{t \rightarrow \infty} \mathrm{e}^{-z t} \mathrm{e}^{t A}=0, \Re \mathrm{e} z>0$, which follows from estimate (3.23) with $\theta=0$. Replacing (6.7) in (6.6) we finally obtain (6.2).

Proposition 6.3. Let $A$ be a m. l. operator satisfying (H1). Then, for every $\gamma \in(0,1)$ :

$$
\left\{\begin{array}{l}
X_{A}^{\gamma, p} \hookrightarrow(X, \mathscr{D}(A))_{\gamma, p} \hookrightarrow \mathscr{D}_{A}(\alpha \gamma, p), \quad p \in[1, \infty) \\
X_{A}^{\gamma, \infty} \hookrightarrow(X, \mathscr{D}(A))_{\gamma, \infty} \hookrightarrow \mathscr{D}_{A}(\gamma, \infty) .
\end{array}\right.
$$

Further, if $\alpha+\beta>1$, then for every $\gamma \in(2-\alpha-\beta, 1)$ and every $\delta \in$ $(0,(\alpha+\beta+\gamma-2) / \alpha)^{1}$ :

\footnotetext{
${ }^{1}$ Here $(\alpha+\beta+\gamma-2) / \alpha<1$, since $\gamma<1 \leq 2-\beta$.
} 


$$
\left\{\begin{array}{l}
\{0\} \cup\left[\mathscr{D}_{A}(\gamma, p) \backslash A 0\right] \hookrightarrow X_{A}^{\delta, p} \hookrightarrow(X, \mathscr{D}(A))_{\delta, p}, \quad p \in[1, \infty), \\
\{0\} \cup\left[\mathscr{D}_{A}(\gamma, \infty) \backslash A 0\right] \hookrightarrow X_{A}^{(\alpha+\beta+\gamma-2) / \alpha, \infty} \hookrightarrow(X, \mathscr{D}(A))_{(\alpha+\beta+\gamma-2) / \alpha, \infty} .
\end{array}\right.
$$

Here $\{0\} \cup\left[\mathscr{D}_{A}(\gamma, p) \backslash A 0\right]$ is a normed linear space over $\mathbf{C}$ endowed with the norm $\|\cdot\|_{\mathscr{D}_{A}(\gamma, p)}$.

Proof. Due to (4.8), it suffices to prove the embeddings on the right of (6.8) and on the left of (6.9). First, let $x \in(X, \mathscr{D}(A))_{\gamma, \infty}, \gamma \in(0,1)$. Then from (5.3) we have

$$
[x]_{\mathscr{D}_{A}(\gamma, \infty)}=\sup _{t>0}\left\|t^{(2-\beta-\gamma) / \alpha}\left[(-A)^{1}\right]^{\circ} \mathrm{e}^{t A} x\right\|_{X} \leq c_{12}\|x\|_{(X, \mathscr{D}(A))_{\gamma, \infty}},
$$

proving the second in (6.8). Now, let $x \in(X, \mathscr{D}(A))_{\gamma, p}, \gamma \in(0,1), p \in[1, \infty)$. We write

$$
[x]_{\mathscr{D}_{A}(\alpha \gamma, p)}^{p}=I_{1}+I_{2},
$$

where

$$
\begin{aligned}
& I_{1}=\int_{0}^{1} \tau^{(2-\beta-\alpha \gamma) p / \alpha}\left\|\left[(-A)^{1}\right]^{\circ} \mathrm{e}^{\tau A} x\right\|_{X}^{p} \frac{\mathrm{d} \tau}{\tau}, \\
& I_{2}=\int_{1}^{\infty} \tau^{(2-\beta-\alpha \gamma) p / \alpha}\left\|\left[(-A)^{1}\right]^{\circ} \mathrm{e}^{\tau A} x\right\|_{X}^{p} \frac{\mathrm{d} \tau}{\tau} .
\end{aligned}
$$

Consider first $I_{1}$. To this purpose we recall that the transformation $\xi=\tau^{-1}$ in definition (4.1) leads to the following equivalent characterization of the spaces $(X, \mathscr{D}(A))_{\gamma, p}$ :

$$
\left\{\begin{aligned}
(X, \mathscr{D}(A))_{\gamma, p}= & \left\{x \in X: x=u_{0}(\tau)+u_{1}(\tau), \tau \in(0, \infty),\right. \\
& u_{0} \in C((0, \infty) ; X), u_{1} \in C((0, \infty) ; \mathscr{D}(A)), \\
& \left.\left\|\tau^{-\gamma} u_{0}\right\|_{L_{p}^{*}(X)}+\left\|\tau^{1-\gamma} u_{1}\right\|_{L_{p}^{*}(\mathscr{D}(A))}<\infty\right\}, \\
\|x\|_{(X, \mathscr{D}(A))_{\gamma, p}}= & \inf _{u_{0}, u_{1}}\left\{\left\|\tau^{-\gamma} u_{0}\right\|_{L_{p}^{*}(X)}+\left\|\tau^{1-\gamma} u_{1}\right\|_{L_{p}^{*}(\mathscr{D}(A))}\right\} .
\end{aligned}\right.
$$

So, with any pair of functions $u_{0}$ and $u_{1}$ satisfying the condition in (6.13), from (6.11) and inequality $(a+b)^{p} \leq 2^{p-1}\left(a^{p}+b^{p}\right), a, b \geq 0, p \in[1, \infty)$, we get:

$$
I_{1} \leq 2^{p-1}\left[I_{1,0}+I_{1,1}\right]
$$

where

$$
I_{1, j}=\int_{0}^{1} \tau^{(2-\beta-\alpha \gamma) p / \alpha}\left\|\left[(-A)^{1}\right]^{\circ} \mathrm{e}^{\tau A} u_{j}(\tau)\right\|_{X}^{p} \frac{\mathrm{d} \tau}{\tau}, \quad j=0,1 .
$$


Applying (5.5) to $I_{1,0}$ we obtain

$$
I_{1,0} \leq\left(\tilde{c}_{\alpha, \beta, 1}\right)^{p} \int_{0}^{1} \tau^{-\gamma p}\left\|u_{0}(\tau)\right\|_{X}^{p} \frac{\mathrm{d} \tau}{\tau} \leq\left(\tilde{c}_{\alpha, \beta, 1}\right)^{p}\left\|\tau^{-\gamma} u_{0}\right\|_{L_{p}^{*}(X)}^{p} .
$$

Instead, (5.4) applied to $I_{1,1}$ yields:

$$
I_{1,1} \leq\left(\tilde{c}_{\alpha, \beta, 0}\right)^{p} \int_{0}^{1} \tau^{(1-\alpha \gamma) p / \alpha}\left\|u_{1}(\tau)\right\|_{\mathscr{D}(A)}^{p} \frac{\mathrm{d} \tau}{\tau} .
$$

Since $(1-\alpha \gamma) / \alpha=(1-\gamma)+(1-\alpha) / \alpha$ and $1-\alpha \geq 0$, from (6.16) we thus find

$$
\begin{aligned}
I_{1,1} & \leq\left(\tilde{c}_{\alpha, \beta, 0}\right)^{p} \int_{0}^{1} \tau^{(1-\gamma) p} \tau^{(1-\alpha) p / \alpha}\left\|u_{1}(\tau)\right\|_{\mathscr{D}(A)}^{p} \frac{\mathrm{d} \tau}{\tau} \\
& \leq\left(\tilde{c}_{\alpha, \beta, 0}\right)^{p} \int_{0}^{1} \tau^{(1-\gamma) p}\left\|u_{1}(\tau)\right\|_{\mathscr{D}(A)}^{p} \frac{\mathrm{d} \tau}{\tau} \leq\left(\tilde{c}_{\alpha, \beta, 0}\right)^{p}\left\|\tau^{1-\gamma} u_{1}\right\|_{L_{p}^{*}(\mathscr{D}(A))}^{p}
\end{aligned}
$$

Therefore, summing up (6.14), (6.15) and (6.17) and taking the infimum over all possible representations of the form $x=u_{0}(\tau)+u_{1}(\tau)$, we have

$$
I_{1} \leq\left[c_{18}(\alpha, \beta, p)\right]^{p}\|x\|_{(X, \mathscr{D}(A))_{\gamma, p}}^{p},
$$

where $c_{18}(\alpha, \beta, p)=2^{(p-1) / p} \max _{k=0,1} \tilde{c}_{\alpha, \beta, k}$. Consider now $I_{2}$ defined by (6.12). From (5.5) and $\|x\|_{X} \leq c_{2}(\gamma, p)\|x\|_{(X, \mathscr{D}(A))_{\gamma, p}}$ it follows that

$$
I_{2} \leq\left(\tilde{c}_{\alpha, \beta, 1}\right)^{p}\left[\int_{1}^{\infty} \tau^{-\gamma p-1} \mathrm{~d} \tau\right]\|x\|_{X}^{p} \leq\left[c_{19}(\alpha, \beta, \gamma, p)\right]^{p}\|x\|_{(X, \mathscr{D}(A))_{\gamma, p}}^{p},
$$

where $c_{19}(\alpha, \beta, \gamma, p)=\tilde{c}_{\alpha, \beta, 1}(\gamma p)^{-1 / p} c_{2}(\gamma, p)$. From (6.10), (6.18) and (6.19) we derive

$$
[x]_{\mathscr{D}_{A}(\alpha \gamma, p)}^{p} \leq\left[c_{20}(\alpha, \beta, \gamma, p)\right]^{p}\|x\|_{(X, \mathscr{D}(A))_{\gamma, p}}^{p},
$$

where $c_{20}(\alpha, \beta, \gamma, p)=\left\{\left[c_{18}(\alpha, \beta, p)\right]^{p}+\left[c_{19}(\alpha, \beta, \gamma, p)\right]^{p}\right\}^{1 / p}$. Consequently

$$
\|x\|_{\mathscr{D}_{A}(\alpha \gamma, p)} \leq c_{21}(\alpha, \beta, \gamma, p)\|x\|_{(X, \mathscr{D}(A))_{\gamma, p}}, \quad \gamma \in(0,1), p \in[1, \infty),
$$

where $c_{21}(\alpha, \beta, \gamma, p)=c_{2}(\gamma, p)+c_{20}(\alpha, \beta, \gamma, p)$. This completes the proof of (6.8). We now assume $\alpha+\beta>1$ and prove (6.9). Let first $x \in\{0\} \cup\left[\mathscr{D}_{A}(\gamma, \infty) \backslash A 0\right]$, $\gamma \in(2-\alpha-\beta, 1)$. Then, from (6.2) and (6.5) with $\left(z, p, p^{\prime}\right)=(\xi, \infty, 1)$ it follows that for every $\xi>0$ :

$$
\left\|A^{\circ}(\xi I-A)^{-1} x\right\|_{X} \leq \xi^{(2-\alpha-\beta-\gamma) / \alpha} E((\alpha+\beta+\gamma-2) / \alpha)[x]_{\mathscr{D}_{A}(\gamma, \infty)},
$$


where $E(\chi), \chi>0$, is the Euler's gamma function. Therefore

$$
\begin{aligned}
\|x\|_{X_{A}^{(\alpha+\beta+\gamma-2) / \alpha, \infty}} & =\|x\|_{X}+\sup _{\xi>0} \xi^{(\alpha+\beta+\gamma-2) / \alpha}\left\|A^{\circ}(\xi I-A)^{-1} x\right\|_{X} \\
& \leq \max \{1, E((\alpha+\beta+\gamma-2) / \alpha)\}\|x\|_{\mathscr{D}_{A}(\gamma, \infty)},
\end{aligned}
$$

proving that $x \in X_{A}^{(\alpha+\beta+\gamma-2) / \alpha, \infty}$. Let us show that $\{0\} \cup\left[\mathscr{D}_{A}(\gamma, \infty) \backslash A 0\right], \quad \gamma \in$ $(2-\alpha-\beta, 1)$, is a normed linear space over $\mathbf{C}$ endowed with the norm $\|\cdot\|_{\mathscr{D}_{A}(\gamma, \infty)}$. First, let $\lambda \in \mathbf{C}$ and $x \in\{0\} \cup\left[\mathscr{D}_{A}(\gamma, \infty) \backslash A 0\right], \gamma \in(2-\alpha-\beta, 1)$. Then $\lambda x \in\{0\} \cup\left[\mathscr{D}_{A}(\gamma, \infty) \backslash A 0\right]$. For, if $\lambda x=y \in A 0 \backslash\{0\}, \lambda \in \mathbf{C} \backslash\{0\}$, then $x=\lambda^{-1} y \in$ $A 0 \backslash\{0\}$, which is a contradiction. Now, let $x_{1}, x_{2} \in\{0\} \cup\left[\mathscr{D}_{A}(\gamma, \infty) \backslash A 0\right], \gamma \in$ $(2-\alpha-\beta, 1)$. Then, since $x_{1}, x_{2} \in X_{A}^{(\alpha+\beta+\gamma-2) / \alpha, \infty}$ due to (6.20), we have $x_{1}+x_{2} \in X_{A}^{(\alpha+\beta+\gamma-2) / \alpha, \infty}$. From $\left[X_{A}^{\sigma, p} \cap A 0\right]=\{0\}, \sigma \in(0,1), p \in[1, \infty]$, it thus follows that $x_{1}+x_{2} \in A 0$ only if $x_{1}+x_{2}=0$, i.e. $x_{1}=-x_{2}$. Since $x_{1}+x_{2}$ obviously belongs to $\mathscr{D}_{A}(\gamma, \infty)$, we conclude that $x_{1}+x_{2} \in\{0\} \cup\left[\mathscr{D}_{A}(\gamma, \infty) \backslash A 0\right]$. This completes the proof of the second of (6.9). Now, let $x \in\{0\} \cup\left[\mathscr{D}_{A}(\gamma, p) \backslash A 0\right]$, $\gamma \in(2-\alpha-\beta, 1), p \in[1, \infty)$. For every $\delta \in(0,(\alpha+\beta+\gamma-2) / \alpha)$ we write

$$
[x]_{X_{A}^{\delta, p}}^{p}=I_{3}+I_{4}
$$

where

$$
I_{3}=\int_{0}^{1} \xi^{\delta p}\left\|A^{\circ}(\xi I-A)^{-1} x\right\|_{X}^{p} \frac{\mathrm{d} \xi}{\xi}, \quad I_{4}=\int_{1}^{\infty} \xi^{\delta p}\left\|A^{\circ}(\xi I-A)^{-1} x\right\|_{X}^{p} \frac{\mathrm{d} \xi}{\xi} .
$$

Using (4.17) we obtain

$$
\begin{aligned}
I_{3} & \leq(C+1)^{p}\left[\int_{0}^{1} \xi^{\delta p-1}(\xi+1)^{(1-\beta) p} \mathrm{~d} \xi\right]\|x\|_{X}^{p} \\
& \leq 2^{(1-\beta) p}(C+1)^{p}\left[\int_{0}^{1} \xi^{\delta p-1} \mathrm{~d} \xi\right]\|x\|_{X}^{p}=\left[c_{21}(\beta, \delta, p)\right]^{p}\|x\|_{X}^{p} .
\end{aligned}
$$

where $c_{21}(\beta, \delta, p)=2^{(1-\beta)}(C+1)(\delta p)^{-1 / p}$. As far as $I_{4}$ is concerned, first, using (6.2) and (6.5) we derive for every $\xi>0$ :

$$
\left\|A^{\circ}(\xi I-A)^{-1} x\right\|_{X} \leq c_{22}\left(\alpha, \beta, \gamma, p^{\prime}\right) \xi^{(2-\alpha-\beta-\gamma) / \alpha}[x]_{\mathscr{D}_{A}(\gamma, p)},
$$

where $c_{22}\left(\alpha, \beta, \gamma, p^{\prime}\right)=\left(p^{\prime}\right)^{(2-\alpha-\beta-\gamma) / \alpha}\left[E\left((\alpha+\beta+\gamma-2) p^{\prime} / \alpha\right)\right]^{1 / p^{\prime}}$. Then

$$
\begin{aligned}
I_{4} & \leq\left[c_{22}\left(\alpha, \beta, \gamma, p^{\prime}\right)\right]^{p}\left[\int_{1}^{\infty} \xi^{[\delta+(2-\alpha-\beta-\gamma) / \alpha] p-1} \mathrm{~d} \xi\right][x]_{\mathscr{D}_{A}(\gamma, p)}^{p} \\
& \leq\left[c_{23}\left(\alpha, \beta, \gamma, \delta, p, p^{\prime}\right)\right]^{p}[x]_{\mathscr{D}_{A}(\gamma, p)}^{p},
\end{aligned}
$$


where $c_{23}\left(\alpha, \beta, \gamma, \delta, p, p^{\prime}\right)=c_{22}\left(\alpha, \beta, \gamma, p^{\prime}\right) \alpha^{1 / p}[(\alpha+\beta+\gamma-2-\alpha \delta) p]^{-1 / p}$. Therefore, summing up (6.21)-(6.23) we get

$$
[x]_{X_{A}^{\delta, p}}^{p} \leq\left[c_{24}\left(\alpha, \beta, \gamma, \delta, p, p^{\prime}\right)\right]^{p}\|x\|_{\mathscr{D}_{A}(\gamma, p)}^{p}
$$

where $c_{24}\left(\alpha, \beta, \gamma, \delta, p, p^{\prime}\right)=\max \left\{c_{21}(\beta, \delta, p), c_{23}\left(\alpha, \beta, \gamma, \delta, p, p^{\prime}\right)\right\}$. Consequently

$$
\|x\|_{X_{A}^{\delta, p}}=\|x\|_{X}+[x]_{X_{A}^{\delta, p}} \leq\left[1+c_{24}\left(\alpha, \beta, \gamma, \delta, p, p^{\prime}\right)\right]\|x\|_{\mathscr{D}_{A}(\gamma, p)},
$$

proving that $x \in X_{A}^{\delta, p}, \delta \in(0,(\alpha+\beta+\gamma-2) / \alpha)$. The same reasonings made before for $p=\infty$ now show that $\{0\} \cup\left[\mathscr{D}_{A}(\gamma, p) \backslash A 0\right], \gamma \in(2-\alpha-\beta, 1), p \in[1, \infty)$, is a normed linear space over $\mathbf{C}$ endowed with the norm $\|\cdot\|_{\mathscr{D}_{A}(\gamma, p)}$. This completes the proof.

REMARK 6.4. We stress that $\{0\} \cup\left[\mathscr{D}_{A}(\gamma, p) \backslash A 0\right], \quad \gamma \in(2-\alpha-\beta, 1), \quad p \in$ $[1, \infty]$, is, in general, only a normed linear space over $\mathbf{C}$ endowed with the norm $\|\cdot\|_{\mathscr{D}_{A}(\gamma, p)}$ of $\mathscr{D}_{A}(\gamma, p)$, but it may not be a Banach space. For, due to $A^{-1} \in \mathscr{L}(X)$, the linear subspace $A 0$ is closed $\operatorname{on}^{2} X$ and hence $\mathscr{D}_{A}(\gamma, p) \backslash A 0$ is, in general, only an open subset of $\mathscr{D}_{A}(\gamma, p)$.

REMARK 6.5. Observe that from the second in (6.8) and the property $\left[X_{A}^{\sigma, p} \cap A 0\right]=\{0\}, \sigma \in(0,1), p \in[1, \infty]$, we get $X_{A}^{\gamma, \infty} \subseteq\{0\} \cup\left[D_{A}(\gamma, \infty) \backslash A 0\right], \gamma \in$ $(0,1)$. Therefore, in the special case $\beta=1$, this latter inclusion combined with the second in (6.9) yields $X_{A}^{\gamma, \infty}=\{0\} \cup\left[\mathscr{D}_{A}(\gamma, \infty) \backslash A 0\right]$ in the sense of equivalence of the respective norms.

We can now prove our main theorems.

THEOREM 6.6. Let $A$ be a $m$. l. operator satisfying $(\mathrm{H} 1)$ with $\alpha \in(1 / 2,1]$ and $\beta \in(1 / 2, \alpha]$. Then, for every $\theta \in \mathbf{C}$ such that $\Re \mathrm{e} \theta \in(1-\beta, \beta)$ it holds that

$$
\mathscr{D}(A) \hookrightarrow X_{A}^{\Re \mathrm{e} \theta, 1} \hookrightarrow \mathscr{D}\left((-A)^{\theta}\right), \quad X_{A}^{\Re \mathrm{e} \theta, 1} \subseteq\{0\} \cup\left[\mathscr{D}\left((-A)^{\theta}\right) \backslash A 0\right] .
$$

Proof. We introduce the single-valued linear operator

$$
\left\{\begin{array}{l}
{\left[(-A)^{\theta}\right]^{\circ}=-\frac{1}{2 \pi i} \int_{\Gamma}(-\lambda)^{\theta-1} A^{\circ}(\lambda I-A)^{-1} \mathrm{~d} \lambda, \quad \Re \mathrm{e} \theta \in(1-\beta, \beta),} \\
\mathscr{D}\left(\left[(-A)^{\theta}\right]^{\circ}\right)=X_{A}^{\Re \mathrm{e} \theta, 1}
\end{array}\right.
$$

\footnotetext{
${ }^{2}$ In fact, if $\left\{x_{n}\right\}_{n \in \mathbf{N}} \subset A 0$ is such that $x_{n} \rightarrow x$ in $X$, then from $A^{-1} \in \mathcal{L}(X)$ it follows that $0=A^{-1} x_{n} \rightarrow A^{-1} x$. Hence, $A^{-1} x=0$ or, equivalently, $x \in A 0$.
} 
where $\mathscr{D}\left(\left[(-A)^{\theta}\right]^{\circ}\right)$ is endowed with the graph norm $\|x\|_{\mathscr{D}\left(\left[(-A)^{\theta}\right]^{\circ}\right)}=\|x\|_{X}+$ $\left\|\left[(-A)^{\theta}\right]^{\circ} x\right\|_{X}$. We shall prove that, if $x \in X_{A}^{\Re e} \theta, 1$, then $x=(-A)^{-\theta} y$ where $y=\left[(-A)^{\theta}\right]^{\circ} x$, yielding $x \in \mathscr{R}\left((-A)^{-\theta}\right)=\mathscr{D}\left((-A)^{\theta}\right)$. The embeddings and the inclusion in (6.24) will then follow from Remarks 4.2 and 4.5 , and inequality (6.31) below. We first show that $\left[(-A)^{\theta}\right]^{\circ}$ is well defined on $X_{A}^{\Re e} \theta, 1$. To this purpose, using Cauchy's theorem, we deform the infinite branches $\Gamma_{+}$and $\Gamma_{-}$ of $\Gamma$ into the upper and lower sides of the positive real axis, respectively. That is $\Gamma_{ \pm} \rightarrow \tilde{\Gamma}_{ \pm}$, where $\tilde{\Gamma}_{ \pm}=\{z \in \mathbf{C}: z=\xi \pm \mathrm{i} 0, \xi \in(0, \infty)\}$. Therefore, since for $\Re \mathrm{e} \theta \in(1-\beta, \beta)$ we have $(-\lambda)^{\theta-1}=\mathrm{e}^{(\theta-1) \log (-\lambda)}=|\lambda|^{\theta-1} \mathrm{e}^{\mp(\theta-1) \pi i}, \quad \lambda \in \tilde{\Gamma}_{ \pm}$, we obtain

$$
\begin{aligned}
{\left[(-A)^{\theta}\right]^{\circ} x=} & -\frac{1}{2 \pi i} \int_{0}^{\infty} \xi^{\theta-1} \mathrm{e}^{-(\theta-1) \pi i} A^{\circ}(\xi I-A)^{-1} x \mathrm{~d} \xi \\
& -\frac{1}{2 \pi i} \int_{\infty}^{0} \xi^{\theta-1} \mathrm{e}^{(\theta-1) \pi i} A^{\circ}(\xi I-A)^{-1} x \mathrm{~d} \xi \\
= & \frac{\mathrm{e}^{(\theta-1) \pi i}-\mathrm{e}^{-(\theta-1) \pi i}}{2 \pi i} \int_{0}^{\infty} \xi^{\theta-1} A^{\circ}(\xi I-A)^{-1} x \mathrm{~d} \xi \\
= & -\frac{\sin (\theta \pi)}{\pi} \int_{0}^{\infty} \xi^{\theta-1} A^{\circ}(\xi I-A)^{-1} x \mathrm{~d} \xi .
\end{aligned}
$$

As a consequence

$$
\left\|\left[(-A)^{\theta}\right]^{\circ} x\right\|_{X} \leq \pi^{-1} \mathrm{e}^{|\Im \mathrm{m} \theta| \pi}[x]_{X_{A}^{\Re e} \theta, 1}<\infty, \quad \forall x \in X_{A}^{\Re \mathrm{e} \theta, 1} .
$$

Now, the map $\lambda \in \rho(A) \rightarrow(-\lambda)^{\theta-1} A^{\circ}(\lambda I-A)^{-1} \in \mathscr{L}(X)$ being holomorphic, we replace the contour $\Gamma$ in $(6.25)$ with the contour $\Gamma^{\prime}$ parametrized by $\mu=$ $-c^{\prime}(|\eta|+1)^{\alpha}+i \eta, \quad \eta \in \mathbf{R}, \quad c^{\prime} \in(0, c)$. Then, using (2.7) and (3.1), for every $x \in X_{A}^{\Re \mathrm{e} \theta, 1}, \Re \mathrm{e} \theta \in(1-\beta, \beta)$, we find

$$
\begin{aligned}
&(-A)^{-\theta}\left[(-A)^{\theta}\right]^{\circ} x \\
&=-\left(\frac{1}{2 \pi i}\right)^{2} \int_{\Gamma}(-\lambda)^{-\theta}\left[\int_{\Gamma^{\prime}}(-\mu)^{\theta-1}(\lambda I-A)^{-1} A^{\circ}(\mu I-A)^{-1} x \mathrm{~d} \mu\right] \mathrm{d} \lambda \\
&=-\left(\frac{1}{2 \pi i}\right)^{2} \int_{\Gamma}(-\lambda)^{-\theta} A^{\circ}(\lambda I-A)^{-1}\left[\left(\int_{\Gamma^{\prime}}(-\mu)^{\theta-1}(\mu-\lambda)^{-1} \mathrm{~d} \mu\right) x\right] \mathrm{d} \lambda \\
&-\left(\frac{1}{2 \pi i}\right)^{2} \int_{\Gamma^{\prime}}(-\mu)^{\theta-1} A^{\circ}(\mu I-A)^{-1}\left[\left(\int_{\Gamma}(-\lambda)^{-\theta}(\lambda-\mu)^{-1} \mathrm{~d} \lambda\right) x\right] \mathrm{d} \mu .
\end{aligned}
$$


After having enclosed $\Gamma$ and $\Gamma^{\prime}$ on the left with an arc of the circle $\left\{z \in \mathbf{C}:\left|z+c^{\prime}\right|=R\right\}, R>c-c^{\prime}$, we apply the residue theorem and we let $R$ go to infinity. Since $\Gamma^{\prime}$ lies to the right of $\Gamma$, the same computations as in [32, pp. 547, 548] yield $\int_{\Gamma^{\prime}}(-\mu)^{\theta-1}(\mu-\lambda)^{-1} \mathrm{~d} \mu=2 \pi i(-\lambda)^{\theta-1}$ and $\int_{\Gamma}(-\lambda)^{-\theta}(\lambda-\mu)^{-1} \mathrm{~d} \lambda=0$. Hence, from (6.28) we get

$$
(-A)^{-\theta}\left[(-A)^{\theta}\right]^{\circ} x=\frac{1}{2 \pi i} \int_{\Gamma} \lambda^{-1} A^{\circ}(\lambda I-A)^{-1} x \mathrm{~d} \lambda, \quad \forall x \in X_{A}^{\Re \mathrm{e} \theta, 1} .
$$

Since $x \in X_{A}^{\Re e} \theta, 1 \hookrightarrow(X, \mathscr{D}(A))_{\Re \mathrm{e} \theta, 1}$, 凡e $\theta \in(1-\beta, \beta)$, the right-hand side of $(6.29)$ is precisely the element $w$ defined by $(5.7)$ with $(\gamma, p)=(\Re \mathrm{e} \theta, 1)$. Therefore, the same proof as that in Proposition 5.2 shows $w-x \in\left[X_{A}^{\Re e} \theta+\beta-1, \infty \cap A 0\right]=\{0\}$ and (6.28) reduces to

$$
(-A)^{-\theta}\left[(-A)^{\theta}\right]^{\circ} x=x, \quad \forall x \in X_{A}^{\Re \mathrm{e} \theta, 1}
$$

Then $x \in \mathscr{R}\left((-A)^{-\theta}\right)=\mathscr{D}\left((-A)^{\theta}\right)$ for every $x \in X_{A}^{\Re \mathrm{e} \theta, 1}$, Re $\theta \in(1-\beta, \beta)$, showing that the inclusion $X_{A}^{\Re \mathrm{e} \theta, 1} \subseteq \mathscr{D}\left((-A)^{\theta}\right)$, $\Re \mathrm{e} \theta \in(1-\beta, \beta)$, holds. Also, (6.30) implies $\left[(-A)^{\theta}\right]^{\circ} x \in(-A)^{\theta} x, x \in X_{A}^{\Re \mathrm{e} \theta, 1}$, meaning that $\left[(-A)^{\theta}\right]^{\circ}$ is a section of $\left.(-A)^{\theta}\right|_{X_{A}^{\Re e} \theta, 1}$. Thus, for every $x \in X_{A}^{\Re e \theta, 1}$, from (6.27) we deduce

$$
\|x\|_{\mathscr{D}\left((-A)^{\theta}\right)}=\inf _{y \in(-A)^{\theta} x}\|y\|_{X} \leq\left\|\left[(-A)^{\theta}\right]^{\circ} x\right\|_{X} \leq \pi^{-1} \mathrm{e}^{|\Im \mathrm{m} \theta|}\|x\|_{X_{A}^{\text {凡e } \theta, 1}}
$$

completing the proof of $X_{A}^{\Re e} \theta, 1 \hookrightarrow \mathscr{D}\left((-A)^{\theta}\right)$, 凡e $\theta \in(1-\beta, \beta)$. Finally (6.24) follows from $\mathscr{D}(A) \hookrightarrow X_{A}^{\varphi, p}, \varphi \in(0, \beta), p \in[1, \infty]$ and $\left[X_{A}^{\varphi, p} \cap A 0\right]=\{0\}, \varphi \in(0,1)$, $p \in[1, \infty]$.

Remark 6.7. Due to (6.26) and Remark 4.1, if $A$ is a single-valued densely defined linear operator satisfying $(\mathrm{H} 1)$ with $\beta=1$, then the fractional power $(-A)^{\theta}, \Re \mathrm{e} \theta \in(0,1)$, is just the closure of $\left[(-A)^{\theta}\right]^{\circ}$ (cf. [24, Section 2] and [35, Subsection 1.15.1]).

REMARK 6.8. The change of variable $\xi=t^{-1}$ in the right-hand side of (6.26) yields

$$
\left[(-A)^{\theta}\right]^{\circ} x=\frac{\sin (\theta \pi)}{\pi} \int_{0}^{\infty} t^{-\theta}(-A)_{t} x \mathrm{~d} t, \quad(-A)_{t}:=\left[I-(I-t A)^{-1}\right] / t,
$$

showing that $\left[(-A)^{\theta}\right]^{\circ}$ coincides with the operator $\mathfrak{F}_{\theta}(-A)$ in $[1$, p. 166]. Assuming $\beta=1$ and considering only fractional powers with real exponents, in [1] it is proved $\mathscr{D}(A) \subseteq \mathscr{D}\left(\mathfrak{F}_{\theta}(-A)\right) \subseteq \mathscr{D}\left((-A)^{\theta}\right), \quad \theta \in(0,1)$, where $\mathscr{D}\left(\mathfrak{F}_{\theta}(-A)\right)=$ 
$\left\{x: \int_{0}^{\infty} t^{-\theta}\left\|A_{t} x\right\|_{X} \mathrm{~d} t<\infty\right\}$. The transformation $t^{-1}=\xi$ thus shows that $\mathscr{D}\left(\mathfrak{F}_{\theta}(-A)\right)$ is just $X_{A}^{\theta, 1}$, and the result in [1] may be restated as $\mathscr{D}(A) \subseteq X_{A}^{\theta, 1} \subseteq$ $\mathscr{D}\left((-A)^{\theta}\right), \theta \in(0,1)$. Of course, even in a weaker form, this inclusion agrees with (6.24) for $\beta=1$ and $\theta \in \mathbf{R}$. The main problem in [1] is that $\mathscr{D}\left(\mathfrak{F}_{\theta}(-A)\right)$ is not characterized in terms of interpolation spaces. We mean, differently from what follows from Proposition 4.3 with $\beta=1$, in [1] the domain $\mathscr{D}\left(\mathfrak{F}_{\theta}(-A)\right)=X_{A}^{\theta, 1}$ is not recovered to be just the interpolation space $(X, \mathscr{D}(A))_{\theta, 1}$.

THeOREM 6.9. Let $A$ be a m. $l$. operator satisfying (H1) with $\alpha+\beta>1$. Then, for every $\theta \in \mathbf{C}$ such that $\Re \mathrm{e} \theta \in(2-\alpha-\beta, 1)$ it holds that

$$
\{0\} \cup\left[\mathscr{D}\left((-A)^{\theta}\right) \backslash A 0\right] \hookrightarrow X_{A}^{(\alpha+\beta+\Re \mathrm{e} \theta-2) / \alpha, \infty} .
$$

Here $\{0\} \cup\left[\mathscr{D}\left((-A)^{\theta}\right) \backslash A 0\right]$ is a normed linear space over $\mathbf{C}$ with the norm $\|\cdot\|_{\mathscr{D}\left((-A)^{\theta}\right)}$.

Proof. Due to the second embedding in (6.9) it suffices to show that $\mathscr{D}\left((-A)^{\theta}\right) \hookrightarrow \mathscr{D}_{A}(\Re \mathrm{e} \theta, \infty), \quad \Re \mathrm{e} \theta \in(2-\alpha-\beta, 1)$. Let $x \in \mathscr{D}\left((-A)^{\theta}\right)$ and take $y \in(-A)^{\theta} x$. Then, since $1-\beta \leq 2-\alpha-\beta$ and $x=(-A)^{-\theta} y$, using (3.21) and (3.23) we obtain

$$
\left\|\left[(-A)^{1}\right]^{\circ} \mathrm{e}^{t A} x\right\|_{X}=\left\|\left[(-A)^{1-\theta}\right]^{\circ} \mathrm{e}^{t A} y\right\|_{X} \leq \tilde{c}_{\alpha, \beta, 1-\theta} t^{(\beta+\Re \mathrm{e} \theta-2) / \alpha}\|y\|_{X} .
$$

Estimate (6.33) implies

$$
[x]_{\mathscr{D}_{A}(\Re \mathrm{e} \theta, \infty)} \leq c_{\alpha, \beta, 1-\theta}\|y\|_{X}, \quad \forall y \in(-A)^{\theta} x .
$$

In addition, if $x \in \mathscr{D}\left((-A)^{\theta}\right)$, then

$$
\|x\|_{X}=\left\|(-A)^{-\theta} y\right\|_{X} \leq\left\|(-A)^{-\theta}\right\|_{\mathscr{L}(X)}\|y\|_{X}, \quad \forall y \in(-A)^{\theta} x .
$$

Finally, taking the infimum with respect to $y \in(-A)^{\theta} x$, from (6.34) and (6.35) we get

$$
\|x\|_{\mathscr{D}_{A}(\Re \mathrm{e} \theta, \infty)}=\|x\|_{X}+[x]_{\mathscr{D}_{A}(\Re \mathrm{e} \theta, \infty)} \leq\left(\left\|(-A)^{-\theta}\right\|_{\mathscr{L}(X)}+c_{\alpha, \beta, 1-\theta}\|x\|_{\mathscr{D}\left((-A)^{\theta}\right)},\right.
$$

proving $\mathscr{D}\left((-A)^{\theta}\right) \hookrightarrow \mathscr{D}_{A}(\Re \mathrm{e} \theta, \infty), \Re \mathrm{e} \theta \in(2-\alpha-\beta, 1)$. Finally, the same reasonings made below (6.20), but with $\{0\} \cup\left[\mathscr{D}_{A}(\gamma, \infty) \backslash A 0\right]$ being replaced with $\{0\} \cup\left[\mathscr{D}\left((-A)^{\theta}\right) \backslash A 0\right]$, show that $\{0\} \cup\left[\mathscr{D}\left((-A)^{\theta}\right) \backslash A 0\right]$, $\Re \mathrm{e} \theta \in(2-\alpha-\beta, 1)$, is a normed linear space over $\mathbf{C}$ endowed with the norm $\|\cdot\|_{\mathscr{D}\left((-A)^{\theta}\right)}$. This completes the proof. 
The following Theorem 6.10 generalizes (4.26) to multivalued linear operators satisfying (H1) and having not necessarily dense domain.

THEOREM 6.10. Let $A$ be a $m$. l. operator satisfying $(\mathrm{H} 1)$ with $\alpha \in(2 / 3,1]$ and $\beta \in(1-\alpha / 2, \alpha]$. Then, for every $\theta \in \mathbf{C}$ such that $\Re \mathrm{e} \theta \in(2-\alpha-\beta, \beta)$ it holds that

$$
\mathscr{D}(A) \hookrightarrow X_{A}^{\Re \mathrm{e} \theta, 1} \hookrightarrow\{0\} \cup\left[\mathscr{D}\left((-A)^{\theta}\right) \backslash A 0\right] \hookrightarrow X_{A}^{(\alpha+\beta+\Re \mathrm{e} \theta-2) / \alpha, \infty}
$$

Here $\{0\} \cup\left[\mathscr{D}\left((-A)^{\theta}\right) \backslash A 0\right]$ is a normed linear space over $\mathbf{C}$ with the norm $\|\cdot\|_{\mathscr{D}\left((-A)^{\theta}\right)}$.

Proof. First, our assumptions on $\alpha$ and $\beta$ imply $\beta \in(1 / 2, \alpha]$ and $\alpha+\beta>$ $1+\alpha / 2>1$, so that the assumptions of Theorems 6.6 and 6.9 are satisfied. Moreover, since $\beta>1-\alpha / 2$ and $1-\beta \leq 2-\alpha-\beta$, the choice $\Re$ e $\theta \in$ $(2-\alpha-\beta, \beta)$ makes sense and satisfy the requirements for $\Re \mathrm{e} \theta$ in both the quoted theorems. Therefore, (6.36) follows from (6.24) and (6.32), where the inclusion in (6.24) now becomes just an embedding due to (6.31).

Remark 6.11. Of course, if $A$ is single-valued, densely defined, and $\beta=1$, then (6.36) coincides with embedding (4.26) proved in [24], [25] and [35]. Moreover, if $A$ is multivalued and $\beta=1$, then (6.36) refines the result in [1] mentioned in Remark 6.8 .

\section{On the Behaviour of $\mathrm{e}^{t A}$ with Respect to $\mathscr{D}\left((-A)^{\gamma}\right)$}

Here we study the behaviour of the operators $\left[(-A)^{\theta}\right]^{\circ} \mathrm{e}^{t A}, \Re \mathrm{e} \theta \geq 0, t>0$, defined by (3.19) with respect to the domains $\mathscr{D}\left((-A)^{\gamma}\right)$, $\Re$ e $\gamma>1-\beta$, of the fractional powers $(-A)^{\gamma}$ of $-A$. Throughout this section $A$ will be a m. 1 . operator in $X$ satisfying (H1).

Lemma 7.1. Let $\left[(-A)^{\gamma}\right]^{\circ}$, $\Re \mathrm{e} \gamma \in(1-\beta, \beta), \beta \in(1 / 2, \alpha], \alpha \in(1 / 2,1]$, be the operator defined by (6.25), and let $\left[(-A)^{\theta}\right]^{\circ} \mathrm{e}^{t A}$, $\Re \mathrm{e} \theta \geq 0, t>0$, be the operators defined by (3.19). Then, for every $x \in X$ it holds that

$$
\left[(-A)^{\gamma}\right]^{\circ}\left(\left[(-A)^{\theta}\right]^{\circ} \mathrm{e}^{t A} x\right)=\left[(-A)^{\gamma+\theta}\right]^{\circ} \mathrm{e}^{t A} x
$$

Proof. Let $x \in X$ and $\Re$ e $\gamma \in(1-\beta, \beta)$. First, from [8, Lemma 3.1] and Remark 4.5 we have $\left[(-A)^{\theta}\right]^{\circ} \mathrm{e}^{t A} x \in \mathscr{D}(A) \hookrightarrow X_{A}^{\Re \mathrm{e} \gamma, 1}$ and we can apply $\left[(-A)^{\gamma}\right]^{\circ}$ 
to $\left[(-A)^{\theta}\right]^{\circ} \mathrm{e}^{t A} x$. Replacing the contour $\Gamma$ in (3.19) with the contour $\Gamma^{\prime}$ defined in the proof of Theorem 6.6 and using (2.8), for every $t>0$ we find

$$
\begin{aligned}
{\left[(-A)^{\gamma}\right]^{\circ}\left(\left[(-A)^{\theta}\right]^{\circ} \mathrm{e}^{t A} x\right) } & \\
= & -\left(\frac{1}{2 \pi i}\right)^{2} \int_{\Gamma}(-\lambda)^{\gamma-1}\left[\int_{\Gamma^{\prime}}(-\mu)^{\theta} \mathrm{e}^{t \mu} A^{\circ}(\lambda I-A)^{-1}(\mu I-A)^{-1} x \mathrm{~d} \mu\right] \mathrm{d} \lambda \\
= & -\left(\frac{1}{2 \pi i}\right)^{2} \int_{\Gamma}(-\lambda)^{\gamma-1} A^{\circ}(\lambda I-A)^{-1}\left[\left(\int_{\Gamma^{\prime}}(-\mu)^{\theta} \mathrm{e}^{t \mu}(\mu-\lambda)^{-1} \mathrm{~d} \mu\right) x\right] \mathrm{d} \lambda \\
& -\left(\frac{1}{2 \pi i}\right)^{2} \int_{\Gamma^{\prime}}(-\mu)^{\theta} \mathrm{e}^{t \mu} A^{\circ}(\mu I-A)^{-1}\left[\left(\int_{\Gamma}(-\lambda)^{\gamma-1}(\lambda-\mu)^{-1} \mathrm{~d} \lambda\right) x\right] \mathrm{d} \mu .
\end{aligned}
$$

Since $\Gamma^{\prime}$ lies to the right of $\Gamma$, the residue theorem implies $\int_{\Gamma^{\prime}}(-\mu)^{\theta} \mathrm{e}^{t \mu}(\mu-\lambda)^{-1} \mathrm{~d} \mu$ $=2 \pi i(-\lambda)^{\theta} \mathrm{e}^{t \lambda}$ and $\int_{\Gamma}(-\lambda)^{\gamma-1}(\lambda-\mu)^{-1} \mathrm{~d} \lambda=0$. Therefore

$$
\begin{aligned}
{\left[(-A)^{\gamma}\right]^{\circ}\left(\left[(-A)^{\theta}\right]^{\circ} \mathrm{e}^{t A} x\right) } & =-\frac{1}{2 \pi i} \int_{\Gamma}(-\lambda)^{\gamma+\theta-1} \mathrm{e}^{t \lambda} A^{\circ}(\lambda I-A)^{-1} x \mathrm{~d} \lambda \\
& =-\frac{1}{2 \pi i} \int_{\Gamma}(-\lambda)^{\gamma+\theta-1} \mathrm{e}^{t \lambda}\left[\lambda(\lambda I-A)^{-1}-I\right] x \mathrm{~d} \lambda \\
& =\frac{1}{2 \pi i} \int_{\Gamma}(-\lambda)^{\gamma+\theta} \mathrm{e}^{t \lambda}(\lambda I-A)^{-1} x \mathrm{~d} \lambda .
\end{aligned}
$$

Here in the latter equality we have used $\int_{\Gamma}(-\lambda)^{\gamma+\theta-1} \mathrm{e}^{t \lambda} \mathrm{d} \lambda=0$ for every $t>0$, which follows from the Cauchy's formula after having enclosed $\Gamma$ on the left with an arc of the circle $\{z \in \mathbf{C}:|z+c|=R\}, R>0$, and then letting $R$ to infinity. The right-hand side of (7.2) being precisely $\left[(-A)^{\gamma+\theta}\right]^{\circ} \mathrm{e}^{t A} x$, the proof is complete.

REMARK 7.2. In particular, (7.1) with $\theta=0$ shows that at least for $\Re$ e $\gamma \in$ $(1-\beta, \beta)$ the operator $\left[(-A)^{\gamma}\right]^{\circ} \mathrm{e}^{t A}$ defined by (3.19) is just the product of the section $\left[(-A)^{\gamma}\right]^{\circ}$ of $\left.(-A)^{\gamma}\right|_{X_{A}^{\Re e} \gamma, 1}$ with the semigroup $\mathrm{e}^{t A}$.

Proposition 7.3. Let $\alpha, \beta, \gamma$ and $\theta$ be as in Lemma 7.1. Then, there exists $a$ positive constant $c_{25}=c_{25}(\alpha, \beta, \gamma, \theta)$ such that the following estimate holds

$$
\left\|\left[(-A)^{\theta}\right]^{\circ} \mathrm{e}^{t A}\right\|_{\mathscr{L}\left(X ; \mathscr{D}\left((-A)^{\gamma}\right)\right)} \leq c_{25} t^{(\beta-\Re \mathrm{e} \gamma-\Re \mathrm{e} \theta-1) / \alpha}, \quad \forall t>0 .
$$

Proof. Let $x \in X$. Then, since $\left[(-A)^{\theta}\right]^{\circ} \mathrm{e}^{t A} x \in \mathscr{D}(A) \hookrightarrow X_{A}^{\Re \mathrm{e} \gamma, 1} \hookrightarrow \mathscr{D}\left((-A)^{\gamma}\right)$, $t>0$, $\Re \mathrm{e} \theta \geq 0, \Re \mathrm{e} \gamma \in(1-\beta, \beta)$, from the fact that $\left[(-A)^{\gamma}\right]^{\circ}$ is a section of 
$\left.(-A)^{\gamma}\right|_{X_{A}^{\Re e} \gamma, 1}$ using identity (7.1) and estimates (3.23) we get

$$
\left\|\left[(-A)^{\theta}\right]^{\circ} \mathrm{e}^{t A} x\right\|_{\mathscr{D}\left((-A)^{\gamma}\right)} \leq\left\|\left[(-A)^{\gamma+\theta}\right]^{\circ} \mathrm{e}^{t A} x\right\|_{X} \leq \tilde{c}_{\alpha, \beta, \gamma+\theta} t^{(\beta-\Re \mathrm{e} \gamma-\Re \mathrm{e} \theta-1) / \alpha}\|x\|_{X} .
$$

The proof is complete.

As a corollary we have that, if $t$ is bounded away from zero, then for every $\sigma \in(0,1)$ the operator function $t \rightarrow\left[(-A)^{\theta}\right]^{\circ} \mathrm{e}^{t A}$ is $\sigma$-Hölder continuous with values in $\mathscr{L}\left(X ; \mathscr{D}\left((-A)^{\gamma}\right)\right)$.

COROLlary 7.4. Let $\alpha, \beta, \gamma$ and $\theta$ be as in Lemma 7.1. Then, for every $\sigma \in(0,1)$ and $0<s<t$ the following estimate holds where $c_{26}=\sigma^{-1} c_{25}$ :

$\left\|\left[(-A)^{\theta}\right]^{\circ} \mathrm{e}^{t A}-\left[(-A)^{\theta}\right]^{\circ} \mathrm{e}^{s A}\right\|_{\mathscr{L}\left(X ; \mathscr{D}\left((-A)^{\gamma}\right)\right)} \leq c_{26} S^{(\alpha+\beta-\Re \mathrm{e} \gamma-\Re \mathrm{e} \theta-2-\alpha \sigma) / \alpha}(t-s)^{\sigma}$.

Proof. It is shown in $[8$, formula (22)] that

$$
\left[(-A)^{\theta}\right]^{\circ} \mathrm{e}^{t A}-\left[(-A)^{\theta}\right]^{\circ} \mathrm{e}^{s A}=-\int_{s}^{t}\left[(-A)^{\theta+1}\right]^{\circ} \mathrm{e}^{\xi A} \mathrm{~d} \xi, \quad 0<s<t .
$$

Consequently, for every $\sigma \in(0,1)$, using (7.3) with $\theta$ being replaced by $\theta+1$ and the well-known inequality $t^{\sigma}-s^{\sigma} \leq(t-s)^{\sigma}$, from (7.5) it follows that

$$
\begin{aligned}
\left\|\left[(-A)^{\theta}\right]^{\circ} \mathrm{e}^{t A}-\left[(-A)^{\theta}\right]^{\circ} \mathrm{e}^{s A}\right\|_{\mathscr{L}\left(X ; \mathscr{D}\left((-A)^{\gamma}\right)\right)} & \leq c_{25} \int_{s}^{t} \xi^{(\beta-\Re \mathrm{e} \gamma-\Re \mathrm{e} \theta-2) / \alpha} \mathrm{d} \xi \\
& \leq c_{25} S^{(\alpha+\beta-\Re \mathrm{e} \gamma-\Re \mathrm{e} \theta-2-\alpha \sigma) / \alpha} \int_{s}^{t} \xi^{\sigma-1} \mathrm{~d} \xi \\
& \leq \sigma^{-1} c_{25} S^{(\alpha+\beta-\Re \mathrm{e} \gamma-\Re \mathrm{e} \theta-2-\alpha \sigma) / \alpha}(t-s)^{\sigma} .
\end{aligned}
$$

This completes the proof of (7.4).

Remark 7.5. Proposition 7.3 and Corollary 7.4 show that, if $\alpha \in(1 / 2,1]$, $\beta \in(1 / 2, \alpha]$, then the operators $\left[(-A)^{\theta}\right]^{\circ} \mathrm{e}^{t A}$, considered from $X$ to $\mathscr{D}\left((-A)^{\gamma}\right)$, $\Re$ e $\gamma \in(1-\beta, \beta)$, behave like when they are considered as operators from $X$ to the interpolation spaces $(X, \mathscr{D}(A))_{\gamma, p}, \gamma \in(0,1), p \in[1, \infty]$. Indeed (cf. [8, formulae (19) and (21)]), for every $0<s<t$ the following estimate hold with $c_{27}=c_{27}(\alpha, \beta, \gamma, \theta, p)>0$ and $c_{28}=\sigma^{-1} c_{27}$ :

$$
\begin{aligned}
& \left\|\left[(-A)^{\theta}\right]^{\circ} \mathrm{e}^{t A}\right\|_{\mathscr{L}\left(X ;(X, \mathscr{D}(A))_{\gamma, p}\right)} \leq c_{27} t^{(\beta-\gamma-\Re \mathrm{e} \theta-1) / \alpha}, \\
& \left\|\left[(-A)^{\theta}\right]^{\circ} \mathrm{e}^{t A}-\left[(-A)^{\theta}\right]^{\circ} \mathrm{e}^{s A}\right\|_{\mathscr{L}\left(X ;(X, \mathscr{D}(A))_{\gamma, p}\right)} \leq c_{28} s^{(\alpha+\beta-\gamma-\Re \mathrm{e} \theta-2-\alpha \sigma) / \alpha}(t-s)^{\sigma} .
\end{aligned}
$$


We stress that (7.6) is derived in [8] by means of interpolation techniques that can not be employed for the derivation of (7.3). For, due to (3.11) and Proposition 3.3, the domains $\mathscr{D}\left((-A)^{\gamma}\right)$, $\Re \mathrm{e} \gamma \in(1-\beta, \beta)$, are in general only intermediate spaces between $X$ and $\mathscr{D}(A)$, but may be not real interpolation spaces. Here, the key role in the derivation of (7.3) is played by Lemma 7.1 and the property of $\left[(-A)^{\gamma}\right]^{\circ}$ to be a section of $\left.(-A)^{\gamma}\right|_{X_{A}^{\Re e} \gamma, 1}$.

Due to Proposition 7.3, Corollary 7.4 and Remark 7.5 we can extend the results in $\left[8\right.$, Section 4], replacing there the interpolation spaces $(X, \mathscr{D}(A))_{\gamma, p}$, $\gamma \in(0,1), p \in[1, \infty]$ with the domains $\mathscr{D}\left((-A)^{\gamma}\right)$, $\Re$ e $\gamma \in(1-\beta, \beta)$, of the fractional powers $(-A)^{\gamma}$ of $-A$. We warn that this is not a trivial extension, for, in general, the space $(X, \mathscr{D}(A))_{\Re e \gamma, p}$ and $\mathscr{D}\left((-A)^{\gamma}\right)$ are not comparable. At the best (cf. (1.2) and (4.2)), when $A$ is single-valued and $\beta=1$, it is only known that for $\Re \mathrm{e} \gamma \in(0,1)$ the spaces $(X, \mathscr{D}(A))_{\Re \mathrm{e} \gamma, p}, p \in(1, \infty)$, and $\mathscr{D}\left((-A)^{\gamma}\right)$ are intermediate spaces between $(X, \mathscr{D}(A))_{\Re \mathrm{e} \gamma, \infty}$ and $(X, \mathscr{D}(A))_{\Re \mathrm{e} \gamma, 1}$.

From now on, given a complex Banach space $\left(Z,\|\cdot\|_{Z}\right), C([0, T] ; Z)=$ $C^{0}([0, T] ; Z)$ and $C^{\delta}([0, T] ; Z), \delta \in(0,1), T>0$, denote, respectively, the spaces of all continuous and $\delta$-Hölder continuous functions from $[0, T]$ into $Z$ endowed with the usual norms

$$
\|g\|_{C^{\delta}([0, T] ; Z)}=\|g\|_{\delta, T ; Z}= \begin{cases}\sup _{t \in[0, T]}\|g(t)\|_{Z}, & \text { if } \delta=0, \\ \|g\|_{0, T ; Z}+|g|_{\delta, T ; Z}, & \text { if } \delta \in(0,1),\end{cases}
$$

where $\quad|g|_{\delta, T ; Z}=\sup _{0 \leq s<t \leq T}(t-s)^{-\delta}\|g(t)-g(s)\|_{Z} . \quad$ Moreover, we set $C^{1+\delta}([0, T] ; Z)=\left\{g \in C([0, T] ; Z): D_{t} g \in C^{\delta}([0, T] ; Z)\right\}, \delta \in[0,1)$, and $\|g\|_{1+\delta, T ; Z}$ $=\|g\|_{0, T ; Z}+\left\|D_{t} g\right\|_{\delta, T ; Z}$. Through the rest of the paper, given $\alpha \in(0,1], \beta \in(0, \alpha]$, $\gamma \in \mathbf{C}$ and $\sigma \in(0,1), c_{j}(T), j=29, \ldots$, denote positive nondecreasing functions of $T$ depending on $\alpha, \beta, \gamma$ and $\sigma$.

Lemma 7.6. Let $2 \alpha+2 \beta>3$. Then, for every $\Re \mathrm{e} \gamma \in(1-\beta, 2 \alpha+\beta-2)$ and $\sigma \in(0,(2 \alpha+\beta-\Re \mathrm{e} \gamma-2) / \alpha)$ the linear operator

$$
\left[Q_{1} g\right](t):=\int_{0}^{t} \mathrm{e}^{(t-\xi) A} g(\xi) \mathrm{d} \xi, \quad t \in[0, T],
$$

maps $C([0, T] ; X)$ into $C^{\sigma}\left([0, T] ; \mathscr{D}\left((-A)^{\gamma}\right)\right)$ and satisfies the estimate:

$$
\left\|Q_{1} g\right\|_{\sigma, T ; \mathscr{D}\left((-A)^{\gamma}\right)} \leq T^{(2 \alpha+\beta-\Re \mathrm{e} \gamma-2-\alpha \sigma) / \alpha} c_{29}(T)\|g\|_{0, T ; X} .
$$

Proof. Due to Proposition 7.3 and Corollary 7.4 the proof is the same as that of $[8$, Lemma 4.1]. To this purpose it suffices to replace in that proof 
$\gamma \in(0,2 \alpha+\beta-2), 2 \alpha+\beta>2$, and $(X, \mathscr{D}(A))_{\gamma, p}$ with $\Re \mathrm{e} \gamma \in(1-\beta, 2 \alpha+\beta-2)$, $2 \alpha+2 \beta>3$, and $\mathscr{D}\left((-A)^{\gamma}\right)$, respectively, and to use estimates (7.3) and (7.4) with $\theta=0$ in place of (7.6) and (7.7) with $\theta=0 . c_{29}(T)$ is given by [8, formula (27)], replacing there $\gamma$ and $c_{1}=c_{27}$ with $\Re$ e $\gamma$ and $c_{25}$.

Lemma 7.7. Let $\alpha+2 \beta>2$ and let $x \in \mathscr{D}\left((-A)^{\varphi}\right)$, $\Re \mathrm{e} \varphi \in(3-\alpha-2 \beta, 1]$. Then, for every $\Re \mathrm{e} \gamma \in(1-\beta, \alpha+\beta+\Re \mathrm{e} \varphi-2)$ and $\sigma \in(0,(\alpha+\beta-\Re \mathrm{e} \gamma+$ $\Re \mathrm{e} \varphi-2) / \alpha]$ it holds that $\mathrm{e}^{A} x \in C^{\sigma}\left([0, T] ; \mathscr{D}\left((-A)^{\gamma}\right)\right)$ with

$$
\left\|\mathrm{e}^{\cdot A} x\right\|_{\sigma, T ; \mathscr{D}\left((-A)^{\gamma}\right)} \leq c_{30}(T)\|x\|_{\mathscr{D}\left((-A)^{\varphi}\right)} .
$$

Proof. Let $x \in \mathscr{D}\left((-A)^{\varphi}\right), \Re \mathrm{e} \varphi \in(3-\alpha-2 \beta, 1] \subseteq(2(1-\beta), 1]$, and let $y \in$ $(-A)^{\varphi} x$ be arbitrary. Then, since $x=(-A)^{-\varphi} y$ and (cf. [12, Theorem 3.3]) $\mathrm{e}^{t A}$ is strongly continuous on $\mathscr{D}\left((-A)^{\varphi}\right)$ in the norm of $X$, using $(3.21)$ we find

$$
\mathrm{e}^{t A} x-x=-\int_{0}^{t}\left[(-A)^{1}\right]^{\circ} \mathrm{e}^{\xi A}(-A)^{-\varphi} y \mathrm{~d} \xi=-\int_{0}^{t}\left[(-A)^{1-\varphi}\right]^{\circ} \mathrm{e}^{\xi A} y \mathrm{~d} \xi
$$

Thus, when $\Re \mathrm{e} \gamma \in(1-\beta, \alpha+\beta+\Re \mathrm{e} \varphi-2) \subseteq(1-\beta, \beta+\Re \mathrm{e} \varphi-1) \subseteq(1-\beta, \beta)$, estimate (7.3) with $\theta=1-\varphi$ yields

$$
\left\|\mathrm{e}^{t A} x-x\right\|_{\mathscr{D}\left((-A)^{\gamma}\right)} \leq c_{25} \int_{0}^{t} \xi^{(\beta-\Re \mathrm{e} \gamma+\Re \mathrm{e} \varphi-2) / \alpha}\|y\|_{X} \mathrm{~d} \xi \leq \varrho^{-1} c_{25}\|y\|_{X} t^{\varrho},
$$

where $\varrho=(\alpha+\beta-\Re \mathrm{e} \gamma+\Re \mathrm{e} \varphi-2) / \alpha>0$. Passing to the infimum with respect to $y \in(-A)^{\varphi} x$, from (7.11) it follows that:

$$
\left\|\mathrm{e}^{t A} x-x\right\|_{\mathscr{D}\left((-A)^{\gamma}\right)} \leq \varrho^{-1} c_{25}\|x\|_{\mathscr{D}\left((-A)^{\varphi}\right)} t^{\varrho} .
$$

Now, from (3.12) with $\left(\theta_{1}, \theta_{2}\right)=(\varphi, \gamma)$ we have $\mathscr{D}\left((-A)^{\varphi}\right) \hookrightarrow \mathscr{D}\left((-A)^{\gamma}\right)$, so that, for every $t \in[0, T]$, from (7.12) we get

$$
\left\|\mathrm{e}^{t A} x\right\|_{\mathscr{D}\left((-A)^{\gamma}\right)} \leq\left\|\mathrm{e}^{t A} x-x\right\|_{\mathscr{D}\left((-A)^{\gamma}\right)}+\|x\|_{\mathscr{D}\left((-A)^{\gamma}\right)} \leq \tilde{c}_{30}(t)\|x\|_{\mathscr{D}\left((-A)^{\varphi}\right)},
$$

where $\tilde{c}_{30}(t)=\varrho^{-1} c_{25} t^{\varrho}+\left\|(-A)^{-(\varphi-\gamma)}\right\|_{\mathscr{L}(X)}$. Now, let $\sigma \in(0, \varrho]$ and ${ }^{3} \quad 0<s<$ $t \leq T$. Then, reasoning as in the derivation of (7.11), for every $y \in(-A)^{\varphi} x$ we obtain

$$
\left\|\mathrm{e}^{t A} x-\mathrm{e}^{s A} x\right\|_{\mathscr{D}\left((-A)^{\gamma}\right)} \leq \int_{s}^{t}\left\|\left[(-A)^{1-\varphi}\right]^{\circ} \mathrm{e}^{\xi A} y\right\|_{\mathscr{D}\left((-A)^{\gamma}\right)} \mathrm{d} \xi \leq \varrho^{-1} c_{25}\|y\|_{X}(t-s)^{\varrho} .
$$

\footnotetext{
$\overline{{ }^{3} \text { Since } \sigma \in(0, \varrho] \text { and } \mathrm{e}^{0 A}}=I$, the case $s=0$ follows from (7.12).
} 
Therefore, as in (7.12), from the latter inequality it follows that

$$
\left\|\mathrm{e}^{t A} x-\mathrm{e}^{s A} x\right\|_{\mathscr{D}\left((-A)^{\gamma}\right)} \leq \varrho^{-1} c_{25} T^{\varrho-\sigma}\|x\|_{\mathscr{D}\left((-A)^{\varphi}\right)}(t-s)^{\sigma} .
$$

From (7.13) and (7.14) we deduce (7.10) with $c_{30}(T)=\tilde{c}_{30}(T)+\varrho^{-1} c_{25} T^{\varrho-\sigma}$.

REMARK 7.8. A result similar to Lemma 7.7 is shown in [8, Lemma 4.3], but with $x \in \mathscr{D}\left((-A)^{\varphi}\right), \Re \mathrm{e} \varphi \in(3-\alpha-2 \beta, 1], \Re \mathrm{e} \gamma \in(1-\beta, \alpha+\beta+\Re \mathrm{e} \varphi-2)$ and $\mathscr{D}\left((-A)^{\gamma}\right)$ being replaced, respectively, by $x \in \mathscr{D}\left((-A)^{\varphi}\right) \cap X_{A}^{\varphi, 1}, \varphi \in(2-\alpha-\beta, \beta)$, $\gamma \in(0, \alpha+\beta+\varphi-2)$ and $(X, \mathscr{D}(A))_{\gamma, p}, p \in[1, \infty]$. In fact, at the time of [8], inclusion (6.24) and embedding (6.36) were not known. Now, with the result of this paper we can conclude that the intersection $\mathscr{D}\left((-A)^{\varphi}\right) \cap X_{A}^{\varphi, 1}, \varphi \in$ $(2-\alpha-\beta, \beta)$, in [8, Lemma 4.3] reduces to $X_{A}^{\varphi, 1}$. Consequently, all the results in $\left[8\right.$, Sections 5 and 6] can be restated replacing that intersection with $X_{A}^{\varphi, 1}$.

Remark 7.9. Since from Proposition 3.3 we have $\mathscr{D}(A) \hookrightarrow \mathscr{D}\left((-A)^{\varphi}\right)$, $\Re \mathrm{e} \varphi \in(1-\beta, \beta)$, if we want to ensure that the element $x$ in Lemma 7.7 may not belong to $\mathscr{D}(A)$, i.e. that is less regular, we have to take $\Re$ e $\varphi \in(3-\alpha-2 \beta, \beta)$. Of course, this can be made, but provided that the more restrictive assumption $\alpha+3 \beta>3$ is satisfied. When we shall consider applications in Section 8 it will be clearer the importance of being permitted to take $x$ in a space bigger than $\mathscr{D}(A)$ (cf. Theorems 8.1 and 8.2 with $x=u_{0}$ and $x=g_{0}$ ).

Lemma 7.10. Let $2 \alpha+2 \beta>3$. Then, for every $\mu \in((3-\alpha-2 \beta) / \alpha, 1)$, $\Re \mathrm{e} \gamma \in(1-\beta, \alpha \mu+\alpha+\beta-2)$ and $\sigma \in(0,(\alpha \mu+\alpha+\beta-\Re \mathrm{e} \gamma-2) / \alpha)$, the linear operator

$$
\left[Q_{2} f\right](t):=\mathrm{e}^{t A}[f(t)-f(0)], \quad t \in[0, T],
$$

maps $C^{\mu}([0, T] ; X)$ into $C^{\sigma}\left([0, T] ; \mathscr{D}\left((-A)^{\gamma}\right)\right)$ and satisfies the estimate:

$$
\left\|Q_{2} f\right\|_{\sigma, T ; \mathscr{D}\left((-A)^{\gamma}\right)} \leq T^{(\alpha \mu+\alpha+\beta-\Re \mathrm{e} \gamma-2-\alpha \sigma) / \alpha} c_{31}(T)|f|_{\mu, T ; X} .
$$

Proof. Due to Proposition 7.3 and Corollary 7.4 the proof is the same as that of $[8$, Lemma 4.4], except for replacing there $\mu \in((2-\alpha-\beta) / \alpha, 1)$, $2 \alpha+\beta>2, \gamma \in(0, \alpha \mu+\alpha+\beta-2)$ and $(X, \mathscr{D}(A))_{\gamma, p}$ with $\mu \in((3-\alpha-2 \beta) / \alpha, 1)$, $2 \alpha+2 \beta>3$, 凡e $\gamma \in(1-\beta, \alpha \mu+\alpha+\beta-2)$ and $\mathscr{D}\left((-A)^{\gamma}\right)$, respectively, and for using (7.3) and (7.4) with $\theta=0$ in place of (7.6) and (7.7) with $\theta=0 . c_{31}(T)$ is given by $\left[8\right.$, formula (44)] with $c_{1}$ being replaced by $c_{25}$. 
Lemma 7.11. Let $3 \alpha+2 \beta>4$. Then, for every $\mu \in((4-2 \alpha-2 \beta) / \alpha, 1)$, $\Re \mathrm{e} \gamma \in(1-\beta, \alpha \mu+2 \alpha+\beta-3)$ and $\sigma \in(0,(\alpha \mu+2 \alpha+\beta-\Re \mathrm{e} \gamma-3) / \alpha)$, the linear operator

$$
\left[Q_{3} f\right](t):=-\int_{0}^{t}\left[(-A)^{1}\right]^{\circ} \mathrm{e}^{(t-\xi) A}[f(\xi)-f(t)] \mathrm{d} \xi, \quad t \in[0, T],
$$

maps $C^{\mu}([0, T] ; X)$ into $C^{\sigma}\left([0, T] ; \mathscr{D}\left((-A)^{\gamma}\right)\right)$ and satisfies the estimate:

$$
\left\|Q_{3} f\right\|_{\sigma, T ; \mathscr{D}\left((-A)^{\gamma}\right)} \leq T^{(\alpha \mu+2 \alpha+\beta-\Re \mathrm{e} \gamma-3-\alpha \sigma) / \alpha} c_{32}(T)|f|_{\mu, T ; X} .
$$

Proof. Due to Proposition 7.3 and Corollary 7.4 the proof is the same as that of $\left[8\right.$, Lemma 4.5] where the notation $-A^{\circ} \mathrm{e}^{(t-\xi) A}$ is used for $\left[(-A)^{1}\right]^{\circ} \mathrm{e}^{(t-\xi) A}$. To this purpose it suffices to repeat the computations of that proof, except for replacing there $\mu \in((3-2 \alpha-\beta) / \alpha, 1), 3 \alpha+\beta>3, \gamma \in(0, \alpha \mu+2 \alpha+\beta-3)$ and $(X, \mathscr{D}(A))_{\gamma, p}$ with $\mu \in((4-2 \alpha-2 \beta) / \alpha, 1), 3 \alpha+2 \beta>4, \Re \mathrm{e} \gamma \in(1-\beta, \alpha \mu+2 \alpha+$ $\beta-3)$ and $\mathscr{D}\left((-A)^{\gamma}\right)$, respectively, and for using (7.3) and (7.4) with $\theta=1$ in place of (7.6) and (7.7) with $\theta=1$. Here $c_{32}(T)$ is given by [8, formula (53)], with $\gamma$ and $c_{1}$ being replaced with $\Re$ e $\gamma$ and $c_{25}$.

As we have seen in the proofs of Lemmas 7.6, 7.10 and 7.11, to extend [8, Lemmas 4.1, 4.4 and 4.5] to the case in which the space $C^{\sigma}\left([0, T] ; \mathscr{D}\left((-A)^{\gamma}\right)\right)$ is considered in place of $C^{\sigma}\left([0, T] ;(X, \mathscr{D}(A))_{\gamma, p}\right)$ we have to assume stronger assumptions on the pair $(\alpha, \beta)$ and to restrict the admissible subinterval of $(0,1)$ in which $\mu$ may varies. This is due to the fact that now we have to use estimates (7.3) and (7.4) which requires $\Re$ e $\gamma \in(1-\beta, \beta)$, instead of (7.6) and (7.7) where $\gamma$ may vary in the whole $(0,1)$. In particular, since in all the Lemmas 7.6, 7.10 and 7.11 the right extreme points of the admissible intervals for $\Re$ e $\gamma$ are less or equal than $\beta$, it is just the condition $\Re \mathrm{e} \gamma>1-\beta$ which forces us to strengthen the assumptions on the triplet $(\alpha, \beta, \mu)$ in the corresponding results of $[8]$.

\section{Applications}

Let $A$ be a m. 1 . operator in $X$ having domain $\mathscr{D}(A)$ and satisfying (H1). We shall use the results of Section 6 to provide maximal time regularity with respect to the domains of the fractional powers of $-A$ for the strict solution $u$ to the multivalued evolution problem

$$
D_{t} u(t) \in A u(t)+f(t), \quad t \in(0, T], \quad u(0)=u_{0},
$$


where $f \in C([0, T] ; X)$ and $u_{0} \in X$ are given. We recall that, according to [12, p. 53], a strict solution to $(8.1)$ is a function $u \in C^{1}((0, T] ; X)$ such that $u(t) \in \mathscr{D}(A)$ for every $t \in(0, T]$ and $u$ satisfies (8.1) where the initial condition is understood in the sense of the seminorm $p_{A}(\cdot)=\left\|A^{-1} \cdot\right\|_{X}$, that is $\lim _{t \rightarrow 0^{+}} p_{A}\left(u(t)-u_{0}\right)=0$. The strict solution is said to be a classical solution if $u \in C([0, T] ; X)$, that is $\lim _{t \rightarrow 0^{+}}\left\|u(t)-u_{0}\right\|_{X}=0$.

THEOREM 8.1. Let $2 \alpha+2 \beta>3$, let $u_{0} \in \mathscr{D}\left((-A)^{\varphi}\right)$, $\Re$ e $\varphi \in(3-\alpha-2 \beta, \alpha)$, and let $f \in C^{\mu}([0, T] ; X), \mu \in((2-\alpha-\beta) / \alpha, 1)$. Then, for every $\Re \mathrm{e} \gamma \in(1-\beta$, $\alpha+\beta+\Re \mathrm{e} \varphi-2)$ and $\sigma \in(0,(\alpha+\beta-\Re \mathrm{e} \gamma+\Re \mathrm{e} \varphi-2) / \alpha]$ problem (8.1) has a unique classical solution $u \in C^{\sigma}\left([0, T] ; \mathscr{D}\left((-A)^{\gamma}\right)\right)$. Moreover, the following estimate holds

$$
\|u\|_{\sigma, T ; \mathscr{D}\left((-A)^{\gamma}\right)} \leq c_{30}(T)\left\|u_{0}\right\|_{\mathscr{D}\left((-A)^{\varphi}\right)}+T^{(2 \alpha+\beta-\Re \mathrm{e} \gamma-2-\alpha \sigma) / \alpha} c_{29}(T)\|f\|_{0, T ; X} .
$$

Proof. First, since $2 \alpha+\beta>3-\beta \geq 2$, our choice for $\mu$ makes sense. Now, when $f \in C^{\mu}([0, T] ; X), \mu \in((2-\alpha-\beta) / \alpha, 1)$, and $u_{0} \in \mathscr{D}\left((-A)^{\varphi}\right)$, $\Re \mathrm{e} \varphi \in$ $(3-\alpha-2 \beta, \alpha) \subseteq(1-\beta, 1)$, from [12, Theorem 3.7 and Remark p. 54] it follows that problem (8.1) admits a unique classical solution. In particular, the following representation holds:

$$
u(t)=\mathrm{e}^{t A} u_{0}+\left[Q_{1} f\right](t), \quad t \in[0, T]
$$

$Q_{1}$ being defined by (7.8). Now, observe that $\Re$ e $\varphi<\alpha$ implies

$$
\begin{aligned}
& \Re \mathrm{e} \gamma \in(1-\beta, \alpha+\beta+\Re \mathrm{e} \varphi-2) \subsetneq(1-\beta, 2 \alpha+\beta-2) \\
& \sigma \in(0,(\alpha+\beta-\Re \mathrm{e} \gamma+\Re \mathrm{e} \varphi-2) / \alpha] \subsetneq(0,(2 \alpha+\beta-\Re \mathrm{e} \gamma-2) / \alpha),
\end{aligned}
$$

so that we are in position to apply both Lemmas 7.6 and 7.7. Hence $Q_{1} f$ and $\mathrm{e}^{\cdot A} u_{0}$ belong to $C^{\sigma}\left([0, T] ; \mathscr{D}\left((-A)^{\gamma}\right)\right)$ and the same belonging is true for $u$ by virtue of (8.3). Estimate (8.2) finally follows from (7.9), (7.10) and (8.3).

We now come to the main maximal regularity result of the section.

TheOREM 8.2. Let $3 \alpha+2 \beta>4$ and let $f \in C^{\mu}([0, T] ; X), \quad \mu \in((4-2 \alpha$ $-2 \beta) / \alpha, 1)$. Further, let $u_{0} \in \mathscr{D}(A)$ and assume there exists $u_{1} \in A u_{0}$ such that $u_{1}+f(0)=: g_{0} \in \mathscr{D}\left((-A)^{\varphi}\right), \quad \Re \mathrm{e} \varphi \in(3-\alpha-2 \beta, \alpha \mu+\alpha-1)$. Then, for every $\Re \mathrm{e} \gamma \in(1-\beta, \alpha+\beta+\Re \mathrm{e} \varphi-2)$ and $\sigma \in(0,(\alpha+\beta-\Re \mathrm{e} \gamma+\Re \mathrm{e} \varphi-2) / \alpha]$ problem 
(8.1) has a unique classical solution $u \in C^{1+\sigma}\left([0, T] ; \mathscr{D}\left((-A)^{\gamma}\right)\right)$. Moreover, the following estimate holds

$$
\begin{aligned}
\left\|D_{t} u\right\|_{\sigma, T ; \mathscr{D}\left((-A)^{\gamma}\right)} \leq & c_{30}(T)\left\|g_{0}\right\|_{\mathscr{D}\left((-A)^{\varphi}\right)} \\
& +T^{(\alpha \mu+2 \alpha+\beta-\Re \mathrm{e} \gamma-3-\alpha \sigma) / \alpha} c_{33}(T)|f|_{\mu, T ; X},
\end{aligned}
$$

where $c_{33}(T)=T^{(1-\alpha) / \alpha} c_{31}(T)+c_{32}(T)$.

Proof. Let us denote with $I_{\alpha, \beta}, I_{\alpha, \beta, \mu}, I_{\alpha, \beta, \varphi}$ and $I_{\alpha, \beta, \gamma, \varphi}$ the intervals $((4-2 \alpha-2 \beta) / \alpha, 1), \quad(3-\alpha-2 \beta, \alpha \mu+\alpha-1), \quad(1-\beta, \alpha+\beta+\Re \mathrm{e} \varphi-2) \quad$ and $(0,(\alpha+\beta-\Re \mathrm{e} \gamma+\Re \mathrm{e} \varphi-2) / \alpha]$, respectively. Then, since $\mu \in I_{\alpha, \beta} \subseteq((2-\alpha$ $-\beta) / \alpha, 1)$ and $u_{0} \in \mathscr{D}(A) \hookrightarrow \mathscr{D}\left((-A)^{\varphi}\right)$, $\Re \mathrm{e} \varphi \in I_{\alpha, \beta, \mu} \subsetneq(3-\alpha-2 \beta, \alpha)$, Theorem 8.1 applies and for every $\Re \mathrm{e} \gamma \in I_{\alpha, \beta, \varphi}$ and $\sigma \in I_{\alpha, \beta, \gamma, \varphi}$ problem (8.1) has a unique classical solution $u \in C^{\sigma}\left([0, T] ; \mathscr{D}\left((-A)^{\gamma}\right)\right)$ satisfying (8.2). Moreover, differentiating (8.3) with respect to $t$ and using $g_{0} \in \mathscr{D}\left((-A)^{\varphi}\right)$, it turns out that the derivative of the solution is given by (cf. [12, Remark p. 55])

$$
D_{t} u(t)=\mathrm{e}^{t A} g_{0}+\left[Q_{2} f\right](t)+\left[Q_{3} f\right](t), \quad t \in(0, T],
$$

$Q_{2}$ and $Q_{3}$ being defined by (7.15) and (7.17), respectively. Now, $\Re \mathrm{e} \varphi \in I_{\alpha, \beta, \mu}$ implies

$$
\begin{aligned}
& I_{\alpha, \beta, \varphi} \subsetneq(1-\beta, \alpha \mu+2 \alpha+\beta-3) \subseteq(1-\beta, \alpha \mu+\alpha+\beta-2), \\
& I_{\alpha, \beta, \gamma, \varphi} \subsetneq(0,(\alpha \mu+2 \alpha+\beta-\Re \mathrm{e} \gamma-3) / \alpha) \subseteq(0,(\alpha \mu+\alpha+\beta-\Re \mathrm{e} \gamma-2) / \alpha),
\end{aligned}
$$

and the assumptions in all of Lemmas 7.7, 7.10 and 7.11 are satisfied when $\Re \mathrm{e} \gamma \in I_{\alpha, \beta, \varphi}$ and $\sigma \in I_{\alpha, \beta, \gamma, \varphi}$. It then follows that for every $\Re \mathrm{e} \gamma \in I_{\alpha, \beta, \varphi}$ and $\sigma \in I_{\alpha, \beta, \gamma, \varphi}, \mathrm{e}^{A} g_{0}$ and $Q_{j} f, j=2,3$, belong to $C^{\sigma}\left([0, T] ; \mathscr{D}\left((-A)^{\gamma}\right)\right)$. Of course, due to $(8.5)$, the same belonging holds for $D_{t} u$. In particular, by setting $D_{t} u(0)=$ $g_{0} \in A u_{0}+f(0)$, we have $\lim _{t \rightarrow 0^{+}}\left\|D_{t} u(t)-D_{t} u(0)\right\|_{\mathscr{D}\left((-A)^{\gamma}\right)}=0$ and the equation in (8.1) is satisfied even at $t=0$. Finally, estimates (7.10), (7.16) and (7.18) yield (8.4), and the proof is complete.

Remark 8.3. Notice that $3 \alpha+2 \beta>4$ and $\beta \leq \alpha$ yield $\beta \in(2-(3 \alpha) / 2, \alpha$, so that $\alpha \in(4 / 5,1]$. In particular, if $\alpha=1$, then $\beta \in(1 / 2,1], \mu \in(2(1-\beta), 1)$, $\Re$ e $\varphi \in$ $(2(1-\beta), \mu), \Re \mathrm{e} \gamma \in(1-\beta, \beta+\Re \mathrm{e} \varphi-1)$ and $\sigma \in(0, \beta-\Re \mathrm{e} \gamma+\Re \mathrm{e} \varphi-1]$. Hence, in the optimal case $(\alpha, \beta)=(1,1)$, we have $\mu \in(0,1), \Re \mathrm{e} \varphi \in(0, \mu), \Re \mathrm{e} \gamma \in(0, \Re \mathrm{e} \varphi)$, $\sigma \in(0,-\Re \mathrm{e} \gamma+\Re \mathrm{e} \varphi]$. 
REMARK 8.4. The peculiar feature of both Theorems 8.1 and 8.2 is that they exhibit a sort prevalence for the space regularity. We mean, the larger is the real part of the parameter $\gamma$ in the domain $\mathscr{D}\left((-A)^{\gamma}\right)$ where we look for the space regularity, the smaller is the corresponding interval where the Hölder exponent $\sigma$ of regularity in time may vary.

Theorem 8.2 enable us to treat questions of maximal regularity for the problem

$$
D_{t}(M v(t))=L v(t)+f(t), \quad t \in(0, T], \quad M v(0)=u_{0},
$$

where $M$ and $L$ are two single-valued closed linear operators from $X$ to itself whose domains satisfy $\mathscr{D}(L) \subseteq \mathscr{D}(M)$. We allow $M$ to have no bounded inverse, so that, in general, $M^{-1}$ is the m. 1 . operator in $X$ defined by $M^{-1} y=$ $\{x \in \mathscr{D}(M): y=M x\}, y \in \mathscr{D}\left(M^{-1}\right)=\mathscr{R}(M)$. As a consequence, problem (8.6) may have a degeneration in the time derivative and for this reason is called degenerate. Clearly, if $M=I$, then problem (8.6) is non-degenerate and for this particular case the question of maximal regularity is nowadays well-known (see [27] and the references therein).

We recall that the $M$-modified resolvent set $\rho_{M}(L)$ of $L$ is the set of all $z \in \mathbf{C}$ such that $\mathscr{R}(z M-L)=X$ and $(z M-L)^{-1} \in \mathscr{L}(X)$. It is shown in [12, Theorem 1.14] that

$$
\rho_{M}(L) \subseteq \rho\left(L M^{-1}\right) \quad \text { and } \quad M(z M-L)^{-1}=\left(z I-L M^{-1}\right)^{-1}, \quad z \in \rho_{M}(L) .
$$

With the notion of $M$-modified resolvent set of $L$ at hand, we assume:

(H2) $\rho_{M}(L)$ contains a region $\Sigma_{\alpha}=\left\{z \in \mathbf{C}: \Re \mathrm{e} z \geq-c(|\Im \mathrm{m} z|+1)^{\alpha}\right.$, গm $z \in \mathbf{R}\}, \alpha \in(0,1], c>0$, and for some $\beta \in(0, \alpha]$ and $C>0$ it holds that:

$$
\left\|M(\lambda M-L)^{-1}\right\|_{\mathscr{L}(X)} \leq C(|\lambda|+1)^{-\beta}, \quad \forall \lambda \in \Sigma_{\alpha} .
$$

Let $A$ be the (possibly) m. 1. operator $L M^{-1}$. Then (cf. (2.1) with $\left(A_{1}, A_{2}\right)=$ $\left.\left(L, M^{-1}\right)\right)$

$$
\left\{\begin{aligned}
\mathscr{D}(A) & =\left\{u \in \mathscr{D}\left(M^{-1}\right): \mathscr{D}(L) \cap M^{-1} u \neq \varnothing\right\} \\
& =\left\{u \in \mathscr{R}(M): \text { there exists } v \in \mathscr{D}(L) \text { such that } v \in M^{-1} u\right\} \\
& =\{u \in \mathscr{R}(M): u=M v \text { for some } v \in \mathscr{D}(L)\}=M(\mathscr{D}(L)), \\
A u= & \bigcup_{v \in \mathscr{D}(L) \cap M^{-1} u} L v=\{L v: v \in \mathscr{D}(L) \text { such that } u=M v\}, \quad u \in \mathscr{D}(A) .
\end{aligned}\right.
$$

Of course, due to (8.7), assumption (H2) implies that $A$ fulfills assumption (H1) and hence that it generates a semigroup $\left\{\mathrm{e}^{t A}\right\}_{t \geq 0}$ defined by (2.10) and satisfying (2.12), (3.20), (3.22) and (3.23). The m. 1. operator $A$ naturally appears when we 
rewrite (8.6) into an equivalent non-degenerate form. In fact, changing the unknown function to $u=M v$, problem (8.6) turns out to be completely equivalent to the multivalued evolution problem (8.1) with $A$ defined by (8.8). By virtue of this equivalence between, we then say that a function $v \in C((0, T] ; \mathscr{D}(L))$ is a strict (respectively, classical) solution to (8.6) if $u=M v$ is a strict (respectively, classical) solution to (8.1) with $A=L M^{-1}$.

To our knowledge, under assumption $(\mathrm{H} 2)$, the first results of time regularity for the strict solutions to (8.6) are [10, Theorem 9] and [12, Theorem 3.26]. In particular, the quoted results show that, unless $\beta=1$, the time regularity of $D_{t} M v$ decreases with respect to that of $f$. Precisely, if $f \in C^{\mu}([0, T] ; X), \mu \in((2-\alpha-\beta) /$ $\alpha, 1), 2 \alpha+\beta>2$, and $u_{0}=M v_{0}$, where $v_{0} \in \mathscr{D}(L)$ satisfies $L v_{0}+f(0) \in X_{A}^{\gamma, \infty}$, $\gamma=\alpha^{2} \mu+(2-\alpha-\beta)(1-\alpha)$, then $D_{t} M v \in C^{v}([0, T] ; X), v=\alpha \mu+\alpha+\beta-2$.

Concerning space regularity, instead, the first result is that given in [10, Theorem 5], but only for $\beta=1$ in (H2). In this case $\mathscr{T}=\left.A^{-1}\right|_{\mathscr{R}\left(A^{-1}\right)}=$ $\left.\left(M L^{-1}\right)\right|_{\mathscr{D}(A)}$ has an inverse $\mathscr{T}^{-1}$ which is the generator of an analytic semigroup in $\overline{\mathscr{D}(A)}$. Let $P$ the projection operator onto $\mathscr{N}\left(A^{-1}\right)=A 0$ and assume $f \in C([0, T] ; X)$ and $(I-P) L v_{0} \in X_{\mathscr{T}^{-1}}^{\gamma, \infty}$, where $\gamma \in(0,1)$ and $u_{0}=M v_{0}, v_{0} \in$ $\mathscr{D}(L)$. Then in [10, Theorem 5] it is shown that $D_{t} M v$ is bounded from $[0, T]$ to $X_{\mathscr{T}^{-1}}^{\gamma, \infty}$ provided that $(I-P) f$ is.

Recently, the results in [10] and [12] have been improved in [8, Theorem 5.3]. There, under assumption (H2) and without invoking any projection operator, an optimal "cross" regularity results is established, in which regularity in both time and space is provided for $M v$ and $D_{t} M v$. Precisely, let $\beta \in(1-\alpha / 2,2 \alpha-1]$, $\alpha \in(4 / 5,1]$, and let $f \in C^{\mu}([0, T] ; X), \mu \in((3-2 \alpha-\beta) / \alpha,(1+\beta-\alpha) / \alpha)$. Assume $u_{0}=M v_{0} \in \mathscr{D}(A)$, where $v_{0} \in \mathscr{D}(L)$ is such that $L v_{0}+f(0) \in X_{A}^{\varphi, 1}, \varphi \in(2-\alpha-\beta$, $\alpha \mu+\alpha-1)$. Then, for every $\gamma \in(0, \alpha+\beta+\varphi-2), \sigma \in(0,(\alpha+\beta-\gamma+\varphi-2) / \alpha]$ and $p \in[1, \infty]$, problem (8.6) has a unique classical solution $v$ such that $M v \in$ $C^{1+\sigma}\left([0, T] ;(X, \mathscr{D}(A))_{\gamma, p}\right)$.

Since $X_{A}^{\varphi, 1} \subseteq\{0\} \cup\left[\mathscr{D}\left((-A)^{\varphi}\right) \backslash A 0\right]$ for $\varphi \in(1-\beta, \beta)$, [8, Theorem 5.3] is not applicable if $L v_{0}+f(0) \in \mathscr{D}\left((-A)^{\varphi}\right) \backslash X_{A}^{\varphi, 1}$. It is in this case that Theorem 8.2 turns out to be particularly useful, allowing us to prove time and space regularity for the solutions to (8.6) even when the data are less smooth than what is required in [8]. The only main difference is that now the space regularity is established with respect to the domains $\mathscr{D}\left((-A)^{\gamma}\right)$ and no more with respect to the interpolation spaces $(X, \mathscr{D}(A))_{\gamma, p}$.

THeOREm 8.5. Let $(\mathrm{H} 2)$ be satisfied with $3 \alpha+2 \beta>4$ and let $f \in C^{\mu}([0, T] ; X)$, $\mu \in((4-2 \alpha-2 \beta) / \alpha, 1)$. Further, $A$ being the $m$. l. operator $L M^{-1}$ defined by 
(8.8), let $u_{0}=M v_{0} \in \mathscr{D}(A)$ where $v_{0} \in \mathscr{D}(L)$ is such that $L v_{0}+f(0)=: g_{0} \in$ $\mathscr{D}\left((-A)^{\varphi}\right)$, $\Re \mathrm{e} \varphi \in(3-\alpha-2 \beta, \alpha \mu+\alpha-1)$. Then, for every $\Re \mathrm{e} \gamma \in(1-\beta, \alpha+\beta+$ $\Re \mathrm{e} \varphi-2)$ and $\sigma \in(0,(\alpha+\beta-\Re \mathrm{e} \gamma+\Re \mathrm{e} \varphi-2) / \alpha]$ problem (8.6) has a unique classical solution $v$ such that $u=M v \in C^{1+\sigma}\left([0, T] ; \mathscr{D}\left((-A)^{\gamma}\right)\right)$. Moreover, estimate (8.4) holds for $D_{t} u=D_{t} M v$.

Proof. It suffices to rewrite problem (8.6) into the equivalent nondegenerate multivalued form (8.1) with $(u, A)=\left(M v, L M^{-1}\right)$ and to apply Theorem 8.2 with $u_{1}=L v_{0} \in A u_{0}$.

We now apply Theorem 8.5 to a concrete case deeply investigated in [10][12]. Let $\Omega \subset \mathbf{R}^{n}$ be a bounded region with a smooth boundary $\partial \Omega$ and consider the problem:

$$
\left\{\begin{array}{l}
D_{t}(m(x) v(t, x))=L\left(x ; D_{x}\right) v(t, x)+f(t, x), \quad(t, x) \in(0, T] \times \Omega, \\
v(t, x)=0, \quad(t, x) \in(0, T] \times \partial \Omega, \quad m(x) v(0, x)=u_{0}(x), \quad x \in \Omega .
\end{array}\right.
$$

Here $m(x) \geq 0, f(t, x)$ and $u_{0}(x)$ are three given functions whose regularity will be specified later, while $L\left(x ; D_{x}\right)=\sum_{i, j=1}^{n} D_{x_{i}}\left(a_{i, j}(x) D_{x_{j}}\right)-a_{0}(x)$. The coefficients $a_{i, j}$ and $a_{0}$ of $L\left(x ; D_{x}\right)$ are assumed to satisfy the following properties:

$$
\begin{gathered}
a_{i, j}=a_{j, i} \in C^{1}(\overline{\mathbf{\Omega}}), \quad i, j=1, \ldots, n, \quad a_{0} \in C(\overline{\mathbf{\Omega}}), \quad a_{0}(x) \geq v_{1}, \quad \forall x \in \overline{\mathbf{\Omega}} \\
v_{2}|\xi|^{2} \leq \sum_{i, j=1}^{n} a_{i, j}(x) \xi_{i} \xi_{j} \leq v_{3}|\xi|^{2}, \quad \forall(x, \xi) \in \overline{\mathbf{\Omega}} \times \mathbf{R}^{n}
\end{gathered}
$$

$v_{k}, k=1,2,3$, being three positive constants. Of course, problem (8.9) can be reformulated in an abstract way as a problem of type (8.6), in which $M$ is the multiplication operator by the function $m$ and $L$ is $L\left(x ; D_{x}\right)$ with Dirichlet boundary conditions. To this purpose, we take $X=L_{q}(\Omega), q \in[1, \infty]$, endowed with the standard norm $\|\cdot\|_{q}$. The operator $L$ is then defined by

$$
\mathscr{D}(L)=W_{q}^{2}(\Omega) \cap \stackrel{\circ}{W}_{q}^{1}(\Omega), \quad L u=L\left(x ; D_{x}\right) u, \quad u \in \mathscr{D}(L) .
$$

Here (cf. [17, Chapter 7]), $W_{q}^{k}(\Omega), k \in \mathbf{N} \cup\{0\}, q \in[1, \infty]$, is the usual Sobolev space endowed with the norm $\|\cdot\|_{k, q}\left(\left(W_{q}^{0}(\Omega),\|\cdot\|_{0, q}\right)=\left(L^{q}(\Omega),\|\cdot\|_{q}\right)\right)$, whereas $\stackrel{\circ}{W}_{q}^{k}(\Omega)$ denotes the completion of $C_{0}^{\infty}(\Omega)$ in $W_{q}^{k}(\Omega), C_{0}^{\infty}(\Omega)$ being the set of all complex-valued infinitely differentiable functions defined over $\Omega$ with compact support. Assume now $m \in L_{\infty}(\Omega)$, so that $M \in \mathscr{L}(X), M v=m v, v \in X$. Hence the assumption $\mathscr{D}(L) \subseteq \mathscr{D}(M)$ is satisfied and the m. 1. operator $A=L M^{-1}$ is determined by $(8.8)$ with $\mathscr{D}(L)$ as in $(8.10)$. 
By referring to [8] and the references therein for the proof, we now recall that for every fixed $q \in(1, \infty)$, the following estimates holds (cf. [8, formula (77)]):

$$
\left\|M(\lambda M-L)^{-1}\right\|_{\mathscr{L}(X)} \leq C(|\lambda|+1)^{-1 / q}, \quad \forall \lambda \in \Sigma_{1},
$$

where $\Sigma_{1}=\{z \in \mathbf{C}: \Re \mathrm{e} z>-c(|\Im \mathrm{m} z|+1), \Im \mathrm{m} z \in \mathbf{R}\}, c$ being a suitable positive constant depending on $q$ and $\|m\|_{L^{\infty}(\Omega)}$. Therefore, $(\mathrm{H} 2)$ is satisfied with $(\alpha, \beta)=$ $(1,1 / q)$ and the condition $\beta \in(1 / 2,1]$ necessary to apply Theorem 8.5 with $\alpha=1$ yields $q \in(1,2)$. So, let $q \in(1,2)$ and denote with $q^{\prime}$ the conjugate exponent of $q$. Recalling Remark 8.3 assume that $f \in C^{\mu}([0, T] ; X), \mu \in\left(2 / q^{\prime}, 1\right)$, and that $u_{0}=m v_{0} \in \mathscr{D}(A)$, where $v_{0} \in \mathscr{D}(L)$ is such that $L v_{0}+f(0, \cdot)=g_{0} \in \mathscr{D}\left((-A)^{\varphi}\right)$, $\Re \mathrm{e} \varphi \in\left(2 / q^{\prime}, \mu\right)$. Then, from Theorem 8.5 with $(\alpha, \beta)=(1,1 / q)$ we deduce that for every $\Re$ e $\gamma \in\left(1 / q^{\prime}, \Re \mathrm{e} \varphi-1 / q^{\prime}\right), \sigma \in\left(0,-\Re \mathrm{e} \gamma+\Re \mathrm{e} \varphi-1 / q^{\prime}\right]$ problem (8.9) has a unique classical solution $v$ such that $m v \in C^{1+\sigma}\left([0, T] ; \mathscr{D}\left((-A)^{\gamma}\right)\right)$. In addition the following estimate holds

$$
\left\|D_{t}(m v)\right\|_{\sigma, T ; \mathscr{D}\left((-A)^{\gamma}\right)} \leq c_{30}(T)\left\|g_{0}\right\|_{\mathscr{D}\left((-A)^{\varphi}\right)}+T^{\mu-\Re \mathrm{e} \gamma-\sigma-1 / q^{\prime}} c_{33}(T)|f|_{\mu, T ; X} .
$$

Values of $q$ larger than two can be obtained assuming more smoothness and some order of vanishing for the function $m$ on $\overline{\boldsymbol{\Omega}}$. Indeed, let $m \in C^{1}(\overline{\mathbf{\Omega}})$ be such that the following estimate holds for some $\varrho \in(0,1)$ and some positive constant $K$ :

$$
|\nabla m(x)|:=\left\{\sum_{j=1}^{n}\left[D_{x_{j}} m(x)\right]^{2}\right\}^{1 / 2} \leq K[m(x)]^{\varrho}, \quad x \in \overline{\mathbf{\Omega}} .
$$

Then (8.11) holds with $\beta=1 / q$ being replaced by (cf. [8, formula (80)]):

$$
\beta=(2-\varrho)^{-1}, \quad \text { if } q \in(2-\varrho, 2), \quad \beta=2[q(2-\varrho)]^{-1}, \quad \text { if } q \in[2, \infty) .
$$

Then, in order that the condition $\beta \in(1 / 2,1]$ in Theorem 8.5 with $\alpha=1$ is satisfied, it suffices to take $q \in(2-\varrho, 2) \cup[2,4 /(2-\varrho))$.

As another application we consider the following degenerate equation in $X=L^{2}(\Omega)$, where $\Omega \subset \mathbf{R}^{n}$ is as before:

$$
\left\{\begin{array}{l}
D_{t}(u(t, x))=\Delta_{x}\{a(x) u(t, x)\}+f(t, x), \quad(t, x) \in(0, T] \times \Omega, \\
a(x) u(t, x)=0, \quad(t, x) \in(0, T] \times \partial \Omega, \quad u(0, x)=u_{0}(x), \quad x \in \Omega .
\end{array}\right.
$$

Here, $\Delta_{x}$ denotes the usual Laplace partial differential operator in $\mathbf{R}^{n}$, whereas $a \in L^{\infty}(\Omega)$ is a nonnegative given function such that $a(x)>0$ almost everywhere in $\Omega$. The change of unknown $v(t, x)=a(x) u(t, x)$ yields to rewrite problem (8.12) 
in the form (8.6), in which $M$ is the multiplication operator by the function $m(x)=1 / a(x)$ and $L: W_{2}^{2}(\Omega) \cap \stackrel{\circ}{W} \underset{2}{1}(\Omega) \rightarrow L^{2}(\Omega)$ is $\Delta_{x}$ with the Dirichlet boundary condition. Let assume that

$$
m=\frac{1}{a} \in\left\{\begin{array}{l}
L^{r}(\Omega) \text { with some } r \geq 2 \text { when } n=1, \\
L^{r}(\Omega) \text { with some } r>2 \text { when } n=2, \\
L^{r}(\Omega) \text { with some } r \geq n \text { when } n \geq 3 .
\end{array}\right.
$$

Then (cf. [8, p. 270]), the following estimate holds

$$
\left\|M(\lambda M-L)^{-1}\right\|_{\mathscr{L}(X)} \leq C(|\lambda|+1)^{-[1-n /(2 r)]}, \quad \forall \lambda \in \Sigma_{1},
$$

so that (H2) is satisfied with $(\alpha, \beta)=(1,1-n /(2 r))$. Hence $\beta \in(1 / 2,1]$ implies $r>n$ if $n \geq 3$. Let $A$ be the m. 1. operator defined by (8.8) with $\mathscr{D}(L)=$ $W_{2}^{2}(\Omega) \cap \stackrel{\circ}{W}_{2}^{1}(\Omega)$ and let $f \in C^{\mu}([0, T] ; X), \mu \in(n / r, 1)$. Assume $u_{0}=m v_{0} \in \mathscr{D}(A)$ where $v_{0} \in \mathscr{D}(L)$ is such that $L v_{0}+f(0, \cdot)=g_{0} \in \mathscr{D}\left((-A)^{\varphi}\right)$, $\Re \mathrm{e} \varphi \in(n / r, \mu)$. From Theorem 8.5 it thus follows that for every $\Re \mathrm{e} \gamma \in(n /(2 r), \Re \mathrm{e} \varphi-n /(2 r))$ and $\sigma \in(0,-\Re \mathrm{e} \gamma+\Re \mathrm{e} \varphi-\gamma-n /(2 r)]$ problem (8.12) has a unique classical solution $u=m v \in C^{1+\sigma}\left([0, T] ; \mathscr{D}\left((-A)^{\gamma}\right)\right)$. Further, the time derivative $D_{t} u=D_{t}(m v)$ satisfies estimate (8.4) with $v=\mu-\Re \mathrm{e} \gamma-\sigma-n /(2 r)$.

We now suggest a possible application of our results to abstract multivalued semilinear initial value problems of the kind

$$
D_{t} u(t) \in A u(t)+f(t, u(t)), \quad t \in(0, T], \quad u(0)=u_{0},
$$

$A$ being a m. 1. operator satisfying (H1). It is well-known (cf. [20, Chapter 3]) that if $A$ is single-valued and satisfies (H1) with $\beta=1$, then problem (8.13) can be solved assuming that $f \in C([0, T] \times \mathcal{O} ; X)$ is locally Hölder continuous in $t \in[0, T]$ and locally Lipschitz in $x \in \mathcal{O}, \mathcal{O}$ being an open subset of $\mathscr{D}\left((-A)^{\gamma}\right)$, $\gamma \in(0,1)$. Under these assumptions on $f$ it is shown in [20, Theorem 3.3.3] that for every $u_{0} \in \mathcal{O}$ there exists $T_{1}=T_{1}\left(u_{0}\right) \in(0, T]$ such that problem (8.13) has a unique solution $u$ in $\left(0, T_{1}\right)$ with initial value $u(0)=u_{0}$. The global existence is then shown in [20, Theorem 3.3.5] under the additional requirement that there exist a continuous function $\Theta:[0, \infty) \rightarrow[0, \infty)$ such that $\|f(t, x)\|_{X} \leq$ $\Theta(t)\left(1+\|x\|_{X_{\gamma}}\right)$ for every $(t, x) \in[0, T] \times \mathcal{O}$. We refer also to [21], where the local and global existence for the single-valued version of problem (8.13) is treated under the weak assumption $u_{0} \in \mathscr{D}\left((-A)^{\delta}\right), \delta \in[0, \gamma]$, but assuming $f$ independent of time. The case when $A$ is single-valued, $\alpha=1$ and $\beta \in(0,1)$ has been recently considered in [29] under the assumptions that $f \in C([0, T] \times X ; X)$ is locally Lipschitz in $x \in X$ and that $u_{0} \in \mathscr{D}\left((-A)^{\theta}\right), \theta \in(1-\beta, \beta)$. It seems to us that 
embedding (6.36) with $A 0=\{0\}$ may be used to generalize the results in [29] to the case $\alpha<1$. Of course, in this case the semigroup generated by $A$ is no longer analytic and some details in the proofs of the main results in [29] should be opportunely modified.

Contrarily to the single-valued case, when $A$ is really a m. 1. operator a general solvability theory for problem (8.13) is still lacking. Maybe, embedding (6.24) and/or (6.36) could be a first step in this direction. Recall that the linear subspace $A 0$ is closed on $X$ and hence $\mathscr{D}\left((-A)^{\theta}\right) \backslash A 0$ turns out to be an open subset of $\mathscr{D}\left((-A)^{\theta}\right)$. This suggests that the assumption $f \in C([0, T] \times \mathcal{O} ; X), \mathcal{O}$ being an open subset of $\mathscr{D}\left((-A)^{\gamma}\right), \gamma \in(0,1)$, used to treat the single-valued case should be replaced by $f \in C([0, T] \times(\{0\} \cup \mathcal{O}) ; X)$, where $\mathcal{O}=\mathscr{D}\left((-A)^{\gamma}\right) \backslash A 0$ is the open subset of $\mathscr{D}\left((-A)^{\gamma}\right)$ having the desired intermediate property for $\gamma \in(2-\alpha-\beta, \beta)$. Even if this were the case, another difficulty is that the usual procedure for uniquely solving problem (8.13) through a contraction argument does not work when $A$ is really a m. 1 . operator. Indeed, in this case we have to employ a fixed-point theorem for $\mathrm{m}$. 1. operators, and fixed-point theorems for m. 1. operators generally provide existence but not uniqueness.

We conclude the section spending some words on the possible applications of our results to multivalued non autonomous linear initial value problems of the type

$$
D_{t} w(t) \in A(t) w(t)+f(t), \quad t \in(0, T], \quad w(0)=u_{0},
$$

where $A(t)$ satisfies condition $(\mathrm{H} 1)_{t}$ for every $t \in[0, T],(\mathrm{H} 1)_{t}$ being $(\mathrm{H} 1)$ with the pair $(\rho(A), A)$ replaced by $(\rho(A(t)), A(t))$. The case when $A(t)$ is single-valued for every $t \in[0, T]$ and $\beta=1$ in $(\mathrm{H} 1)_{t}$ has been studied by many authors. See, for instance, the papers [2], [3], [33] and [39], and the books [27, Chapter 6] and [34, Chapter 6]. Essentially, there are two different approach for solving problem (8.14) in the single-valued case with $\beta=1$, according that the operators $A(t)$ have or not constant domains. The case of constant domains $\mathscr{D}(A(t)) \equiv \mathscr{D}$ is considered in [27], [33] and [34] where, under the additional assumption that the map $t \rightarrow A(t)$ belongs to $C^{\sigma}([0, T] ; \mathscr{L}(\mathscr{D} ; X))$ for some $\sigma \in(0,1)$, it is shown that there exists an evolution operator for problem (8.14). In the case of variable domains several types of assumptions can be made to solve problem (8.14), but they can be roughly divided in two groups. The first group of assumptions concerns the case where the map $t \rightarrow(z I-A(t))^{-1}$ is very regular for every $z \in \rho(A(t))$ (cf. [39]). The second group refers instead to the case where, although the dense domains $\mathscr{D}(A(t))$ may vary, one between the intermediate spaces $\mathscr{D}\left((-A(t))^{\gamma}\right)$ and $(X, \mathscr{D}(A(t)))_{\gamma, \infty}$ is constant for some $\gamma \in(0,1)$ (cf. [2] and [3]). As far as the 
single-valued case with $\alpha=1$ and $\beta \in(0,1)$ in $(\mathrm{H} 1)_{t}$ it is concerned, this has been treated in [36] and [37], but only in the concrete situation $X=C^{\gamma}(\overline{\mathbf{\Omega}})$, where $\gamma \in(0,1)$ and $\Omega \subset \mathbf{R}^{n}$ is a bounded domain having smooth boundary. In that papers the operators $A(t)$ are elliptic operators of order $2 l, l \in \mathbf{N}$, having constant domains $C^{2 l+\gamma}(\overline{\mathbf{\Omega}})$, and the estimate $\left\|(\lambda I-A(t))^{-1}\right\|_{\mathscr{L}(X)} \leq C(1+|\lambda|)^{-\beta}, \lambda \in \Sigma_{1}$, it is shown to hold with $\beta=1-\gamma /(2 l)$.

Since the intermediate property of the domains of the fractional powers of $-A(t)$ plays a key role in the approach followed in [2], it is our opinion that embedding (6.36) can be used to extend the results in that paper to single-valued linear operators $A(t)$ which satisfies $(\mathrm{H} 1)_{t}$ with $(\alpha, \beta) \neq(1,1)$ and whose domains are neither constant nor dense in $X$. At moment, the main difficult in carrying out this program is to find how to modify the proofs of [2], in order to avoid there the use of identity (4.27).

If the operators $A(t)$ are really $\mathrm{m}$. 1. operators which satisfies $(\mathrm{H} 1)_{t}$ and whose not necessarily dense domains are allowed to vary, then problem (8.14) can be solved provided that three additional types of assumptions are made on the operators $A(t)$ and on the exponent pair $(\alpha, \beta)$ (cf. assumptions (A.j), (Ex.j), $\mathrm{j}=\mathrm{i}, \mathrm{ii}$, iii, in [12, Chapter 4]). Also, the first of these assumptions is precisely the multivalued version of that used in [39] to solve problem (8.14) in the singlevalued case with $\beta=1$. Hence, in a multivalued sense, the assumptions in [12] belong to the first of the two groups mentioned before. It is an open question if embedding (6.36) can be used to solve (8.14), adapting to the multivalued case the approach followed in [2] in the single-valued case with $\beta=1$. In other words, we query if, due to (6.36), the second group of assumptions used to solve (8.14) in the single-valued case with $\beta=1$, can be used even for solving (8.14) in the multivalued case with $(\alpha, \beta) \neq(1,1)$.

\section{The m. I. Operators $(-A)^{ \pm \theta}$ for $\Re \mathrm{e} \theta \in[0,1-\beta]$}

We provide here a possible definition for the fractional powers $(-A)^{ \pm \theta}$, $\Re$ e $\theta \in[0,1-\beta]$, of a m. 1 . operator $A$ satisfying assumption (H1). Our approach will furnish not only the positive fractional powers $(-A)^{\theta}, \Re$ e $\theta \in(0,1-\beta]$, but also the non positive fractional powers $(-A)^{-\theta}, \theta \neq 0, \Re \mathrm{e} \theta \in[0,1-\beta]$, as a class of $\mathrm{m}$. 1. operators.

In order to motivate our next assumption (H3), we still recall the statement of [24, Proposition 2.9]: let $A$ be a single-valued densely defined linear operator satisfying (H1) with $\beta=1$. If there exists $\zeta_{1} \in \mathbf{C}$ such that $\Re$ e $\zeta_{1} \in(0,1)$ and $\mathscr{D}\left((-A)^{\zeta_{1}}\right)$ coincides, in the sense of equivalence of the norms, with $X_{A}^{\Re e} \zeta_{1}, p$ for 
some $p \in[1, \infty]$, then $\mathscr{D}\left((-A)^{\zeta_{2}}\right)=X_{A}^{\Re \mathrm{e} \zeta_{2}, p}$ for every $\zeta_{2} \in \mathbf{C}$ such that $\Re \mathrm{e} \zeta_{2} \in$ $(0,1)$. In particular, $\mathscr{D}\left((-A)^{\zeta_{1}}\right)=\mathscr{D}\left((-A)^{\zeta_{2}}\right)$ if $\Re \mathrm{e} \zeta_{1}=\Re \mathrm{e} \zeta_{2}$. In [24] this assertion is proved not only for $\Re \mathrm{e} \zeta_{k} \in(0,1)$, but for all $\Re \mathrm{e} \zeta_{k}>0, k=1,2$, provided to replace $X_{A}^{\Re \mathrm{e} \zeta_{k}, p}$ with $\mathscr{D}_{p}^{\Re \mathrm{e} \zeta_{k}}$, where the spaces $\mathscr{D}_{p}^{\gamma}, \gamma>0, p \in[1, \infty]$, are defined in [24, Definition 1.1] and coincide with $X_{A}^{\gamma, p}$ for $\gamma \in(0,1)$.

Let now $A$ be a m. 1. operator satisfying (H1) with $\alpha \in(1 / 2,1]$ and $\beta \in(1 / 2, \alpha]$. We make the following further assumption on $A$ :

(H3) for every $\zeta \in \mathbf{C}$ such that $\Re \mathrm{e} \zeta \in[\beta, 1]$ it holds that $\mathscr{R}\left((-A)^{-\zeta}\right)=\mathscr{D}(A)$.

Notice that $(\mathrm{H} 3)$ is meaningful, since $\beta \in(1 / 2, \alpha]$ implies $1-\beta<\beta$ and the fractional powers $(-A)^{-\zeta}$ are well defined for $\Re \mathrm{e} \zeta \in[\beta, 1]$. From (H3) we get $\mathscr{D}\left((-A)^{\zeta}\right)=\mathscr{D}(A)$ for every $\Re \mathrm{e} \zeta \in[\beta, 1]$. Hence, if $\zeta_{1}, \zeta_{2} \in \mathbf{C}$ are such that $\Re \mathrm{e} \zeta_{1}=\Re \mathrm{e} \zeta_{2} \in[\beta, 1]$, then $\mathscr{D}\left((-A)^{\zeta_{1}}\right)=\mathscr{D}\left((-A)^{\zeta_{2}}\right)$. In this sense, (H3) is inspired by [24, Proposition 2.9].

With assumption (H3) at hand, we can now proceed in our definition of the fractional powers $(-A)^{ \pm \theta}, \Re \mathrm{e} \theta \in[0,1-\beta]$ as follows. We set

$$
\begin{aligned}
& (-A)^{-\theta}:=\left\{\begin{array}{l}
I, \quad \text { if } \theta=0, \\
(-A)^{1-\theta}(-A)^{-1}, \quad \text { if } \theta \neq 0, \Re \mathrm{e} \theta \in[0,1-\beta],
\end{array}\right. \\
& (-A)^{\theta}:=\left((-A)^{-\theta}\right)^{-1}, \quad \Re \mathrm{e} \theta \in(0,1-\beta], \quad(-A)^{-\theta} \text { defined by }(9.1) .
\end{aligned}
$$

Definition (9.1) is meaningful, for the m. 1. operators $(-A)^{1-\theta}$ and $(-A)^{1-\theta}(-A)^{-1}$ on the right-hand side are both well defined. Indeed, since when $\Re$ e $\theta \in[0,1-\beta]$ we have $\Re \mathrm{e}(1-\theta) \in[\beta, 1]$, the fractional power $(-A)^{1-\theta}$ is defined as usual through (3.6) as the inverse of $(-A)^{-(1-\theta)}$. Moreover, by virtue of (H3), it has domain $\mathscr{D}\left((-A)^{1-\theta}\right)=\mathscr{R}\left((-A)^{-(1-\theta)}\right)=\mathscr{D}(A)$, and hence the composition $(-A)^{1-\theta}(-A)^{-1}$ is well defined, too. According to the second in (2.1) and since $A^{-1} \in \mathscr{L}(X)$, from (9.1) and (9.2) we get

$$
\left\{\begin{array}{l}
(-A)^{-\theta} x=\left\{(-A)^{1-\theta} y: y=(-A)^{-1} x\right\}, \quad x \in X, \theta \neq 0, \Re \mathrm{e} \theta \in[0,1-\beta], \\
(-A)^{\theta} x=\left\{y \in X: x \in(-A)^{-\theta} y\right\}, \quad x \in \mathscr{R}\left((-A)^{-\theta}\right), \Re \mathrm{e} \theta \in(0,1-\beta] .
\end{array}\right.
$$

Therefore, the operators $(-A)^{ \pm \theta}, \theta \neq 0, \Re \mathrm{e} \theta \in[0,1-\beta]$, are a family of $\mathrm{m} .1$. operators. In particular, from (9.1) and the first of (9.3) with $\theta=-i t$, we obtain that the purely imaginary powers $(-A)^{i t}$ of $-A$ are the $\mathrm{m}$. 1 . operators defined as follows

$$
\left\{\begin{array}{l}
(-A)^{i t}=(-A)^{1+i t}(-A)^{-1}, \quad \mathscr{D}\left((-A)^{i t}\right)=X, \quad \forall t \in \mathbf{R} \backslash\{0\} \\
(-A)^{i t} x=\left\{(-A)^{1+i t} y: y=(-A)^{-1} x\right\}, \quad x \in X, t \in \mathbf{R} \backslash\{0\}
\end{array}\right.
$$


REMARK 9.1. Let $A$ be a single-valued linear operator having dense domain and satisfying (H1) with $\beta=1$. In this case (9.4) is precisely the formula used in [24, p. 97] to show that, if the assumption of [24, Proposition 2.9] is satisfied, then $(-A)^{i t} \in \mathscr{L}(X)$ for every $t \in \mathbf{R}$. In fact, in this case the relation $\mathscr{D}\left((-A)^{1+i t}\right)=$ $\mathscr{D}(A)$ follows from the thesis of [24, Proposition 2.9] extended to all $\Re \mathrm{e} \zeta>0$ as explained before, whereas the identity $(-A)^{i t}=(-A)^{1+i t}(-A)^{-1}$ follows from [23, Corollary 7.4]. Of course, here $(-A)^{k+i t}=(-A)_{0}^{k+i t}, k=0,1$, where $(-A)_{0}^{\zeta}$, $\zeta \in \mathbf{C}$, is defined in Remark 3.7. Therefore, $(-A)^{i t}, t \in \mathbf{R}$, turns out to be a closed single-valued linear operator having domain the whole $X$, and hence bounded from the closed graph theorem. Clearly, as observed in $[35, \mathrm{p} .103]$, the difficult part in this proof of the boundedness of $(-A)^{i t}$ is to verify that the assumption $\mathscr{D}\left((-A)^{\zeta}\right)=\mathscr{D}_{p}^{\Re e}$ of [24, Proposition 2.9] is satisfied for some $\Re$ e $\zeta>0$ and some $p \in[1, \infty]$. Due to this difficulty, the local boundedness of $(-A)^{i t}$ it is a priori assumed in the proof of (4.27) in [35, Theorem 1.15.3].

REMARK 9.2. Observe that without assumption (H3) the composition $(-A)^{1-\theta}(-A)^{-1}$ in the second of (9.1) may become meaningless, since it is not guaranteed that $(-A)^{1-\theta}, \theta \neq 0, \Re \mathrm{e} \theta \in[0,1-\beta]$, is defined on the range of $(-A)^{-1}$, that is on $\mathscr{D}(A)$. Indeed, from $(3.8)$ with $\left(\theta_{1}, \theta_{2}\right)=(1,1-\theta)$ we can only ensure that the inclusion $\mathscr{D}(A) \subseteq \mathscr{D}\left((-A)^{1-\theta}\right)$ holds for $\Re \mathrm{e} \theta \in(1-\beta, \beta)$, which is not our case.

Now that we have given a sense to the purely imaginary powers of $\mathrm{m} .1$. operators $A$ satisfying $(\mathrm{H} 1)$ and $(\mathrm{H} 3)$, future research should investigate if definition (9.4) allows to extend to this class of operators the results mentioned in Sections 2-4 for single-valued operator satisfying (H1) with $\beta=1$, and which require the boundedness of $(-A)^{i t}, t \in \mathbf{R}$. This research is out of the aims of this paper, so that here we limit ourselves to point out two inherent difficulties on it. A first difficult is that we have no explicit representation for $(-A)^{1+i t}$, which is only defined as the inverse of the operator $(-A)^{-(1+i t)} \in \mathscr{L}(X)$ defined by (3.1). This absence of an explicit formula for $(-A)^{1+i t}$ will constitute a problem for examining the boundedness of $(-A)^{i t}$ already in the single-valued case with $(\alpha, \beta) \neq(1,1)$. The second difficult is that in the really multivalued case the boundedness of $(-A)^{i t}$ has to be intended with respect to the norm of $\mathrm{m} .1$. operators. To this purpose, we recall that a $\mathrm{m}$. 1. operator $T$ with domain the whole $X$ is said to be bounded if $\|T\|=\sup _{\|x\|_{X} \leq 1}\left(\inf _{y \in T x}\|y\|_{X}\right)<\infty$. Hence, in general, to prove that a $\mathrm{m}$. 1 . operator is bounded it is not an easy task, at least of not knowing that $T$ has a section $S \in \mathscr{L}(X)$, in which case $\|T\| \leq\|S\|_{\mathscr{L}(X)}$ 
(cf. [6, Proposition II.4.1]). A sufficient condition for the existence of a bounded section $S$ for a given m. 1. operator $T$ is given in [6, Proposition II.4.2]. However, this condition is of difficult application and it requires to a priori know that $T$ is bounded, which is just what we want to prove when $T=(-A)^{i t}$.

\section{References}

[ 1 ] El. H. Alaarabiou: Calcul fonctionnel et puissance fractionarie d'opérateurs linéaires multivoque non négatifs, C. R. Acad. Sci. Paris S6r. I 313 (1991), 163-166 (in French).

[2] H. Amann: On abstract parabolic fundamental solutions, J. Math. Soc. Japan 39 (1987), 93-116.

[ 3 ] H. Amann: Parabolic evolution equations in interpolation and extrapolation spaces, J. Funct. Anal. 78 (1988), 233-270.

[4] A. V. Balakrishnan: Fractional powers of closed operators and the semigroups generated by them, Pacific J. Math. 10 (1960), 419-437.

[ 5] H. Berens, P. L. Butzer: Approximation theorems for semi-group operators in intermediate spaces, Bull. Amer. Math. Soc. 70 (1964), 689-692.

[6] R. Cross: Multivalued Linear Operators, Marcel Dekker Inc., New York-Basel-Hong Kong, 1998.

[ 7 ] G. Dore, A. Venni: On the closedness of the sum of two closed operators, Math. Z. 196 (1987), 189-201.

[ 8 ] A. Favaron: Optimal time and space regularity for solutions of degenerate differential equations, Cent. Eur. J. Math. 7 (2009), 249-271.

[9] A. Favaron, A. Favini: Maximal time regularity for degenerate evolution integro-differential equations, J. Evol. Equ. 10 (2010), 377-412.

[10] A. Favini, A. Yagi: Space and time regularity for degenerate evolution equations, J. Mat. Soc. Japan 44 (1992), 331-350.

[11] A. Favini, A. Yagi: Multivalued linear operators and degenerate evolution equations, Ann. Mat. Pura Appl. (IV) 163 (1993), 353-384.

[12] A. Favini, A. Yagi: Degenerate Differential Equations in Banach Spaces, Marcel Dekker Inc., New York-Basel-Hong Kong, 1999.

[13] A. Favini, A. Yagi: Quasilinear degenerate evolution equations in Banach spaces, J. Evol. Equ. 4 (2004), 421-449.

[14] D. Fujiwara: Concrete characterization of the domains of fractional powers of some elliptic differential operators of the second order, Proc. Japan Acad. 43 (1967), 82-86.

[15] D. Fujiwara: $L^{p}$-theory for characterizing the domain of fractional powers of $-\Delta$ in the half space, J. Fac. Sci. Univ. Tokyo Sec. I, 15 (1968), 169-177.

[16] D. Fujiwara: On the asymptotic behaviour of the Green operators for elliptic boundary problems and the pure imaginary powers of some second order operators, J. Math. Soc. Japan 21 (1969), 480-522.

[17] D. Gilbarg, N. S. Trudinger: Elliptic Partial Differential Equations of Second Order, Reprint of the 1998 Ed., Springer-Verlag Berlin Heidelberg, 2001.

[18] P. Grisvard: Commutativité de deux foncteurs d'interpolation et applications, J. Math. Pures Appl. 45 (1966), 143-290 (in French).

[19] E. Hille, R. S. Phillips: Functional Analysis and Semi-Groups (revised edition), Amer. Math. Soc. Colloq. Publ. 31, Providence, R.I., 1957.

[20] D. Henry: Geometric theory of semilinear parabolic equations, Lecture Notes in Math. 840, Springer-Verlag, New York, 1981.

[21] H. Hoshino, Y. Yamada: Solvability and smoothing effect for semilinear parabolic equations, Funkcial. Ekvac. 34 (1991), 475-494.

[22] H. W. Hövel, U. Westphal: Fractional powers of closed operators, Studia Math. 42 (1977), 177-194. 
[23] H. Komatsu: Fractional powers of operators, Pacific J. Math. 19 (1966), 285-345.

[24] H. Komatsu: Fractional powers of operators, II. Interpolation spaces, Pacific J. Math. 21 (1967), 89-111.

[25] H. Komatsu: Fractional powers of operators, VI. Interpolation of non-negative operators and imbedding theorems, J. Fac. Sci. Univ. Tokyo Sec. I A, Math. 19 (1972), 1-63.

[26] M. A. Krasnosel'skii, P. P. Zabreiko, E. I. Pustylnik, P. E. Sobolevskii: Integral Operators in Spaces of Summable Functions, Noordhoff International Publishing, Leyden, 1976.

[27] A. Lunardi: Analytic semigroups and optimal regularity in parabolic problems, Birkhäuser Verlag, Basel, 1995.

[28] I. V. Mel'nikova: The Cauchy problem for a differential inclusion in Banach spaces and distribution spaces, Siberian Math. J. 42 (2001), 751-765.

[29] F. Periago: Global existence, uniqueness, and continuous dependence for a semilinear initial value problem, J. Math. Anal. Appl. 280 (2003), 413-423.

[30] R. Seeley: Fractional powers of boundary problems, Actes, Congrés. Intern. Math. 1970, Tome 2 (1971), 795-801.

[31] R. Seeley: Norms and domains of complex powers $A_{B}^{z}$, Amer. J. Math. 93 (1971), 299-309.

[32] K. Taira: The theory of semigroups with weak singularities and its application to partial differential equations, Tsukuba J. Math. 13 (1989), 513-562.

[33] H. Tanabe: On the equations of evolution in a Banach space, Osaka J. Math. 12 (1960), $363-$ 376.

[34] H. Tanabe: Functional Analytic Methods for Partial Differential Equations, Marcel Dekker Inc., New York-Basel-Hong Kong, 1997.

[35] H. Triebel: Interpolation Theory, Function Spaces, Differential Operators, North Holland Publ. Co., Amsterdam, 1978.

[36] W. von Wahl: Gebrochene Potenzen eines elliptischen Operators und parabolische Differentialgleichungen in Räumen höldersteiger Funktionen, Nachr. Akad. Wiss. Göttingen Math-Phys. K1. II (1972), 231-258 (in German).

[37] W. von Wahl: Neue Resolventenabschätzungen für elliptische Differentialoperatoren und semilineare parabolische Gleichungen, Abh. Math. Sem. Univ. Hamburg 46 (1977), 179-204 (in German).

[38] C. Wild: Semi-groupes de croissance $\alpha<1$ holomorphes, C. R. Acad. Sci. Paris Sér. A-B 285 (1977), A437-A440 (in French).

[39] A. Yagi: Fractional powers of operators and evolution equations of parabolic type, Proc. Japan Acad. Ser. A Math. Sci. 64 (1988), 227-230.

[40] A. Yagi: Generation theorem of semigroup for multivalued linear operators, Osaka J. Math. 28 (1991), 385-410.

Email: alberto.favaron@fastwebnet.it

Dipartimento di Matematica

Università degli Studi di Bologna

Piazza di Porta San Donato 5, 40126 Bologna

Italy

Email: favini@dm.unibo.it 